Article

\title{
Synthesis and Comparative Structure-Activity Study of Carbohydrate-Based Phenolic Compounds as $\alpha$-Glucosidase Inhibitors and Antioxidants
}

\author{
Shota Machida, Saki Mukai, Rina Kono, Megumi Funato, Hiroaki Saito and Taketo Uchiyama *(D) \\ School of Pharmacy, Nihon University, 7-7-1 Narashinodai, Funabashi, Chiba 274-8555, Japan; \\ phsh18002@g.nihon-u.ac.jp (S.M.); phsa12222@g.nihon-u.ac.jp (S.M.); rinachammm1026ml@icloud.com (R.K.); \\ mgm27.04f@ezweb.ne.jp (M.F.); saito.hiroaki@nihon-u.ac.jp (H.S.) \\ * Correspondence: uchiyama.taketo@nihon-u.ac.jp; Tel.: +81-47-465-6290
}

Received: 7 November 2019; Accepted: 25 November 2019; Published: 27 November 2019

check for updates

\begin{abstract}
Twenty-one natural and unnatural phenolic compounds containing a carbohydrate moiety were synthesized and their structure-activity relationship (SAR) was evaluated for $\alpha$-glucosidase inhibition and antioxidative activity. Varying the position of the galloyl unit on the 1,5-anhydro -D-glucitol (1,5-AG) core resulted in changes in the $\alpha$-glucosidase inhibitory activity and notably, particularly strong activity was demonstrated when the galloyl unit was present at the C-2 position. Furthermore, increasing the number of the galloyl units significantly affected the $\alpha$-glucosidase inhibition, and 2,3,4,6-tetra-galloyl-1,5-AG (54) and 2,3,4,6-tetra-galloyl-D-glucopyranose (61) exhibited excellent activities, which were more than 13 -fold higher than the $\alpha$-glucosidase inhibitory activity of acertannin (37). Moreover, a comparative structure-activity study suggested that a hemiacetal hydroxyl functionality in the carbohydrate core and a biaryl bond of the 4,6-O-hexahydroxydiphenoyl (HHDP) group, which are components of ellagitannins including tellimagrandin I, are not necessary for the $\alpha$-glucosidase inhibitory activity. Lastly, the antioxidant activity increased proportionally with the number of galloyl units.
\end{abstract}

Keywords: 1,5-AG; tellimagrandin I; acertannin; maplexin; ginnalin; polyphenol; $\alpha$-glucosidase; antioxidant

\section{Introduction}

Impaired glucose tolerance increases the risk of vascular events such as atherosclerotic coronary artery disease [1,2]. Particularly, postprandial hyperglycemia is a serious risk factor for cardiovascular diseases and is believed to be the cause of oxidative stress that leads to vascular events [3-7]. Thus, controlling postprandial hyperglycemia is an important target to prevent diabetes as well as diabetic complications. In clinical medicine, $\alpha$-glucosidase inhibitors such as acarbose, miglitol, and voglibose, belong to the class of antidiabetic drugs used for improving postprandial hyperglycemia [8-11]. Currently, natural products and their derivatives constitute more than half of the drugs in the clinic [12-15]. Therefore, finding inspiration in nature to develop more efficient and effective medicines has attracted significant interest.

Trees belonging to the Acer species have been used as traditional medicinal plants for many years and are widely known for their sap, which can be concentrated to produce maple syrup [16]. It has been demonstrated that Acer extracts display various bioactivities such as anti-cancer [17,18], antioxidant [19-22], and antihyperglycemic effects [23,24]. A. Honma et al. identified a compound from Acer saccharum extracts able to suppress hyperglycemia, namely acertannin, and revealed that its effects are a consequence of potent inhibitory activity toward $\alpha$-glucosidase [25]. The structural components of acertannin include the characteristic 1,5-anhydro-D-glucitol (1,5-AG) sugar moiety, 
which lacks the hemiacetal hydroxyl group present in D-glucose, as the carbohydrate core, and two gallic acid functionalities as the phenolic units (Figure 1) [26]. However, only a few plants belonging to the Acer genus produce the 1,5-AG core containing polyphenols $[27,28]$. To date, maplexin A-J and ginnalin $\mathrm{A}-\mathrm{C}$ have been isolated and characterized. The molecules possess varying numbers and positions of the phenol units esterified with the 1,5-AG core [29-32]. These polyphenols were shown to exhibit different bioactivities such as $\alpha$-glucosidase inhibition and antioxidant activity. It is noteworthy that different numbers, positions, and types of the phenol units on the 1,5-AG core display non-identical bioactivities [32-36].

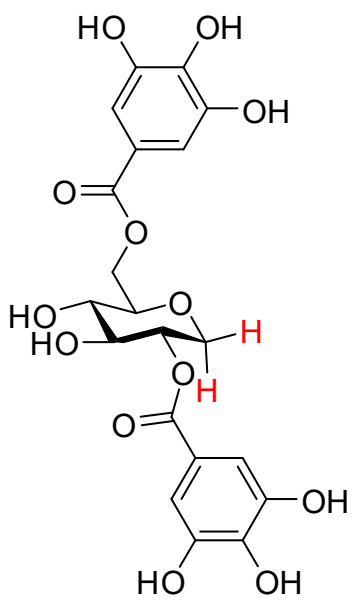

Figure 1. Structure of acertannin.

Tellimagrandin I, which belongs to ellagitannins, has also been demonstrated to be an $\alpha$-glucosidase inhibitor and to show antioxidant activity [37,38]. The molecule is characterized by the presence of a hexahydroxydiphenoyl (HHDP) group, two galloyl units and the D-glucose core possesses a hemiacetal hydroxyl functionality (Figure 2) [39]. The HHDP group provides structural diversity in polyphenols, and the macro-lactone structure is considered to be the element responsible for the pharmacological activity [40]. Nonetheless, to our knowledge, no reports on the evaluation of the synthesis and/or bioactivity of compounds comprising the HHDP functionality on the 1,5-AG core have been reported so far. Furthermore, the hemiacetal hydroxyl group is a fundamental moiety in the carbohydrate chemistry; however, its effects on the bioactivity remain largely unexplored.

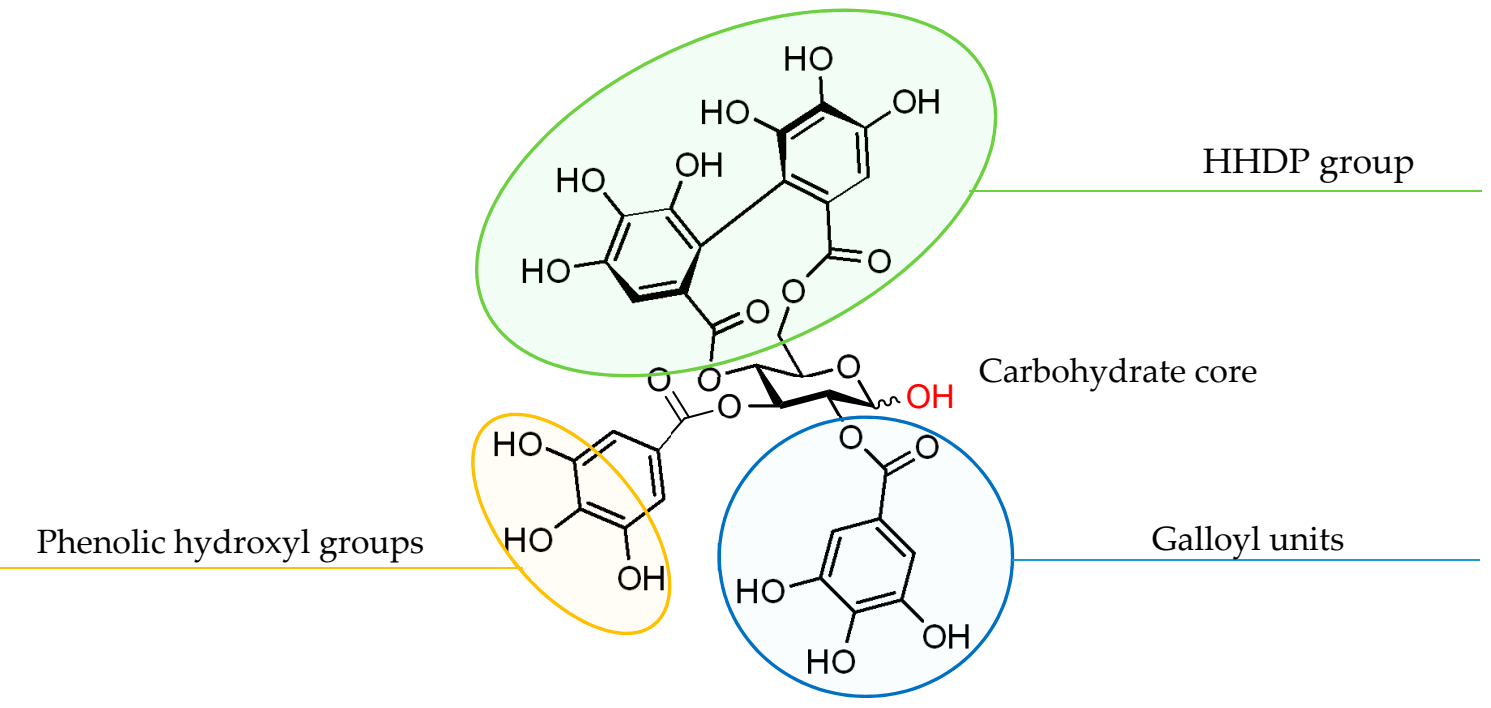

Figure 2. The structural components of tellimagrandin I. HHDP, hexahydroxydiphenoyl. 
In the present study, we report the synthesis of a series of 21 carbohydrate-based phenolic compounds to investigate the structure-activity relationship (SAR). $\alpha$-glucosidase inhibition and antioxidant activity were examined by studying the effects of (1) the position and number of galloyl units, (2) the type of phenol units, (3) the existence the 4,6-O-HHDP group, and (4) the presence of the hemiacetal hydroxyl group.

\section{Results}

\subsection{Syntheses of 1,5-AG-Based Polyphenols}

\subsubsection{Syntheses of Galloylated 1,5-AGs}

Recently, A. Kamori et al. reported the synthesis of various natural and unnatural acertannin derivatives and evaluated their SAR against ceramidase and ceramide synthase enzymes [35]. In addition, we have also previously reported a facile method for the preparation of 1,5-anhydroalditol via treatment of per-O-TMS-glycopyranosyl iodide with $\mathrm{LiBH}_{4}$ [41]. In total, 1,5-AG, which can be easily synthesized from D-glucose on multi-gram scale in three days, possesses four hydroxyl groups; hence, 15 different combinations are possible for mono-, di-, tri-, and tetra-galloylation of 1,5-AG. In the current study, we attempted the synthesis of all of these galloylated compounds starting from 1,5-AG.

Firstly, 4,6-O-benzylidene-1,5-AG (1) [42] was protected with benzyl (Bn) group using BnBr and $\mathrm{NaH}$ to afford di-benzylated compound 2 [42]. However, reacting 1 in a 2-phase dichloromethane (DCM) $/ 5 \% \mathrm{NaOH}$ system with $\mathrm{BnBr}, \mathrm{NaI}$, and tetra- $n$-butylammonium hydrogen sulfate provided the 3-OH analog 3 [35] and the 2-OH analog 4 [35] as a mixture of products, which could be separated by column chromatography (C.C.) (Scheme 1) [43].

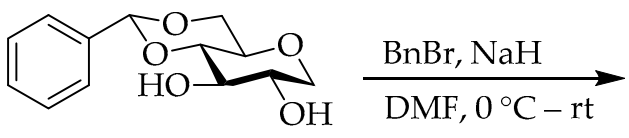

1

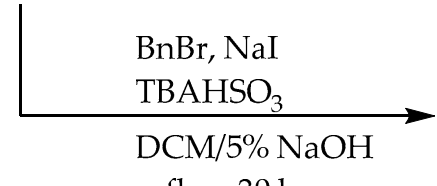

reflux, $30 \mathrm{~h}$

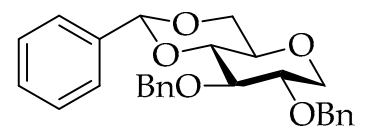

$2(93 \%)$

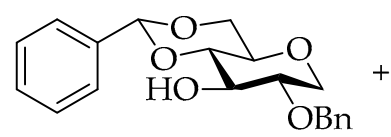

$3(59 \%)$

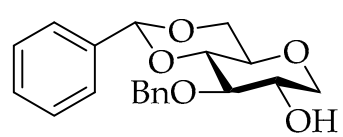

$4(30 \%)$

Scheme 1. Selective protection of 1,5-AG.

Subsequently, the 4,6-O-benzylidene groups on the 1,5-AG derivatives 1-4 were converted to the corresponding hydroxyl moieties using an acid or reducing reagent (Scheme 2). Compounds $\mathbf{2 - 4}$ were then deprotected with $80 \%$ acetic acid/ $\mathrm{H}_{2} \mathrm{O}$ solution to give the corresponding diol analogs 5-7 [35,42]. Reduction of $\mathbf{1}, 3$, and 4 by $\mathrm{BH}_{3} / \mathrm{THF}$ and trimethylsilyl trifluoromethanesulfonate (TMSOTf) [44], and reduction of 2 by diisobutylaluminium hydride (DIBAL-H) [45] resulted in the formation of the 6-OH derivatives 8-11 [35,46,47]. Moreover, compounds 1-4 were treated with triethylsilane and trifluoroacetic acid (TFA) [48] to afford the 4-OH derivatives 12-15 [35,49]. Esterification of these -OH derivatives with Bn-protected gallic acid (16) [50] using $N, N$-dimethyl-4-aminopyridine (DMAP) and triethylamine (TEA), as well as 2-chloro-1-methylpyridinium iodide as the condensing reagent, provided 1,5-AG-based galloylated derivatives 17-30. Finally, hydrogenolysis using $\mathrm{Pd}(\mathrm{OH})_{2}$ as the catalyst in a MeOH/THF solvent mixture gave 14 types of the 1,5-AG core containing polyphenol analogs 31-44 (Scheme 3). 


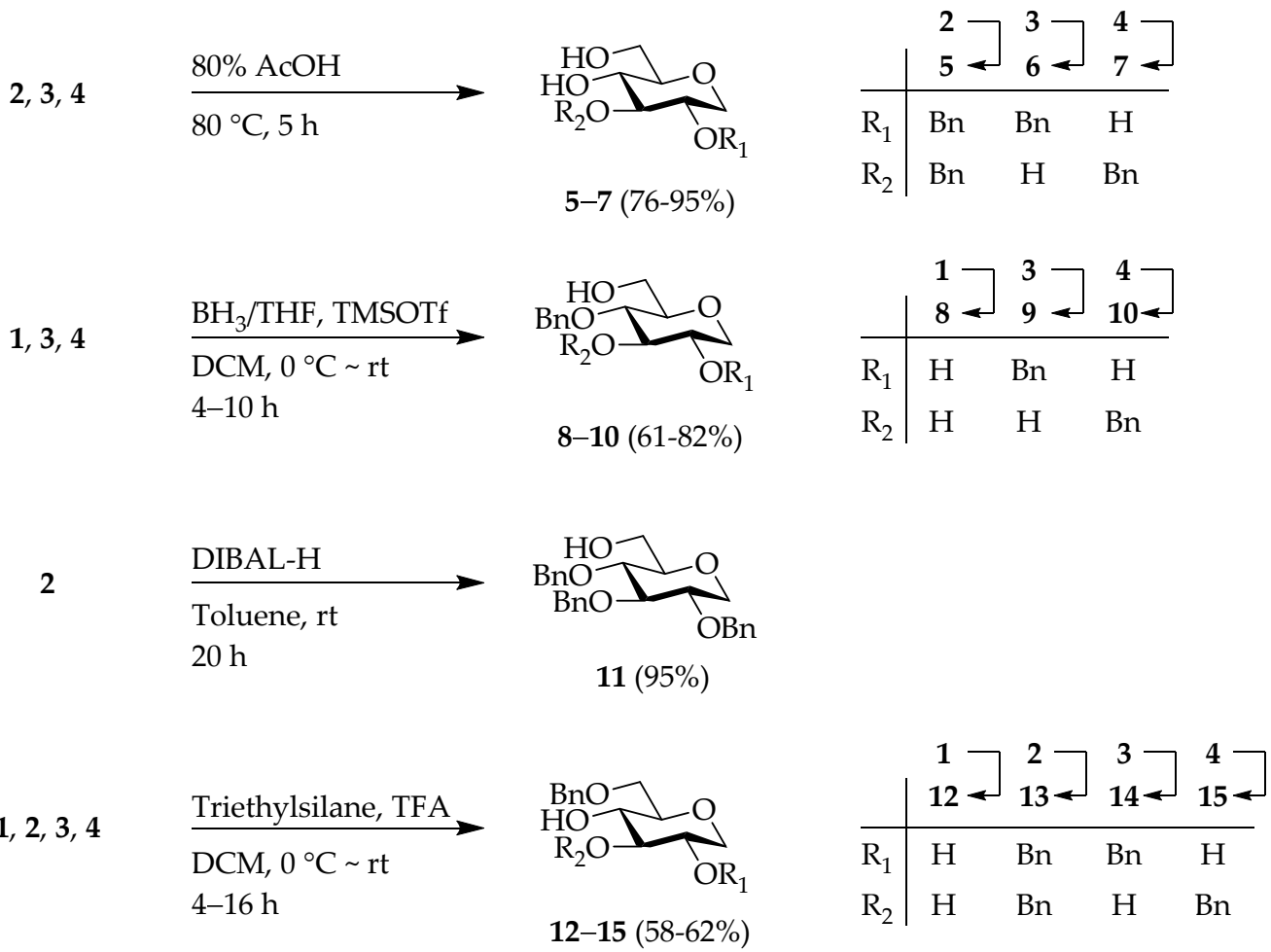

Scheme 2. Deprotection of 1-4.

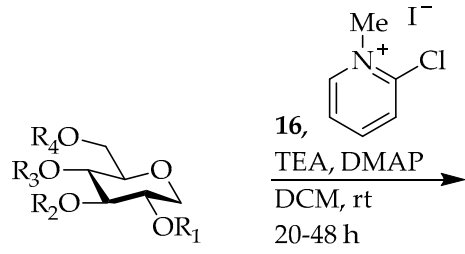

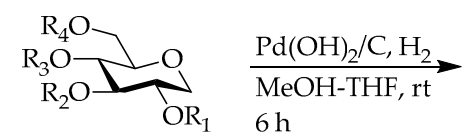

17-30 (41-92\%)

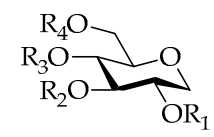

$31-44(95-100 \%)$

\begin{tabular}{c|cccc} 
& $\mathrm{R}_{1}$ & $\mathrm{R}_{2}$ & $\mathrm{R}_{3}$ & $\mathrm{R}_{4}$ \\
\hline $\mathbf{4}$ & $\mathrm{H}$ & $\mathrm{Bn}$ & \multicolumn{2}{c}{ Benzylidene } \\
$\mathbf{3}$ & $\mathrm{Bn}$ & $\mathrm{H}$ & \multicolumn{3}{|c}{ Benzylidene } \\
$\mathbf{1 3}$ & $\mathrm{Bn}$ & $\mathrm{Bn}$ & $\mathrm{H}$ & $\mathrm{Bn}$ \\
$\mathbf{1 1}$ & $\mathrm{Bn}$ & $\mathrm{Bn}$ & $\mathrm{Bn}$ & $\mathrm{H}$ \\
$\mathbf{1}$ & $\mathrm{H}$ & $\mathrm{H}$ & Benzylidene \\
$\mathbf{1 5}$ & $\mathrm{H}$ & $\mathrm{Bn}$ & $\mathrm{H}$ & $\mathrm{Bn}$ \\
$\mathbf{1 0}$ & $\mathrm{H}$ & $\mathrm{Bn}$ & $\mathrm{Bn}$ & $\mathrm{H}$ \\
$\mathbf{1 4}$ & $\mathrm{Bn}$ & $\mathrm{H}$ & $\mathrm{H}$ & $\mathrm{Bn}$ \\
$\mathbf{9}$ & $\mathrm{Bn}$ & $\mathrm{H}$ & $\mathrm{Bn}$ & $\mathrm{H}$ \\
$\mathbf{5}$ & $\mathrm{Bn}$ & $\mathrm{Bn}$ & $\mathrm{H}$ & $\mathrm{H}$ \\
$\mathbf{1 2}$ & $\mathrm{H}$ & $\mathrm{H}$ & $\mathrm{H}$ & $\mathrm{Bn}$ \\
$\mathbf{8}$ & $\mathrm{H}$ & $\mathrm{H}$ & $\mathrm{Bn}$ & $\mathrm{H}$ \\
$\mathbf{7}$ & $\mathrm{H}$ & $\mathrm{Bn}$ & $\mathrm{H}$ & $\mathrm{H}$ \\
$\mathbf{6}$ & $\mathrm{Bn}$ & $\mathrm{H}$ & $\mathrm{H}$ & $\mathrm{H}$
\end{tabular}

\begin{tabular}{c|cccc} 
& $R_{1}$ & $R_{2}$ & $R_{3}$ & $R_{4}$ \\
\hline $\mathbf{1 7}$ & $B n G$ & $B n$ & Benzylidene \\
$\mathbf{1 8}$ & $\mathrm{Bn}$ & $\mathrm{BnG}$ & Benzylidene \\
$\mathbf{1 9}$ & $\mathrm{Bn}$ & $\mathrm{Bn}$ & $\mathrm{BnG}$ & $\mathrm{Bn}$ \\
$\mathbf{2 0}$ & $\mathrm{Bn}$ & $\mathrm{Bn}$ & $\mathrm{Bn}$ & $\mathrm{BnG}$ \\
$\mathbf{2 1}$ & $\mathrm{BnG}$ & $\mathrm{BnG}$ & $\mathrm{Benzylidene}$ \\
$\mathbf{2 2}$ & $\mathrm{BnG}$ & $\mathrm{Bn}$ & $\mathrm{BnG}$ & $\mathrm{Bn}$ \\
$\mathbf{2 3}$ & $\mathrm{BnG}$ & $\mathrm{Bn}$ & $\mathrm{Bn}$ & $\mathrm{BnG}$ \\
$\mathbf{2 4}$ & $\mathrm{Bn}$ & $\mathrm{BnG}$ & $\mathrm{BnG}$ & $\mathrm{Bn}$ \\
$\mathbf{2 5}$ & $\mathrm{Bn}$ & $\mathrm{BnG}$ & $\mathrm{Bn}$ & $\mathrm{BnG}$ \\
$\mathbf{2 6}$ & $\mathrm{Bn}$ & $\mathrm{Bn}$ & $\mathrm{BnG}$ & $\mathrm{BnG}$ \\
$\mathbf{2 7}$ & $\mathrm{BnG}$ & $\mathrm{BnG}$ & $\mathrm{BnG}$ & $\mathrm{Bn}$ \\
$\mathbf{2 8}$ & $\mathrm{BnG}$ & $\mathrm{BnG}$ & $\mathrm{Bn}$ & $\mathrm{BnG}$ \\
$\mathbf{2 9}$ & $\mathrm{BnG}$ & $\mathrm{Bn}$ & $\mathrm{BnG}$ & $\mathrm{BnG}$ \\
$\mathbf{3 0}$ & $\mathrm{Bn}$ & $\mathrm{BnG}$ & $\mathrm{BnG}$ & $\mathrm{BnG}$
\end{tabular}

\begin{tabular}{l|cccc} 
& $R_{1}$ & $R_{2}$ & $R_{3}$ & $R_{4}$ \\
\hline 31 & $G$ & $H$ & $H$ & $H$ \\
32 & $H$ & $G$ & $H$ & $H$ \\
33 & $H$ & $H$ & $G$ & $H$ \\
34 & $H$ & $H$ & $H$ & $G$ \\
35 & $G$ & $G$ & $H$ & $H$ \\
36 & $G$ & $H$ & $G$ & $H$ \\
37 & $G$ & $H$ & $H$ & $G$ \\
38 & $H$ & $G$ & $G$ & $H$ \\
39 & $H$ & $G$ & $H$ & $G$ \\
40 & $H$ & $H$ & $G$ & $G$ \\
41 & $G$ & $G$ & $G$ & $H$ \\
42 & $G$ & $G$ & $H$ & $G$ \\
43 & $G$ & $H$ & $G$ & $G$ \\
44 & $H$ & $G$ & $G$ & $G$
\end{tabular}<smiles>O=C(O)c1cc(Br)c(OCc2ccccc2)c(OCc2ccccc2)c1</smiles><smiles>CC(=O)c1cc(OCc2ccccc2)c(OCc2ccccc2)c(OCc2ccccc2)c1</smiles><smiles>O=C(I)c1cc(O)c(O)c(O)c1</smiles>

Scheme 3. Synthesis of galloylated 1,5-AGs. 


\subsubsection{Synthesis of Maplexin J Analogs}

Maplexin J (54) [32,50], which is per-galloylated 1,5-AG, could be easily synthesized from 1,5-AG. Therefore, we attempted to synthesize of maplexin J analogs, which in addition to the galloyl moiety, contained different numbers of phenolic groups at various positions intending to elucidate the effect of this functionality on the bioactivity. Phenol derivatives 45-48 [51-53] were prepared in 2-steps from commercially available methyl esters (Scheme 4). Subsequently, 1,5-AG was condensed with 16 [50], and 45-48 using DMAP, TEA, and 2-chloro-methylpyridinium iodide to obtain the corresponding Bn-protected 1,5-AG analogs 49-53. Deprotection under hydrogenolysis conditions provided compounds 54-58 (Scheme 5). However, esterification of benzyl glucoside 59 [54] with 16 using 1-ethyl-3-(3-dimethylaminopropyl)carbodiimide (EDC) and DMAP resulted in the formation of intermediate 60 . Following hydrogenolysis, tetra-galloylated D-glucopyranose 61 containing a hemiacetal moiety was formed. Notably, the structure of 61 [55] is analogous to tellimagrandin I; however, the biaryl bond of the HHDP group is missing (Scheme 6).<smiles>[M]OC(=O)c1cc([R])c([R2])c([R])c1</smiles>

1) $\mathrm{BnCl}, \mathrm{NaI}, \mathrm{K}_{2} \mathrm{CO}_{3}$

$\underset{\text { 2) } \mathrm{KOH}, \mathrm{MeOH} / 1,4 \text {-Dioxane }}{\stackrel{\text { Acetone, reflux, } 8 \mathrm{~h}}{\longrightarrow}}$ reflux, $2 \mathrm{~h}$<smiles>[R]c1cc(C(=O)O)cc([R3])c1[R]</smiles>

\begin{tabular}{c|ccc} 
& $\mathrm{R}_{1}$ & $\mathrm{R}_{2}$ & $\mathrm{R}_{3}$ \\
\hline 45 & $\mathrm{OBn}$ & $\mathrm{OBn}$ & $\mathrm{H}$ \\
46 & $\mathrm{OBn}$ & $\mathrm{H}$ & $\mathrm{OBn}$ \\
47 & $\mathrm{OBn}$ & $\mathrm{H}$ & $\mathrm{H}$ \\
48 & $\mathrm{H}$ & $\mathrm{OBn}$ & $\mathrm{H}$
\end{tabular}

45-48 (60-98\%, 2 steps)

Scheme 4. Preparation of the Bn-protected phenol units 45-48.

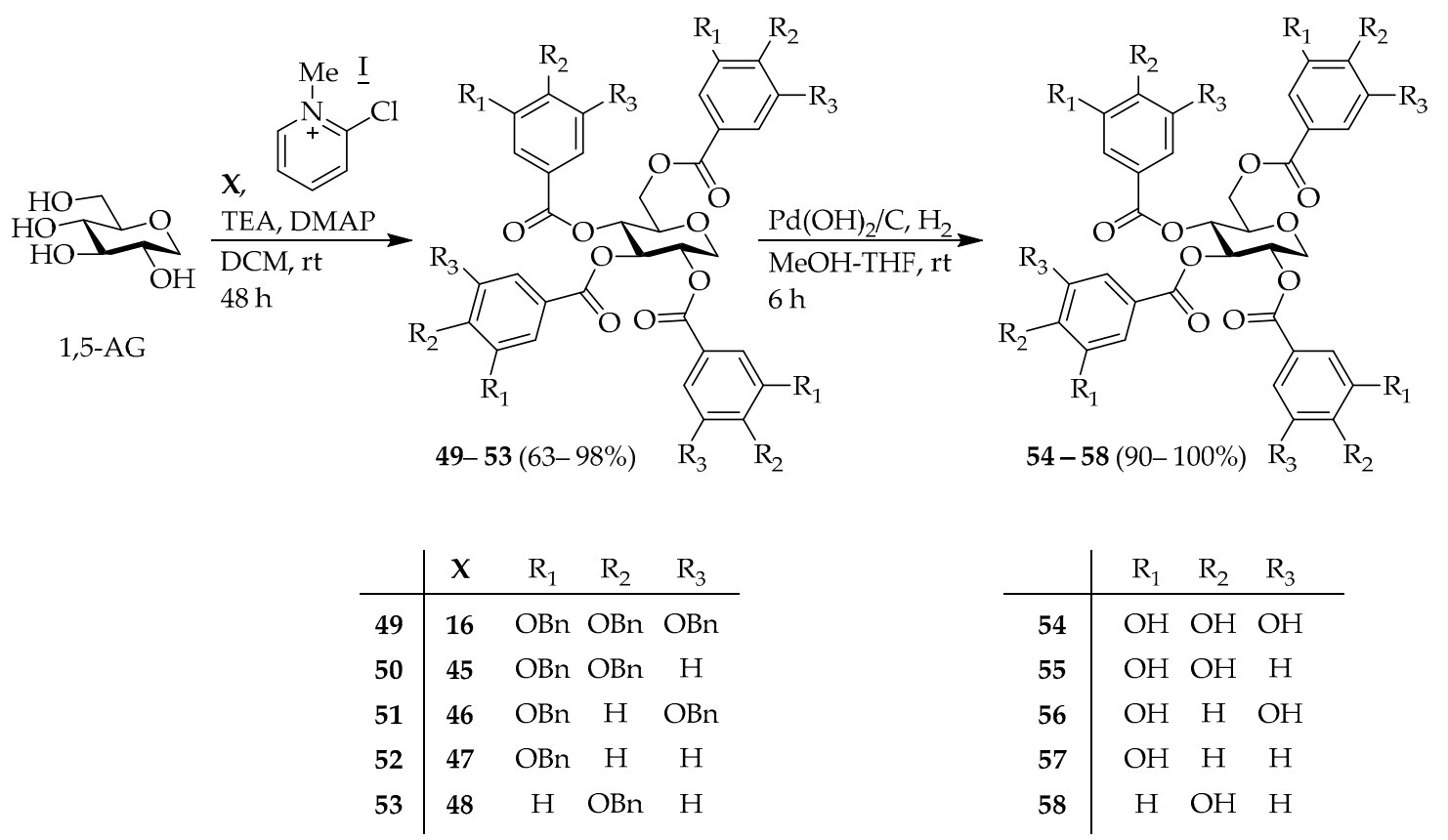

Scheme 5. Synthesis of maplexin J analogs 54-58. 


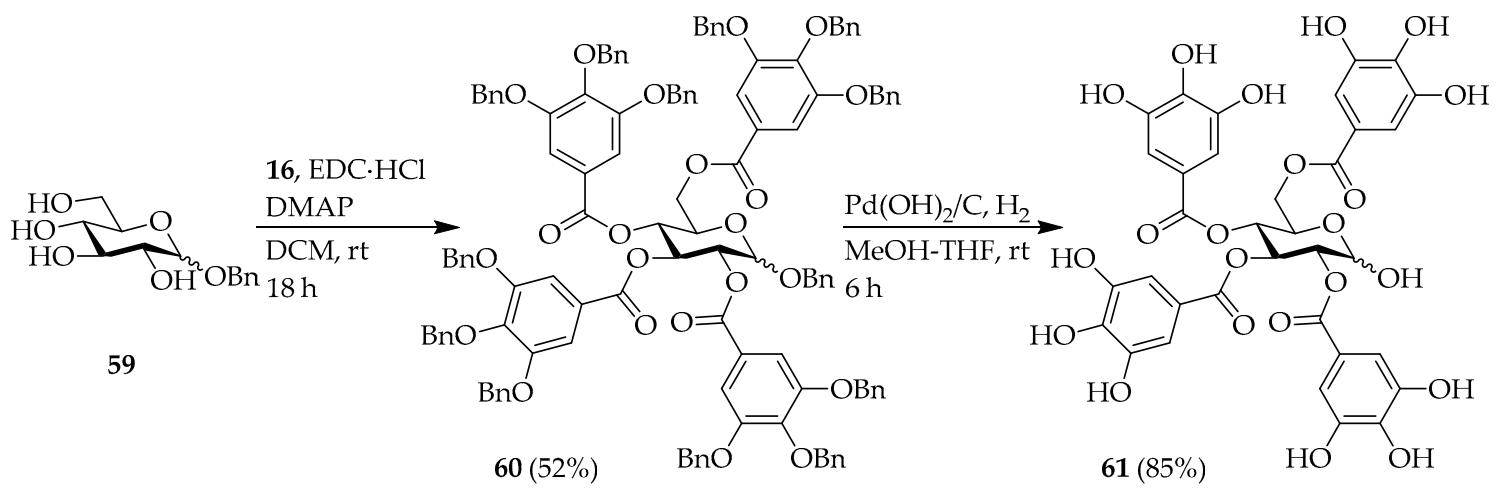

Scheme 6. Synthesis of tellimagrandin I analog 61 .

\subsubsection{Synthesis of a 1,5-AG-Based Tellimagrandin I Analog}

To consider the effect of the hemiacetal group on the activity of tellimagrandin I, we also focused on the synthesis of the 1-deoxy analog 67. Deprotection of benzylidene acetal in $\mathbf{2 1}$ in a methanol/DCM solvent system using iodine, according to the method reported by Feldman et al. [56], gave diol 62 in a high yield of $93 \%$ (Scheme 7). Intermediate 62 was then condensed with the gallic acid derivative 63 [57] to afford the galloylated compound 64. The subsequent removal of the methoxymethyl (MOM) protecting groups provided 65 in $93 \%$ yield.
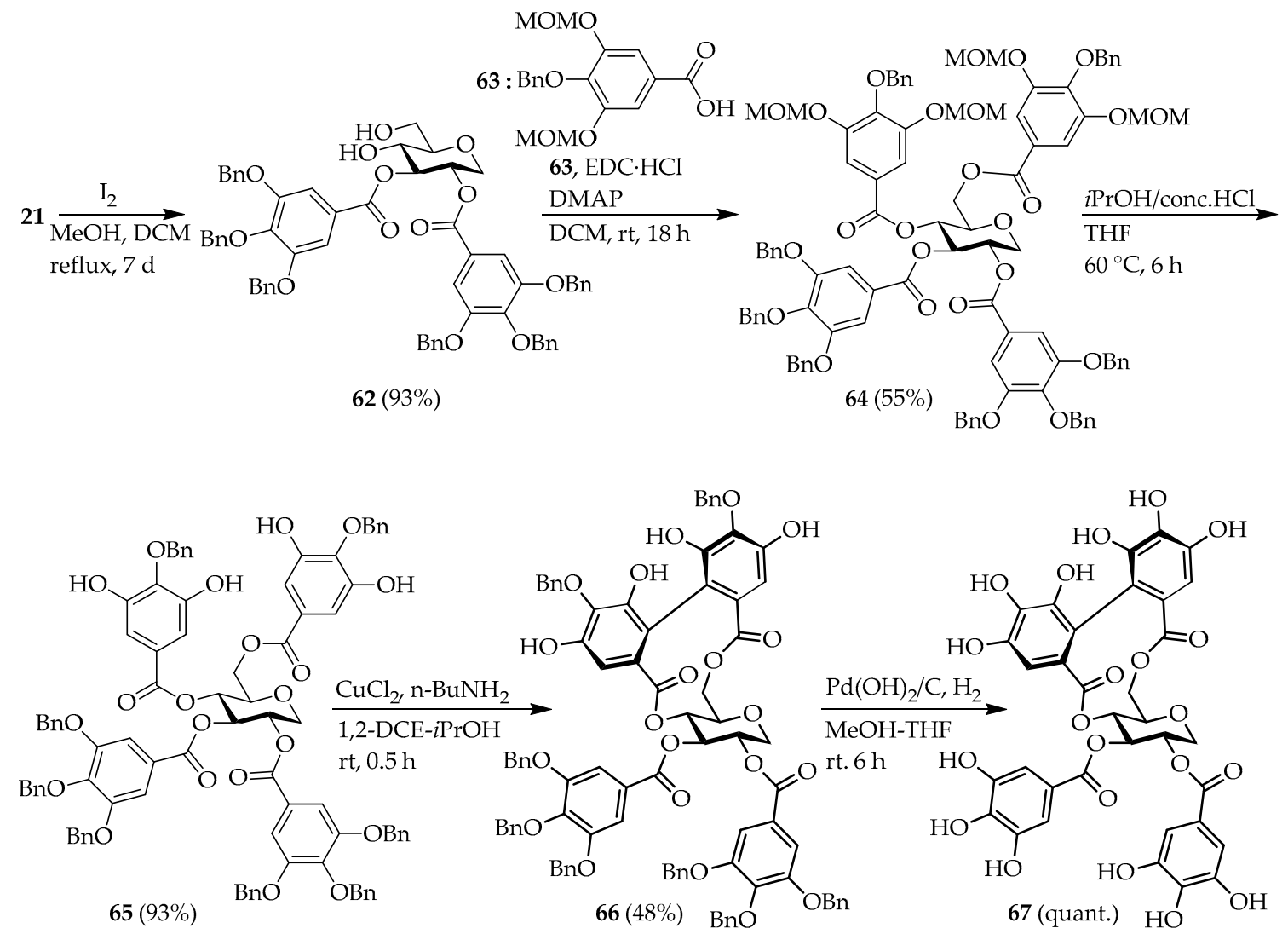

Scheme 7. Synthesis of the 1,5-AG-based tellimagrandin I analog 67.

The construction of the HHDP group was performed in accordance with the approach previously reported by Yamada et al. [57-60]. Compound 65 was treated with $n-\mathrm{BuNH}_{2}$ and $\mathrm{CuCl}_{2}$ to provide the biaryl derivative 66 . Finally, hydrogenation of 66 in $\mathrm{MeOH} / \mathrm{THF}$ with $\mathrm{Pd}(\mathrm{OH})_{2}$ as the catalyst gave the desired 1,5-AG-based tellimagrandin I analog 67. 


\subsection{Evaluation of $\alpha$-Glucosidase Inhibition and Antioxidant Activity}

\subsubsection{The $\alpha$-Glucosidase Inhibitory Activity}

The $\alpha$-glucosidase inhibitory activity of all samples was assayed utilizing a commercially available FUJIFILM $\alpha$-glucosidase inhibitory activity assay kit. In total, $25 \mu \mathrm{L}$ of each sample $(25-2000 \mu \mathrm{g} / \mathrm{mL}$ in $\left.\mathrm{H}_{2} \mathrm{O}\right)$ or acarbose $\left(0.5-8 \mu \mathrm{g} / \mathrm{mL}\right.$ in $\left.\mathrm{H}_{2} \mathrm{O}\right), 50 \mu \mathrm{L}$ of $18.5 \mathrm{mM}$ maltose diluted in maleic anhydride buffer $(100 \mathrm{mM}, \mathrm{pH}=6.0)$, and $25 \mu \mathrm{L}$ of the rat $\alpha$-glucosidase solution were incubated in a micro-tube at $37^{\circ} \mathrm{C}$ for $30 \mathrm{~min}$. Subsequently, $400 \mu \mathrm{L}$ of purified water was added to the solution and the reaction mixture was boiled for $3 \mathrm{~min}$ to deactivate $\alpha$-glucosidase. The generated glucose was measured by LabAssay ${ }^{\mathrm{TM}}$ glucose (mutarotase-GOD method). In total, $100 \mu \mathrm{L}$ of the reaction solution and $150 \mu \mathrm{L}$ of the coloring solution were incubated in 96 -well plates at $37^{\circ} \mathrm{C}$ for $10 \mathrm{~min}$, and the absorbance was recorded at $505 \mathrm{~nm}$ using a microplate reader (Bio-Rad, Model 680). The inhibition percentage was calculated using the following Equation:

$$
\text { Inhibition percentage }(\%)=1-\frac{\mathrm{As}-\mathrm{Ab}}{\mathrm{Ac}} \times 100
$$

where As is the absorbance of the analyzed sample, $\mathrm{Ab}$ is the absorbance of the blank (immediate deactivation), and Ac is the absorbance of the control (without $\alpha$-glucosidase). Acarbose was used as the positive control.

\subsubsection{Evaluation of Antioxidant Activity}

The antioxidant activity was assessed by employing the 2,2-diphenyl-1-picrylhydrazyl (DPPH) radical scavenging assay, according to the previously published method [61]. In total, $100 \mu \mathrm{L}$ of each sample $\left(1000-10 \mu \mathrm{g} / \mathrm{mL}\right.$ in $\left.50 \% \mathrm{EtOH} / \mathrm{H}_{2} \mathrm{O}\right)$ or trolox $\left(160-0 \mu \mathrm{M}\right.$ in $\left.50 \% \mathrm{EtOH} / \mathrm{H}_{2} \mathrm{O}\right)$, and $50 \mu \mathrm{L}$ of 2-(N-morpholino)ethanesulfonic acid (MES)-NaOH buffer $(200 \mathrm{mM}, \mathrm{pH}=6.0)$, and $50 \mu \mathrm{L}$ of an $800 \mu \mathrm{M}$ DPPH diluted in $99.5 \%$ EtOH were mixed in 96-well plates. Following shaking the microplate for $15 \mathrm{~min}$ in the dark, the absorbance was recorded at $520 \mathrm{~nm}$ using a microplate reader (Bio-Rad, Model 680). Effective percentages (\%) were calculated as follows:

$$
\text { Effective percentages }(\%)=1-\frac{\mathrm{As}}{\mathrm{Ab}} \times 100
$$

where As is the absorbance of the sample and $\mathrm{Ab}$ is the absorbance of the blank (without samples, $50 \% \mathrm{EtOH} / \mathrm{H}_{2} \mathrm{O}$ solution was used instead).

In addition, correlation of the absorbance on the $y$-axis and the concentrations on the $x$-axis resulted in the formation of an approximately straight-line plot. The trolox-equivalent (sample-mol/trolox-mol) was calculated as follows:

$$
\text { Trolox - equivalent }\left(\frac{\mathrm{mol}-\text { trolox }}{\mathrm{mol}-\text { sample }}\right)=\frac{\mathrm{Ss}}{\mathrm{St}}
$$

where Ss is the slope of the sample and St is the slope of trolox.

\section{Discussion}

The results of the biological evaluation of the 21 synthesized compounds considering the $\alpha$-glucosidase inhibitory and antioxidant activities are summarized in Table 1. 
Table 1. $\alpha$-Glucosidase inhibitory and antioxidant activities.

\begin{tabular}{|c|c|c|c|}
\hline \multirow{2}{*}{ Compounds } & \multirow{2}{*}{$\begin{array}{c}\alpha \text {-Glucosidase } \\
\mathrm{IC}_{50}(\mu \mathrm{M})^{a}\end{array}$} & \multicolumn{2}{|c|}{ Antioxidant } \\
\hline & & $\mathrm{EC}_{50}(\mu \mathrm{M})^{b}$ & Trolox-eq $^{b}$ \\
\hline \multicolumn{4}{|l|}{ Mono- } \\
\hline 2-galloyl (Ginnalin C) [31] 31 & $95.1 \pm 0.22$ & $19.40 \pm 1.02$ & $2.04 \pm 0.08$ \\
\hline 3-galloyl (Maplexin A) [29] 32 & $137.9 \pm 1.50$ & $20.50 \pm 2.05$ & $2.38 \pm 0.24$ \\
\hline 4-galloyl (Maplexin B) [29] 33 & $143.9 \pm 0.52$ & $20.80 \pm 1.54$ & $2.09 \pm 0.19$ \\
\hline 6-galloyl (Ginnalin B) [31] 34 & $127.1 \pm 0.88$ & $22.90 \pm 1.61$ & $2.13 \pm 0.12$ \\
\hline \multicolumn{4}{|l|}{ Di- } \\
\hline 2,3-galloyl (Maplexin C) [29] 35 & $20.50 \pm 0.50$ & $11.30 \pm 0.98$ & $4.29 \pm 0.23$ \\
\hline 2,4-galloyl (Maplexin D) [29] 36 & $48.30 \pm 1.25$ & $14.30 \pm 0.29$ & $3.36 \pm 0.13$ \\
\hline 2,6-galloyl (Acertannin) [25] 37 & $35.60 \pm 2.58$ & $15.20 \pm 0.53$ & $3.32 \pm 0.37$ \\
\hline 3,4-galloyl $38^{c}$ & $91.40 \pm 6.56$ & $14.80 \pm 0.56$ & $3.55 \pm 0.42$ \\
\hline 3,6-galloyl 39 & $55.00 \pm 8.84$ & $11.90 \pm 0.71$ & $3.67 \pm 0.19$ \\
\hline 4,6-galloyl 40 & $26.60 \pm 2.37$ & $13.00 \pm 1.12$ & $3.78 \pm 0.33$ \\
\hline \multicolumn{4}{|l|}{ Tri- } \\
\hline 2,3,4-galloyl 41 & $6.72 \pm 0.21$ & $7.94 \pm 0.39$ & $5.59 \pm 0.26$ \\
\hline 2,3,6-galloyl (Maplexin F) [30] 42 & $5.34 \pm 0.55$ & $8.09 \pm 0.41$ & $5.75 \pm 0.18$ \\
\hline 2,4,6-galloyl (Maplexin E) [29] 43 & $9.34 \pm 0.99$ & $10.30 \pm 0.76$ & $4.31 \pm 0.35$ \\
\hline 3,4,6-galloyl 44 & $12.60 \pm 0.61$ & $8.48 \pm 0.21$ & $5.36 \pm 0.20$ \\
\hline \multicolumn{4}{|l|}{ Tetra- } \\
\hline 2,3,4,6-galloyl (Maplexin J) [32] 54 & $2.56 \pm 0.10$ & $6.61 \pm 0.68$ & $6.77 \pm 0.63$ \\
\hline $3^{\prime}, 4^{\prime}$-dihydroxybenzoyl $55^{c}$ & $3.28 \pm 0.16$ & $6.99 \pm 0.11$ & $6.54 \pm 0.15$ \\
\hline $3^{\prime}, 5^{\prime}$-dihydroxybenzoyl $56^{c}$ & $9.34 \pm 0.02$ & $70<$ & n.d $e^{e}$ \\
\hline $3^{\prime}$-hydroxybenzoyl $57^{c}$ & $-^{d}$ & $78<$ & n.d ${ }^{e}$ \\
\hline $4^{\prime}$-hydroxybenzoyl $58^{c}$ & $-{ }^{d}$ & $78<$ & n.d ${ }^{e}$ \\
\hline 1-OH-2,3,4,6-galloyl 61 & $1.68 \pm 0.21$ & $5.60 \pm 0.28$ & $8.33 \pm 0.49$ \\
\hline 2,3-galloyl-4,6-HHDP $67^{c}$ & $3.22 \pm 0.51$ & $6.79 \pm 0.21$ & $6.44 \pm 0.24$ \\
\hline Tellimagrandin I & $3.37 \pm 0.04$ & $6.31 \pm 0.27$ & $7.04 \pm 0.37$ \\
\hline Methyl gallate & $90.50 \pm 6.97$ & $19.60 \pm 0.11$ & $2.42 \pm 0.27$ \\
\hline Methyl 3,4-dihydroxybenzoate & $300<<$ & $21.10 \pm 1.08$ & $2.20 \pm 0.05$ \\
\hline Methyl 3,5-dihydroxybenzoate & $300<<$ & n.d ${ }^{e}$ & n.d $e^{e}$ \\
\hline Methyl 3-hydroxybenzoate & $660<<$ & n.d ${ }^{e}$ & n.d ${ }^{e}$ \\
\hline Methyl 4-hydroxybenzoate & $660<<$ & n.d ${ }^{e}$ & n.d ${ }^{e}$ \\
\hline Acarbose & $0.11 \pm 0.02$ & - & - \\
\hline Trolox & - & $49.40 \pm 3.87$ & - \\
\hline
\end{tabular}

${ }^{a} \mathrm{IC}_{50}$ data represents mean \pm S.D. of $\mathrm{n}=2 .{ }^{b} \mathrm{EC}_{50}$ and trolox-eq data represents mean \pm S.D. of $\mathrm{n}=3 .{ }^{c}$ Novel compound. ${ }^{d}$ Did not dissolve in water. ${ }^{e}$ n.d.: not detected.

The comparison of the 1,5-AG-based polyphenol analogs 31-44 and 54 revealed that the $\alpha$-glucosidase inhibitory activity significantly increased with the number of the galloyl units in the compounds and the highest inhibition was observed for tetra-O-galloyl-1,5-AG (maplexin J) $54\left(\mathrm{IC}_{50}=2.56 \mu \mathrm{M}\right)$. This result is in accordance with the previous reports [32]. In addition, different the position of the galloyl unit on the 1,5-AG core appeared to influence the $\alpha$-glucosidase inhibitory activity, even for the compounds with the same number of these moieties. Analogous outcomes were noted for 2-galloyl-1,5-AG (31) and methyl gallate $\left(\mathrm{IC}_{50}=95.1,90.5 \mu \mathrm{M}\right.$, respectively). Conversely, differing $\alpha$-glucosidase inhibitory activities were obtained for mono-galloyl analogs 32-34, which are weaker inhibitors than methyl gallate $\left(\mathrm{IC}_{50}=127.1-143.9 \mu \mathrm{M}\right)$. Furthermore, compound 33 (galloyl unit at the C-4 position) exhibited lowest activity $\left(\mathrm{IC}_{50}=143.9 \mu \mathrm{M}\right)$. Higher inhibitory activity was detected for di-galloylated analogs 35-37 $\left(\mathrm{IC}_{50}=20.5-48.3 \mu \mathrm{M}\right)$, which possessed the galloyl unit at the C-2 position than for analogs 38-40 $\left(\mathrm{IC}_{50}=26.6-91.4 \mu \mathrm{M}\right)$. In particular, compound 35 that contains esterified galloyl units at the C-2 and C-3 positions exhibited three-times higher inhibitory activity than gallic acid. Moreover, analog 38, galloylated at the C-3 and C-4 positions, displayed significantly lower activity $\left(\mathrm{IC}_{50}=91.4\right)$. Among the tri-galloyllated analogs, compound 42 without a galloyl unit at the C-4 position showed stronger inhibitory activity $\left(\mathrm{IC}_{50}=5.34 \mu \mathrm{M}\right)$ than analogs 41,43 , and $44\left(\mathrm{IC}_{50}=6.72,9.34\right.$, and $12.6 \mu \mathrm{M}$, 
respectively). In addition, analog 44 without a galloyl moiety at the C-2 position displayed weak activity. Thus, our results suggested that a galloyl unit at the C-2 position considerably increases the $\alpha$-glucosidase inhibitory activity, while the presence of this group at the C-4 position causes a decrease in the activity.

Subsequently, we compared maplexin J (54) and its analogs (55 and 56) to elucidate the effect of the phenolic hydroxyl group. The $3^{\prime}, 4^{\prime}$-di-hydroxybenzoyl analog 55 exhibited good inhibitory activity, whereas the $3^{\prime}, 5^{\prime}$-di-hydroxybenzoyl analog 56 was a weaker inhibitor of the $\alpha$-glucosidase enzyme $\left(\mathrm{IC}_{50}=3.28,9.34 \mu \mathrm{M}\right.$, respectively). Consequently, these results implied that the presence of two adjacent phenolic hydroxyl groups is essential for the desired activity. We then focused on the evaluation of the influence of the hemiacetal hydroxyl and the HHDP functionalities against the $\alpha$-glucosidase inhibitory activity. The effect of the hemiacetal hydroxyl moiety can be observed by the comparison of the activity of maplexin $\mathrm{J}(54)$ and its analog 61 ( $\mathrm{IC}_{50}=1.68,2.56 \mu \mathrm{M}$, respectively). Furthermore, tellimagrandin I and 67 showed analogous inhibitory activity $\left(\mathrm{IC}_{50}=3.37,3.22 \mu \mathrm{M}\right.$, respectively). Therefore, the obtained outcomes suggested that the presence of a hemiacetal hydroxyl group did not have a significant effect on the $\alpha$-glucosidase inhibitory activity. Lastly, to examine the influence of the HHDP group, the results for maplexin J (54) and the 4,6-O-HHDP analog 67 were compared and it transpired that maplexin J (54) displayed marginally higher activity than its analog $67\left(\mathrm{IC}_{50}=2.56,3.22 \mu \mathrm{M}\right.$, respectively). Likewise, the assessment of the tellimagrandin I activity and the activity of analog $\mathbf{6 1}$ without the HHDP group revealed that $\mathbf{6 1}$ was a stronger inhibitor than tellimagrandin $\mathrm{I}\left(\mathrm{IC}_{50}=3.37,1.68 \mu \mathrm{M}\right.$, respectively). Intriguingly, our results suggested that the 4,6-O-HHDP group has a weakening effect on the $\alpha$-glucosidase inhibitory activity [38,62].

Meanwhile, we also investigated the antioxidant activity of these polyphenols and their analogs. Firstly, the mono-galloylated analogs 31-34 showed nearly equivalent antioxidative activity with methyl gallate $\left(\mathrm{EC}_{50}=19.4-22.9 \mu \mathrm{M}, \mathrm{TE}=2.04-2.42\right)$. Moreover, the activity improved as the number of galloyl units increased, with di-galloylated analogs $\left(\mathrm{EC}_{50}=11.9-15.2 \mu \mathrm{M}, \mathrm{TE}=3.32-4.29\right)$ exhibiting lower activity than the tri-galloylated analogs $\left(\mathrm{EC}_{50}=7.94-10.3 \mu \mathrm{M}, \mathrm{TE}=4.31-5.75\right)$ and the tetra-galloylated analogs 54, 61 displaying the highest activity $\left(\mathrm{EC}_{50}=5.60,6.61 \mu \mathrm{M}, \mathrm{TE}=8.33,6.77\right.$, respectively). Unlike the significant increase observed for the $\alpha$-glucosidase inhibitory activity, the antioxidant activity increased proportionally to the number of galloyl units. Moreover, the antioxidant activity was not affected by the position of the galloyl units on the carbohydrate core. In addition, the comparison of compounds 54, 61, and 67 with tellimagrandin I revealed similar activity $\left(\mathrm{EC}_{50}=6.61,5.60,6.79\right.$, and $6.31 \mu \mathrm{M}, \mathrm{TE}=6.77,8.33,6.44$, and 7.04, respectively). This implied that the antioxidative activity was not influenced by the presence of a hemiacetal hydroxyl and the 4,6-O-HHDP groups. The presence of the phenolic hydroxyl groups in 3,4-dihydroxybenzoyl analogs 55 and methyl 3,4-dihydroxybenzoate appeared to result in improved antioxidative activity $\left(\mathrm{EC}_{50}=6.99 \mu \mathrm{M}\right.$, $\mathrm{TE}=6.54$ ); however, no antioxidant activity was observed for 3,5-dihydroxybenzoyl analog 56 and the monohydroxybenzoyl analogs (57 and 58). Likewise, the activity of methyl 3,4-dihydroxybenzoate was comparable with methyl gallate $\left(\mathrm{EC}_{50}=21.1 \mu \mathrm{M}, \mathrm{TE}=2.20\right)$, whereas methyl 3,5-dihydroxybenzoate, methyl 3- and 4-hydroxybenzoate did not exhibit any notable activity. This data therefore suggested that the presence of two adjacent phenolic hydroxyl groups is necessary for the antioxidant activity, as was the case with $\alpha$-glucosidase inhibitory activity.

\section{Conclusions}

We synthesized 21 carbohydrate-based phenolic analogs including a series of compounds containing all possible combinations of galloylation on 1,5-AG. The $\alpha$-glucosidase inhibition and antioxidant activities of these compounds were further studied to evaluate the SAR. Our results suggested that the $\alpha$-glucosidase inhibitory activity; 1 ) is significantly enhanced with the increasing number of galloyl units, and changing the position of the galloyl moiety substitution on the 1,5-AG unit tends to affect the activity; particularly, the presence of this functionality at the C-2 position improves the $\alpha$-glucosidase inhibition, whereas substitution at the $C-4$ position reduces it, 2 ) requires 
two adjacent phenolic hydroxyl groups, 3 ) is not affected by the presence of the biaryl bond on the 4,6-O-HHDP group , 4) is not influenced by the hemiacetal hydroxyl functionality on the carbohydrate unit. Moreover, the following trends were determined for the antioxidant activity; 1 ) the activity is dependent on the number of galloyl units; however, it is not affected by their position, 2) the presence of two adjacent phenolic hydroxyl groups is significant, 3 ) the activity is not affected by the HHDP group or the hemiacetal hydroxyl group. The $\alpha$-glucosidase inhibitory activity is undoubtedly affected by the position of the galloyl group on 1,5-AG. This outcome indicates that the carbohydrate core is not only a store unit for the galloyl moiety but can also act as a carrier to the biological targets. Thus, derivatives modified at the C-2 or C-4 positions on the carbohydrate unit, which is not D-glucose, have the potential to exhibit stronger antidiabetic activity. The synthesis and profiling of further analogs will be reported in due course.

\section{Materials and Methods}

\subsection{General Information}

Proton nuclear magnetic resonance $\left({ }^{1} \mathrm{H}\right.$ NMR) spectra were recorded on JEOL JNM-ECX600 spectrometers. Chemical shifts are reported relative to internal standard (tetramethylsilane; $\delta_{\mathrm{H}} 0.00$, $\left.\mathrm{CDCl}_{3} ; \delta_{\mathrm{H}} 7.26\right)$. Data are presented as follows: chemical shift $(\delta, \mathrm{ppm})$, multiplicity $(\mathrm{s}=$ singlet, $\mathrm{d}=$ doublet, $\mathrm{t}=$ triplet, $\mathrm{q}=$ quartet, $\mathrm{m}=$ multiplet), coupling constant and integration. Carbon nuclear magnetic resonance $\left({ }^{13} \mathrm{C}\right.$ NMR) spectra were recorded on JEOL JNM-ECX600 $(150 \mathrm{MHz})$ spectrometers. The following internal reference was used: (tetramethylsilane: $\delta 0.00, \mathrm{CDCl}_{3} \delta 77.0$, Acetone $-d_{6}$; $\left.\delta 29.8, \mathrm{CD}_{3} \mathrm{OD} ; \delta 49.0\right)$. Optical rotations were measured on a JASCO P-1030 digital polarimeter at the sodium D line ( $589 \mathrm{~nm})$. Electron impact (EI) mass analyses and fast atom bombardment (FAB) mass analyses were carried out with a JEOL JMS-GCMATE. C.C. was carried out on Kanto Silica gel 60N spherical (63-210 mesh). Analytical thin-layer chromatography (TLC) was carried out using Merck Kieselgel 60 F254 plates with visualization by ultraviolet light or stained by $8 \% \mathrm{H}_{2} \mathrm{SO}_{4} / \mathrm{EtOH}$ solution on hot-plate. Tellimagrandin I was purchased from Nagara Science (Gifu, Japan). Methyl gallate, methyl 3,4-dihydroxybenzoate, methyl 3,5-dihydroxybenzoate, methyl 3-hydroxybenzoate, methyl 4-hydroxybenzoate, Trolox and MES were purchased from TOKYO CHEMICAL INDUSTRY CO., LTD (Tokyo, Japan). DPPH was purchased from Sigma-Aldrich Japan (Tokyo, Japan).

\subsection{Chemical Synthesis}

2-O-Benzyl-4,6-O-benzylidene-1,5-anhydro-D-glucitol (3) [35] and 3-O-Benzyl-4,6-O-benzylidene-1,5 -anhydro-D-glucitol (4) [35]; Compound $1(2.5 \mathrm{~g}, 10 \mathrm{mmol})$ and TBAHSO $_{4}(0.68 \mathrm{~g}, 2.0 \mathrm{mmol})$ in $160 \mathrm{~mL}$ of DCM and $14 \mathrm{~mL}$ of $5 \% \mathrm{NaOH}$ was stirred at rt. Then, $\mathrm{BnBr}(2.1 \mathrm{~mL}, 17 \mathrm{mmol})$ was slowly added, and the mixture was refluxed for $30 \mathrm{~h}$. After the addition $50 \mathrm{~mL}$ of water, the mixture was extracted with $\mathrm{DCM}(3 \times 100)$. The combined organic layers were washed with brine, dried over $\mathrm{Na}_{2} \mathrm{SO}_{4}$, filtered and concentrated under reduced pressure. The crude product was separated by C.C $(\mathrm{Hex} / \mathrm{EtOAc}=4 / 1)$ to obtain 2-O-Bn compound $3(2.0 \mathrm{~g}, 59 \%)$ as colorless needles and 3-O-Bn compound $4(1.0 \mathrm{~g}, 30 \%$ yield $)$ as a white solid. Compound 3: m.p. $163^{\circ} \mathrm{C} ;[\alpha]_{\mathrm{D}}^{20}=-3.16\left(\mathrm{c} 1.0, \mathrm{CHCl}_{3}\right) ;{ }^{1} \mathrm{H} \mathrm{NMR}\left(\mathrm{CDCl}_{3}, 600 \mathrm{MHz}\right) \delta$ 7.49-7.47 (m, 2H), 7.38-7.29 (m, 8H), 5.50 (s, 1H), 4.76, $4.67(\mathrm{ABq}, J=11.7 \mathrm{~Hz}, 2 \mathrm{H}), 4.30(\mathrm{dd}, J=10.5$, $5.0 \mathrm{~Hz}, 1 \mathrm{H}), 4.01(\mathrm{dd}, J=11.3,5.5 \mathrm{~Hz}, 1 \mathrm{H}), 3.84(\mathrm{~m}, 1 \mathrm{H}), 3.65(\mathrm{t}, J=10.3 \mathrm{~Hz}, 1 \mathrm{H}), 3.59-3.55(\mathrm{~m}, 1 \mathrm{H})$, $3.45(\mathrm{t}, J=9.3 \mathrm{~Hz}, 1 \mathrm{H}), 3.35(\mathrm{~m}, 1 \mathrm{H}), 3.30(\mathrm{t}, J=11.0 \mathrm{~Hz}, 1 \mathrm{H}) ;{ }^{13} \mathrm{C} \mathrm{NMR}\left(\mathrm{CDCl}_{3}, 150 \mathrm{MHz}\right) \delta 138.02$, 137.05, 129.23, 128.56, 128.33, 128.03, 127.89, 126.29, 101.88, 81.05, 77.74, 74.81, 73.43, 70.91, 68.79, 68.45. Compound 4: m.p. $137^{\circ} \mathrm{C} ;[\alpha]_{\mathrm{D}}^{20}=+5.3\left(\mathrm{c} 1.0, \mathrm{CHCl}_{3}\right) ;{ }^{1} \mathrm{H} \mathrm{NMR}\left(\mathrm{CDCl}_{3}, 600 \mathrm{MHz}\right) \delta 7.50-7.48(\mathrm{~m}, 2 \mathrm{H})$, $7.41-7.29(\mathrm{~m}, 8 \mathrm{H}), 5.58(\mathrm{~s}, 1 \mathrm{H}), 5.03,4.72(\mathrm{ABq}, J=11.3 \mathrm{~Hz}, 2 \mathrm{H}), 4.34(\mathrm{dd}, J=10.5,5.0 \mathrm{~Hz}, 1 \mathrm{H}), 4.06$ $(\mathrm{dd}, J=11.2,5.7 \mathrm{~Hz}, 1 \mathrm{H}), 3.80-3.76(\mathrm{~m}, 1 \mathrm{H}), 3.72(\mathrm{t}, J=10.3 \mathrm{~Hz}, 1 \mathrm{H}), 3.66(\mathrm{t}, J=9.1 \mathrm{~Hz}, 1 \mathrm{H}), 3.58$ $(\mathrm{t}, J=8.8 \mathrm{~Hz}, 1 \mathrm{H}), 3.44-3.40(\mathrm{~m}, 1 \mathrm{H}), 3.34(\mathrm{t}, J=10.8 \mathrm{~Hz}, 1 \mathrm{H}) ;{ }^{13} \mathrm{C} \mathrm{NMR}\left(\mathrm{CDCl}_{3}, 150 \mathrm{MHz}\right) \delta 138.30$, $137.32,129.00,128.61,128.30,128.13,128.01,125.98,101.21,82.66,82.13,74.71,71.53,69.91,69.79,68.88$. 
2,3-Di-O-benzyl-1,5-anhydro-D-glucitol (5) [42]; Compound 2 (0.86g, $2.0 \mathrm{mmol})$ in $10 \mathrm{~mL}$ of $80 \%$ $\mathrm{AcOH} / \mathrm{H}_{2} \mathrm{O}$ solution was stirred at $80^{\circ} \mathrm{C}$ for $5 \mathrm{~h}$. After the addition $5 \mathrm{~mL}$ of saturated aq. $\mathrm{NaHCO}_{3}$, the reaction solution was extracted by EtOAc $(3 \times 30)$. The organic layer was washed with brine, dried over $\mathrm{Na}_{2} \mathrm{SO}_{4}$, filtered and concentrated under reduced pressure. The crude was purified by recrystallization (EtOAc/Hex) to obtain $5\left(0.65\right.$ g, 95\%) as colorless needles. m.p. $129^{\circ} \mathrm{C} ;[\alpha]_{\mathrm{D}}^{20}=-10.4$ (c 1.00, $\left.\mathrm{CHCl}_{3}\right) ;{ }^{1} \mathrm{H}$ NMR $\left(\mathrm{CDCl}_{3}, 600 \mathrm{MHz}\right) \delta 7.43-7.29(\mathrm{~m}, 10 \mathrm{H}), 5.03,4.70(\mathrm{ABq}, J=11.7 \mathrm{~Hz}, 2 \mathrm{H})$, 4.64, $4.03(\mathrm{ABq}, J=11.3,2 \mathrm{H}), 3.86-3.82(\mathrm{~m}, 1 \mathrm{H}), 3.72-3.68(\mathrm{~m}, 1 \mathrm{H}), 3.63-3.59(\mathrm{~m}, 1 \mathrm{H}), 3.50-3.44(\mathrm{~m}, 2 \mathrm{H})$, 3.28-3.23 (m, 2H); ${ }^{13} \mathrm{C}$ NMR $\left(\mathrm{CDCl}_{3}, 150 \mathrm{MHz}\right) \delta 138.54,137.97,128.67,128.53,127.98,127.93,127.83$, $85.30,79.53,78.29,75.10,73.07,70.45,67.94,62.89$.

1,5-Anhydro-2-O-benzyl-D-glucitol (6) [35]; Compound 3 (860 mg, $2.5 \mathrm{mmol}$ ) in $10 \mathrm{~mL}$ of $80 \%$ $\mathrm{AcOH} / \mathrm{H}_{2} \mathrm{O}$ solution was stirred at $80^{\circ} \mathrm{C}$ for $5 \mathrm{~h}$. By the same procedure previously described for the preparation of compound 5 , compound $6(503 \mathrm{mg}, 76 \%)$ was obtained as colorless needles. m.p. $131{ }^{\circ} \mathrm{C}$; $[\alpha]_{\mathrm{D}}^{20}=+8.4(\mathrm{c} 1.00, \mathrm{MeOH}) ;{ }^{1} \mathrm{H}$ NMR $\left(\mathrm{CD}_{3} \mathrm{OD}, 600 \mathrm{MHz}\right) ; \delta 7.40-7.36(\mathrm{~m}, 2 \mathrm{H}), 7.33-7.31(\mathrm{~m}, 2 \mathrm{H})$, $7.28-7.25(\mathrm{~m}, 1 \mathrm{H}), 4.75,4.64(\mathrm{ABq}, J=11.7 \mathrm{~Hz}, 2 \mathrm{H}), 3.96(\mathrm{dd}, J=11.0,5.2 \mathrm{~Hz}, 1 \mathrm{H}), 3.81(\mathrm{dd}, J=11.9$, $2.2 \mathrm{~Hz}, 1 \mathrm{H}), 3.59(\mathrm{dd}, J=11.9,6.0 \mathrm{~Hz}, 1 \mathrm{H}), 3.43(\mathrm{t}, J=8.9 \mathrm{~Hz}, 1 \mathrm{H}), 3.39-3.35(\mathrm{~m}, 1 \mathrm{H}), 3.26-3.22(\mathrm{~m}, 1 \mathrm{H})$, 3.15-3.11 (m, 2H); ${ }^{13} \mathrm{C}$ NMR (CD $\left.{ }_{3} \mathrm{OD}, 150 \mathrm{MHz}\right) ; \delta 140.09,129.37,129.05,128.76,82.42,79.31,79.27$, 74.14, 71.92, 68.95, 63.07.

1,5-Anhydro-3-O-benzyl-D-glucitol (7); Compound 4 ( $680 \mathrm{mg}, 2.0 \mathrm{mmol}$ ) in $10 \mathrm{~mL}$ of $80 \% \mathrm{AcOH} / \mathrm{H}_{2} \mathrm{O}$ solution was stirred at $80^{\circ} \mathrm{C}$ for $5 \mathrm{~h}$. By the same procedure previously described for the preparation of compound 5 , compound $7(410 \mathrm{mg}, 80 \%)$ was obtained as colorless needles. m.p. $153^{\circ} \mathrm{C} ;[\alpha]_{\mathrm{D}}^{20}=+28.6$ (c 1.00, MeOH); ${ }^{1} \mathrm{H}$ NMR $\left(\mathrm{CD}_{3} \mathrm{OD}, 600 \mathrm{MHz}\right) ; \delta 7.44(\mathrm{~m}, 2 \mathrm{H}), 7.32-7.30(\mathrm{~m}, 2 \mathrm{H}), 7.26-7.23(\mathrm{~m}, 1 \mathrm{H})$, 4.90 (overlap, 2H), $3.89(\mathrm{dd}, J=11.3,5.5 \mathrm{~Hz}, 1 \mathrm{H}), 3.83(\mathrm{dd}, J=11.9,2.2 \mathrm{~Hz}, 1 \mathrm{H}), 3.62-3.57(\mathrm{~m}, 2 \mathrm{H}), 3.37$ $(\mathrm{dd}, J=18.0,8.71 \mathrm{~Hz}, 1 \mathrm{H}), 3.31-3.28$ (overlap, $1 \mathrm{H}), 3.22-3.16(\mathrm{~m}, 2 \mathrm{H}) ;{ }^{13} \mathrm{C}$ NMR $\left(\mathrm{CD}_{3} \mathrm{OD}, 150 \mathrm{MHz}\right)$; $\delta 140.53,129.20,129.09,128.49,88.20,82.68,76.10,71.67,71.54,71.09,63.05$. HRMS (ESI, $m / z):[\mathrm{M}+\mathrm{Na}]^{+}$, calcd for $\left[\mathrm{C}_{13} \mathrm{H}_{18} \mathrm{O}_{5} \mathrm{Na}\right]^{+}$: 277.1052; found 277.1052.

1,5-Anhydro-4-O-benzyl-D-glucitol (8) [35]; Compound $\mathbf{1}(0.76 \mathrm{~g}, 3.0 \mathrm{mmol})$ in $15 \mathrm{~mL}$ of DCM was stirred at $0{ }^{\circ} \mathrm{C}$. Then, $15 \mathrm{~mL}$ of borane-THF (ca. $1 \mathrm{M}$ THF solution) and TMSOTf $(0.11 \mathrm{~mL}, 0.60 \mathrm{mmol})$ were successively added to the mixture. The mixture was allowed to stir for $4 \mathrm{~h}$ and added $\mathrm{MeOH}$ carefully. After the addition $1 \mathrm{~mL}$ of saturated aq. $\mathrm{NaHCO}_{3}$, the reaction solution was extracted with DCM $(5 \times 40 \mathrm{~mL})$. The combined organic layer was dried over $\mathrm{Na}_{2} \mathrm{SO}_{4}$, filtered and concentrated under reduced pressure. The crude product was purified by C.C. $\left(\mathrm{CHCl}_{3} / \mathrm{MeOH}=8 / 1\right)$ to obtain 8 $(0.64 \mathrm{~g}, 74 \%)$ as a white solid. $[\alpha]_{\mathrm{D}}^{20}=+27.7\left(\mathrm{c} 0.45, \mathrm{CHCl}_{3}\right) ;{ }^{1} \mathrm{H} \mathrm{NMR}\left(\mathrm{CD}_{3} \mathrm{OD}, 600 \mathrm{MHz}\right) \delta 7.38-7.25$ $(\mathrm{m}, 5 \mathrm{H}), 4.94,4.64(\mathrm{ABq}, J=11.0 \mathrm{~Hz}, 2 \mathrm{H}), 3.89(\mathrm{dd}, J=13.3,5.4 \mathrm{~Hz}, 1 \mathrm{H}), 3.78(\mathrm{dd}, J=12.0,2.1 \mathrm{~Hz}, 1 \mathrm{H})$, $3.60(\mathrm{dd}, 1 \mathrm{H}, J=11.4,5.73 \mathrm{~Hz}$ ), 3.49-3.46 (m, 2H), 3.33-3.30 (overlap, $1 \mathrm{H}), 3.20$ (ddd, $J=9.7,5.2,2.1 \mathrm{~Hz}$, $1 \mathrm{H}), 3.14(\mathrm{t}, J=10.7,1 \mathrm{H}) ;{ }^{13} \mathrm{C}$ NMR $\left(\mathrm{CD}_{3} \mathrm{OD}, 150 \mathrm{MHz}\right) ; \delta 140.09,129.33,129.14,128.70,81.75,80.43$, 79.50, 75.90, 71.77, 70.93, 62.72 .

2,4-Di-O-benzyl-1,5-anhydro-D-glucitol (9) [46]; Compound 3 (1.0 g, $3.0 \mathrm{mmol}$ ) in $15 \mathrm{~mL}$ of DCM was successively added $15 \mathrm{~mL}$ of borane-THF (ca. 1M THF solution) and TMSOTf $(0.11 \mathrm{~mL}, 0.6 \mathrm{mmol})$ at $0{ }^{\circ} \mathrm{C}$. The mixture was allowed to stir at $\mathrm{rt}$ for $10 \mathrm{~h}$ and added $1 \mathrm{~mL}$ of $\mathrm{MeOH}$ carefully. After the addition $1 \mathrm{~mL}$ of saturated aq. $\mathrm{NaHCO}_{3}$, the mixture was extracted with $\mathrm{DCM}(3 \times 40 \mathrm{~mL})$. The organic layer was washed with brine, dried over $\mathrm{Na}_{2} \mathrm{SO}_{4}$, filtered and concentrated under reduced pressure. The crude was purified by recrystallization (Hex/EtOAc) to obtain $9(820 \mathrm{mg}, 82 \%)$ as colorless needles. m.p. $112{ }^{\circ} \mathrm{C} ;[\alpha]_{\mathrm{D}}^{20}=+34.6\left(\mathrm{c} 1.00, \mathrm{CHCl}_{3}\right) ;{ }^{1} \mathrm{H} \mathrm{NMR}\left(\mathrm{CDCl}_{3}, 600 \mathrm{MHz}\right) \delta 7.37-7.28(\mathrm{~m}, 10 \mathrm{H}), 4.86,4.70$ $(\mathrm{ABq}, J=11.3 \mathrm{~Hz}, 2 \mathrm{H}), 4.65(\mathrm{~s}, 2 \mathrm{H}), 3.99(\mathrm{dd}, J=11.3,5.2 \mathrm{~Hz}, 1 \mathrm{H}), 3.84(\mathrm{ddd}, J=11.8,5.8,2.7 \mathrm{~Hz}, 1 \mathrm{H})$, $3.74(\mathrm{td}, J=8.9,2.1 \mathrm{~Hz}, 1 \mathrm{H}), 3.68-3.64(\mathrm{~m}, 1 \mathrm{H}), 3.45-3.41(\mathrm{~m}, 1 \mathrm{H}), 3.41(\mathrm{t}, J=9.2 \mathrm{~Hz}, 1 \mathrm{H}), 3.26(\mathrm{ddd}$, $J=9.6,4.5,2.7 \mathrm{~Hz}, 1 \mathrm{H}), 3.19(\mathrm{t}, J=10.8 \mathrm{~Hz}, 1 \mathrm{H}) ;{ }^{13} \mathrm{C} \mathrm{NMR}\left(\mathrm{CDCl}_{3}, 150 \mathrm{MHz}\right) \delta 138.18,138.00,128.60$, $128.56,128.09,127.98,127.85,79.34,78.18,77.97,77.39,74.74,73.05,67.44,62.27$.

3,4-Di-O-benzyl-1,5-anhydro-D-glucitol (10) [47]; Compound 4 ( $850 \mathrm{~g}, 2.5 \mathrm{mmol})$ in $15 \mathrm{~mL}$ of DCM was successively added $12.5 \mathrm{~mL}$ of borane-THF (ca. 1M THF solution) and TMSOTf (90 $\mu \mathrm{L}, 0.5 \mathrm{mmol}$ ) at $0{ }^{\circ} \mathrm{C}$. The mixture was allowed to stir at $\mathrm{rt}$ for $7 \mathrm{~h}$ and added $5 \mathrm{~mL}$ of MeOH carefully. After the 
addition $1 \mathrm{~mL}$ of saturated aq. $\mathrm{NaHCO}_{3}$, the mixture was extracted with $\mathrm{DCM}(3 \times 40 \mathrm{~mL})$. The organic layer was washed with brine, dried over $\mathrm{Na}_{2} \mathrm{SO}_{4}$, filtered and concentrated under reduced pressure. The crude was purified by recrystallization (Hex/EtOAc) to obtain 10 (523 $\mathrm{mg}, 61 \%)$ as colorless needles. m.p. $99{ }^{\circ} \mathrm{C} ;[\alpha]_{\mathrm{D}}^{20}=+48.3\left(\mathrm{c} 1.40, \mathrm{CHCl}_{3}\right) ;{ }^{1} \mathrm{H} \mathrm{NMR}\left(\mathrm{CDCl}_{3}, 600 \mathrm{MHz}\right) \delta 7.38-7.30(\mathrm{~m}, 10 \mathrm{H}), 4.97,4.77$ $(\mathrm{ABq}, J=11.3 \mathrm{~Hz}, 2 \mathrm{H}), 4.86,4.68(\mathrm{ABq}, J=11.0 \mathrm{~Hz}, 2 \mathrm{H}), 3.98(\mathrm{dd}, J=11.2,5.3 \mathrm{~Hz}, 1 \mathrm{H}), 3.84(\mathrm{ddd}$, $J=11.9,5.5,2.6 \mathrm{~Hz}, 1 \mathrm{H}), 3.71-3.65(\mathrm{~m}, 2 \mathrm{H}), 3.52(\mathrm{t}, J=9.1 \mathrm{~Hz}, 1 \mathrm{H}), 3.47(\mathrm{t}, J=8.8 \mathrm{~Hz}, 1 \mathrm{H}), 3.32-3.29$ $(\mathrm{m}, 1 \mathrm{H}), 3.23(\mathrm{t}, J=10.8 \mathrm{~Hz}, 1 \mathrm{H}) ;{ }^{13} \mathrm{C} \mathrm{NMR}\left(\mathrm{CDCl}_{3}, 150 \mathrm{MHz}\right) \delta 138.45,137.84,128.71,128.56,128.02$, $127.86,86.73,79.92,77.79,75.25,74.95,70.16,69.30,61.99$.

2,3,4-Tri-O-benzyl-1,5-anhydro-D-glucitol (11) [46]; Compound 2 (860 mg, $2.0 \mathrm{mmol}$ ) in $10 \mathrm{~mL}$ of toluene was stirred at $\mathrm{rt}$. The reaction solution was added DIBAL-H (ca. 1M toluene solution, $6 \mathrm{~mL}$ ) and stirred at $\mathrm{rt}$ for $20 \mathrm{~h}$. The reaction solution was slowly added $4.2 \mathrm{~mL}$ of $\mathrm{MeOH}$ and $7.2 \mathrm{~mL}$ of $30 \%$ Rochelle salt solution and stirred for $1 \mathrm{~h}$. After the addition $20 \mathrm{~mL}$ of EtOAc, the mixture was extracted with $30 \%$ Rochelle salt solution $(3 \times 15 \mathrm{~mL})$. The organic layer was washed with brine, dried over $\mathrm{Na}_{2} \mathrm{SO}_{4}$, filtered and concentrated under reduced pressure. The crude was purified by C.C. $(\mathrm{Hex} / \mathrm{EtOAc}=4 / 1-2 / 1)$ to obtain $11(760 \mathrm{mg}, 90 \%$ yield $)$ as colorless needles. m.p. $83{ }^{\circ} \mathrm{C} ;[\alpha]_{\mathrm{D}}^{20}=+8.9$ (c 0.90, $\left.\mathrm{CHCl}_{3}\right) ;{ }^{1} \mathrm{H}$ NMR $\left(\mathrm{CDCl}_{3}, 600 \mathrm{MHz}\right) \delta 7.37-7.28(\mathrm{~m}, 15 \mathrm{H}), 4.98-4.64(\mathrm{~m}, 6 \mathrm{H}), 3.99(\mathrm{dd}, \mathrm{J}=11.3$, $5.2 \mathrm{~Hz}, 1 \mathrm{H}), 3.84-3.81(\mathrm{~m}, 1 \mathrm{H}), 3.67-3.58(\mathrm{~m}, 3 \mathrm{H}), 3.48(\mathrm{t}, J=9.3 \mathrm{~Hz}, 1 \mathrm{H}), 3.29-3.25(\mathrm{~m}, 1 \mathrm{H}), 3.23$ $(\mathrm{t}, J=10.8 \mathrm{~Hz}, 1 \mathrm{H}) ;{ }^{13} \mathrm{C} \mathrm{NMR}\left(\mathrm{CDCl}_{3}, 150 \mathrm{MHz}\right) \delta 138.61,138.09,138.01,128.48,128.41,128.05,127.89$, $127.83,127.64,86.17,79.69,78.55,77.57,75.55,75.13,73.34,67.96,62.25$.

1,5-Anhydro-6-O-benzyl-D-glucitol (12) [35]; Compound 1 (760 mg, $3.0 \mathrm{mmol})$ in $15 \mathrm{~mL}$ of DCM was added triethylsilane $(2.4 \mathrm{~mL}, 15 \mathrm{mmol})$ and trifluoracetic acid $(1.2 \mathrm{~mL}, 15 \mathrm{mmol})$ at $0{ }^{\circ} \mathrm{C}$. The reaction solution was stirred at $\mathrm{rt}$ for $8 \mathrm{~h}$. After the addition $10 \mathrm{~mL}$ of saturated aq. $\mathrm{NaHCO}_{3}$, the mixture was extracted with DCM $(5 \times 30 \mathrm{~mL})$. The combined organic layer was dried over $\mathrm{Na}_{2} \mathrm{SO}_{4}$, filtered and concentrated under reduced pressure. The crude was purified by C.C $\left(\mathrm{CHCl}_{3} / \mathrm{MeOH}=100 / 1-10 / 1\right)$ to obtain $12(471 \mathrm{mg}, 62 \%)$ as a colorless oil. $[\alpha]_{\mathrm{D}}^{20}=+8.9\left(\mathrm{c} 0.30, \mathrm{CHCl}_{3}\right) ;{ }^{1} \mathrm{H} \mathrm{NMR}\left(\mathrm{CDCl}_{3}, 600 \mathrm{MHz}\right)$ $\delta 7.35-7.25(\mathrm{~m}, 5 \mathrm{H}), 4.55(\mathrm{~d}, J=2.1 \mathrm{~Hz}, 2 \mathrm{H}), 3.88(\mathrm{dd}, J=11.0,5.5 \mathrm{~Hz}, 1 \mathrm{H}), 3.77(\mathrm{dd}, J=10.8,1.9 \mathrm{~Hz}$, $1 \mathrm{H}), 3.60(\mathrm{dd}, J=10.8,5.7 \mathrm{~Hz}, 1 \mathrm{H}), 3.48-3.42(\mathrm{~m}, 1 \mathrm{H}), 3.32-3.24$ (overlap, $3 \mathrm{H}), 3.15(\mathrm{t}, J=10.8 \mathrm{~Hz}, 1 \mathrm{H})$; ${ }^{13} \mathrm{C} \mathrm{NMR}\left(\mathrm{CDCl}_{3}, 150 \mathrm{MHz}\right) \delta 139.57,129.34,128.90,128.67,81.38,79.95,74.48,71.85,71.31,71.19,70.95$.

2,3,6-Tri-O-benzyl-1,5-anhydro-D-glucitol (13) [49]; Compound 2 (860 mg, $2.0 \mathrm{mmol}$ ) in $10 \mathrm{~mL}$ of DCM was added triethylsilane $(1.6 \mathrm{~mL}, 10 \mathrm{mmol})$ and tifluoroacetic acid $(0.8 \mathrm{~mL}, 10 \mathrm{mmol})$ at $0{ }^{\circ} \mathrm{C}$. The reaction solution was stirred at $\mathrm{rt}$ for $15 \mathrm{~h}$. After the addition $5 \mathrm{~mL}$ of saturated aq. $\mathrm{NaHCO}_{3}$, the mixture was extracted with DCM $(3 \times 30 \mathrm{~mL})$. The organic layer was washed with brine, dried over $\mathrm{Na}_{2} \mathrm{SO}_{4}$, filtered and concentrated under reduced pressure. The crude product was purified by C.C. $(\mathrm{Hex} / \mathrm{EtOAc}=4 / 1)$ to obtain $13(505 \mathrm{mg}, 59 \%)$ as a colorless oil. $[\alpha]_{\mathrm{D}}^{20}=-7.0\left(\mathrm{c} 1.00, \mathrm{CHCl}_{3}\right)$; ${ }^{1} \mathrm{H} \mathrm{NMR}\left(\mathrm{CDCl}_{3}, 600 \mathrm{MHz}\right) \delta 7.37-7.25(\mathrm{~m}, 15 \mathrm{H}), 5.00,4.76(\mathrm{ABq}, J=11.5 \mathrm{~Hz}, 2 \mathrm{H}), 4.69,4.63(\mathrm{ABq}$, $J=11.7 \mathrm{~Hz}, 2 \mathrm{H}), 4.58,4.54(\mathrm{ABq}, J=12.2 \mathrm{~Hz}, 2 \mathrm{H}), 4.04(\mathrm{dd}, J=11.3,5.2 \mathrm{~Hz}, 1 \mathrm{H}), 3.70(\mathrm{dd}, J=10.5$, $3.3 \mathrm{~Hz}, 1 \mathrm{H}), 3.64-3.60(\mathrm{~m}, 2 \mathrm{H}), 3.54(\mathrm{td}, J=9.2,2.1 \mathrm{~Hz}, 1 \mathrm{H}), 3.44(\mathrm{t}, J=8.9 \mathrm{~Hz}, 1 \mathrm{H}), 3.37-3.34(\mathrm{~m}, 1 \mathrm{H})$, $3.23(\mathrm{t}, J=11.0 \mathrm{~Hz}, 1 \mathrm{H}) ;{ }^{13} \mathrm{C} \mathrm{NMR}\left(\mathrm{CDCl}_{3}, 150 \mathrm{MHz}\right) \delta 138.64,138.05,137.84,128.58,128.48,128.39$, $127.95,127.90,127.87,127.83,127.77,127.70,85.40,78.75,78.04,75.10,73.65,73.05,70.87,69.98,68.06$.

2,6-Di-O-benzyl-1,5-anhydro-D-glucitol (14); Compound 3 (860 mg, $2.5 \mathrm{mmol})$ in $15 \mathrm{~mL}$ of DCM was added triethylsilane $(2.0 \mathrm{~mL}, 12.5 \mathrm{mmol})$ and tifluoroacetic acid $(1.0 \mathrm{~mL}, 12.5 \mathrm{mmol})$ at $0{ }^{\circ} \mathrm{C}$. The reaction solution was stirred at $\mathrm{rt}$ for $8 \mathrm{~h}$. After the addition $10 \mathrm{~mL}$ of saturated aq. $\mathrm{NaHCO}_{3}$, the mixture was extracted with DCM $(3 \times 40 \mathrm{~mL})$. The organic layer was washed with brine, dried over $\mathrm{Na}_{2} \mathrm{SO}_{4}$, filtered and concentrated under reduced pressure. The crude product was purified by C.C. $\left(\mathrm{CHCl}_{3} / \mathrm{MeOH}=8 / 1\right)$ to obtain $14(500 \mathrm{mg}, 58 \%)$ as a white solid. $[\alpha]_{\mathrm{D}}^{20}=+17.4$ (c $\left.1.00, \mathrm{CHCl}_{3}\right)$; ${ }^{1} \mathrm{H} \mathrm{NMR}\left(\mathrm{CDCl}_{3}, 600 \mathrm{MHz}\right) \delta 7.36-7.27(\mathrm{~m}, 10 \mathrm{H}), 4.64(\mathrm{~s}, 2 \mathrm{H}), 4.59,4.54(\mathrm{ABq}, J=12.0 \mathrm{~Hz}, 2 \mathrm{H}), 4.02$ $(\mathrm{dd}, J=11.2,5.0 \mathrm{~Hz}, 1 \mathrm{H}), 3.69(\mathrm{dd}, J=10.5,3.6 \mathrm{~Hz}, 1 \mathrm{H}), 3.65(\mathrm{dd}, J=10.5,5.0 \mathrm{~Hz}, 1 \mathrm{H}), 3.55(\mathrm{td}, J=8.8$, $2.1 \mathrm{~Hz}, 1 \mathrm{H}), 3.50(\mathrm{td}, J=9.0,2.6 \mathrm{~Hz}, 1 \mathrm{H}), 3.47-3.43(\mathrm{~m}, 1 \mathrm{H}), 3.37-3.34(\mathrm{~m}, 1 \mathrm{H}), 3.19(\mathrm{t}, J=10.8 \mathrm{~Hz}, 1 \mathrm{H})$; ${ }^{13} \mathrm{C} \mathrm{NMR}\left(\mathrm{CDCl}_{3}, 150 \mathrm{MHz}\right) \delta 137.96,137.71,128.59,128.46,128.07,127.90,127.81,78.31,77.74,77.41$, 
73.68, 72.95, 71.34, 70.01, 67.70. HRMS (ESI, $m / z):[\mathrm{M}+\mathrm{Na}]^{+}$, calcd for $\left[\mathrm{C}_{20} \mathrm{H}_{24} \mathrm{O}_{5} \mathrm{Na}\right]^{+}: 367.1521$; found 367.1521 .

1,5-Anhydro-3,6-di-O-benzyl-D-glucitol (15); Compound $4(680 \mathrm{mg}, 2.0 \mathrm{mmol})$ in $15 \mathrm{~mL}$ of DCM was added triethylsilane $(1.6 \mathrm{~mL}, 10 \mathrm{mmol})$ and trifluoroacetic acid $(0.80 \mathrm{~mL}, 10 \mathrm{mmol})$ at $0{ }^{\circ} \mathrm{C}$. The reaction solution was stirred at $\mathrm{rt}$ for $4 \mathrm{~h}$. After the addition $10 \mathrm{~mL}$ of saturated aq. $\mathrm{NaHCO}_{3}$, the mixture was extracted with DCM $(3 \times 40 \mathrm{~mL})$. The combined organic layer was washed with brine, dried over $\mathrm{Na}_{2} \mathrm{SO}_{4}$, filtered and concentrated under reduced pressure. The crude product was purified by C.C. $\left(\mathrm{CHCl}_{3} / \mathrm{MeOH}=8 / 1\right)$ to obtain $15(420 \mathrm{mg}, 62 \%)$ as a white solid. $[\alpha]_{\mathrm{D}}^{20}=+22.7$ (c 1.00 , $\left.\mathrm{CHCl}_{3}\right) ;{ }^{1} \mathrm{H} \mathrm{NMR}\left(\mathrm{CDCl}_{3}, 600 \mathrm{MHz}\right) \delta 7.39-7.27(\mathrm{~m}, 10 \mathrm{H}), 4.91,4.83(\mathrm{ABq}, J=11.7 \mathrm{~Hz}, 2 \mathrm{H}), 4.59,4.54$ $(\mathrm{ABq}, J=12.2 \mathrm{~Hz}, 2 \mathrm{H}), 3.96(\mathrm{dd}, J=11.2,5.3 \mathrm{~Hz}, 1 \mathrm{H}), 3.72-3.66(\mathrm{~m}, 3 \mathrm{H}), 3.61(\mathrm{dt}, J=9.1,2.6 \mathrm{~Hz}, 1 \mathrm{H})$, 3.39-3.36 (m, 1H), $3.31(\mathrm{t}, J=8.8 \mathrm{~Hz}, 1 \mathrm{H}), 3.21(\mathrm{t}, J=10.8 \mathrm{~Hz}, 1 \mathrm{H}) ;{ }^{13} \mathrm{C} \mathrm{NMR}\left(\mathrm{CDCl}_{3}, 150 \mathrm{MHz}\right) \delta 138.60$, 137.61, 128.70, 128.47, 128.02, 127.93, 127.86, 127.82, 86.47, 78.47, 74.87, 73.74, 72.11, 70.38, 69.79, 69.57. HRMS (ESI, $m / z):[\mathrm{M}+\mathrm{Na}]^{+}$, calcd for $\left[\mathrm{C}_{20} \mathrm{H}_{24} \mathrm{O}_{5} \mathrm{Na}\right]^{+}: 367.1521$; found 367.1522.

1,5-Anhydro-2-O-(3', 4', 5'-tribenzyloxybenzoyl)-3-O-benzyl-4,6-O-benzylidene-D-glucitol (17); Compound 3 (340 mg, $1.0 \mathrm{mmol}$ ), compound 16 [39] (650 mg, $1.5 \mathrm{mmol}), 2$-chloro-1-methylpyridinium iodide ( $380 \mathrm{mg}, 1.5 \mathrm{mmol})$, DMAP ( $37 \mathrm{mg}, 0.30 \mathrm{mmol})$, TEA ( $416 \mu \mathrm{L}, 3.0 \mathrm{mmol})$ in $15 \mathrm{~mL}$ of DCM was stirred at rt for $20 \mathrm{~h}$. After the addition $100 \mathrm{~mL}$ of saturated aq. $\mathrm{NH}_{4} \mathrm{Cl}$, the reaction solution was extracted with $\mathrm{DCM}(3 \times 60 \mathrm{~mL})$. The combined organic layer was washed with brine, dried over $\mathrm{Na}_{2} \mathrm{SO}_{4}$, filtered and concentrated under reduced pressure. The crude was purified by C.C. (DCM/MeOH $=500 / 1-200 / 1)$ to obtain $17(350 \mathrm{mg}, 41 \%)$ as a colorless amorphous oil. $[\alpha]_{\mathrm{D}}^{20}=+8.4\left(\mathrm{c} 1.04, \mathrm{CHCl}_{3}\right) ;{ }^{1} \mathrm{H} \mathrm{NMR}\left(\mathrm{CDCl}_{3}\right.$, $600 \mathrm{MHz}) \delta 7.53-7.11(\mathrm{~m}, 29 \mathrm{H}), 5.61(\mathrm{~s}, 1 \mathrm{H}), 5.27-5.20(\mathrm{~m}, 1 \mathrm{H}), 5.15(\mathrm{~s}, 2 \mathrm{H}), 5.09(\mathrm{~s}, 4 \mathrm{H}), 4.86,4.71(\mathrm{ABq}$, $J=12.0 \mathrm{~Hz}, 2 \mathrm{H}), 4.36(\mathrm{dd}, J=10.3,4.8 \mathrm{~Hz}, 1 \mathrm{H}), 4.19(\mathrm{dd}, J=11.0,5.8 \mathrm{~Hz}, 1 \mathrm{H}), 3.87(\mathrm{t}, J=9.1 \mathrm{~Hz}, 1 \mathrm{H})$, $3.79-3.74(\mathrm{~m}, 2 \mathrm{H}), 3.45(\mathrm{td}, J=9.7,4.9 \mathrm{~Hz}, 1 \mathrm{H}), 3.36(\mathrm{t}, J=10.8 \mathrm{~Hz}, 1 \mathrm{H}) ;{ }^{13} \mathrm{C} \mathrm{NMR}\left(\mathrm{CDCl}_{3}, 150 \mathrm{MHz}\right) \delta$ 165.06, 152.55, 142.74, 138.10, 137.31, 137.28, 136.56, 129.03, 128.57, 128.30, 128.25, 128.22, 128.07, 128.02, $127.89,127.58,127.47,126.02,124.50,109.41,101.32,81.96,79.12,75.14,74.33,71.50,71.30,68.76,67.68$; HRMS (ESI, $m / z):[\mathrm{M}+\mathrm{Na}]^{+}$, calcd for $\left[\mathrm{C}_{48} \mathrm{H}_{44} \mathrm{O}_{9} \mathrm{Na}\right]^{+}:$787.2883; found 787.2881.

1,5-Anhydro-2-O-benzyl-3-O-(3', 4',5'-tribenzyloxybenzoyl)-4,6-O-benzylidene-D-glucitol (18); Compound 2 (0.51 g, $1.5 \mathrm{mmol})$, compound 16 (1.0 g, $2.3 \mathrm{mmol})$, 2-chloro-1-methylpyridinium iodide (0.59 g, $2.3 \mathrm{mmol})$, DMAP ( $28 \mathrm{mg}, 0.23 \mathrm{mmol})$, TEA $(0.62 \mathrm{~mL}, 4.5 \mathrm{mmol})$ in $20 \mathrm{~mL}$ of DCM was stirred at $\mathrm{rt}$ for $20 \mathrm{~h}$. By the same procedure previously described for the preparation of compound 17 , compound $18(0.87 \mathrm{~g}, 68 \%)$ was obtained as a colorless amorphous oil. $[\alpha]_{\mathrm{D}}^{20}=-45.6\left(\mathrm{c} 0.95, \mathrm{CHCl}_{3}\right) ;{ }^{1} \mathrm{H}$ NMR $\left(\mathrm{CDCl}_{3}, 600 \mathrm{MHz}\right)$ 8 7.24-7.44 (m, 24H), 7.20-7.14 (m, 5H), $5.53(\mathrm{t}, J=9.3 \mathrm{~Hz}, 1 \mathrm{H}), 5.46(\mathrm{~s}, 1 \mathrm{H}), 5.15-5.10$ $(\mathrm{m}, 6 \mathrm{H}), 4.55,4.46(\mathrm{ABq}, J=12.4 \mathrm{~Hz}, 2 \mathrm{H}), 4.35(\mathrm{dd}, J=10.5,5.0 \mathrm{~Hz}, 1 \mathrm{H}), 4.13(\mathrm{dd}, J=11.3,5.5 \mathrm{~Hz}, 1 \mathrm{H})$, $3.76-3.72(\mathrm{~m}, 1 \mathrm{H}), 3.70(\mathrm{t}, J=10.3 \mathrm{~Hz}, 1 \mathrm{H}), 3.65(\mathrm{t}, J=9.5 \mathrm{~Hz}, 1 \mathrm{H}), 3.51(\mathrm{td}, J=9.7,4.8 \mathrm{~Hz}, 1 \mathrm{H}), 3.48$ $(\mathrm{t}, J=11.0 \mathrm{~Hz}, 1 \mathrm{H}) ;{ }^{13} \mathrm{C} \mathrm{NMR}\left(\mathrm{CDCl}_{3}, 150 \mathrm{MHz}\right) \delta 165.16,152.47,142.58,137.53,137.39,136.69,128.95$, 128.54, 128.38, 128.21, 128.16, 128.02, 127.98, 127.87, 127.53, 126.14, 125.05, 109.55, 101.35, 79.18, 75.62, 75.15, 74.94, 72.97, 71.48, 71.33, 68.80, 68.74; HRMS (ESI, $m / z):[\mathrm{M}+\mathrm{Na}]^{+}$, calcd for $\left[\mathrm{C}_{48} \mathrm{H}_{44} \mathrm{O} 9 \mathrm{Na}\right]^{+}$: 787.2883; found 787.2882.

1,5-Anhydro-2,3,6-tris-O-benzyl-4-O-(3', 4',5'-tribenzyloxybenzoyl)-D-glucitol (19); Compound 13 ( $340 \mathrm{mg}, 0.78 \mathrm{mmol}$ ), compound 16 (530 mg, $1.2 \mathrm{mmol}$ ), 2-chloro-1-methylpyridinium iodide ( $307 \mathrm{mg}$, $1.2 \mathrm{mmol})$, DMAP ( $95 \mathrm{~g}, 0.78 \mathrm{mmol})$, TEA $(315 \mu \mathrm{L}, 2.3 \mathrm{mmol})$ in $15 \mathrm{~mL}$ of DCM was stirred at rt for $2 \mathrm{~d}$. By the same procedure previously described for the preparation of compound 17 , compound 19 $(607 \mathrm{mg}, 91 \%)$ was obtained as a colorless amorphous oil. $[\alpha]_{\mathrm{D}}^{20}=-32.0\left(\mathrm{c} 0.95, \mathrm{CHCl}_{3}\right) ;{ }^{1} \mathrm{H} \mathrm{NMR}$ $\left(\mathrm{CDCl}_{3}, 600 \mathrm{MHz}\right) \delta$ 7.44-7.26 (m, 20H), 7.23-7.15 (m, 7H), 7.11-7.03 (m, 5H), 5.18-5.15 (m, 3H), $5.13-5.07(\mathrm{~m}, 4 \mathrm{H}), 4.76,4.54(\mathrm{ABq}, J=11.3 \mathrm{~Hz}, 2 \mathrm{H}), 4.74,4.65(\mathrm{ABq}, J=11.7 \mathrm{~Hz}, 1 \mathrm{H}), 4.45(\mathrm{~s}, 2 \mathrm{H}), 4.08$ $(\mathrm{dd}, J=11.3,5.2 \mathrm{~Hz}, 1 \mathrm{H}), 3.75-3.71(\mathrm{~m}, 1 \mathrm{H}), 3.65(\mathrm{t}, J=9.1 \mathrm{~Hz}, 1 \mathrm{H}), 3.59-3.56(\mathrm{~m}, 1 \mathrm{H}), 3.50(\mathrm{dd}, J=10.8$, $2.6 \mathrm{~Hz}, 1 \mathrm{H}), 3.45(\mathrm{dd}, J=10.7,5.8 \mathrm{~Hz}, 1 \mathrm{H}), 3.29(\mathrm{t}, J=11.0 \mathrm{~Hz}, 1 \mathrm{H}) ;{ }^{13} \mathrm{C} \mathrm{NMR}\left(\mathrm{CDCl}_{3}, 150 \mathrm{MHz}\right) \delta$ $164.83,152.41,142.58,138.03,137.58,137.37,136.62,128.57,128.50,128.23,128.14,128.06,128.01,127.94$, $127.86,127.83,127.58,127.49,127.45,124.67,109.34,83.08,78.18,78.08,75.13,74.94,73.67,73.40,71.24$, 69.47, 68.23; HRMS (ESI, $m / z)$ : $[\mathrm{M}+\mathrm{Na}]^{+}$, calcd for $\left[\mathrm{C}_{55} \mathrm{H}_{52} \mathrm{O} \mathrm{O}_{9} \mathrm{Na}\right]^{+}:$879.3509; found 879.3511. 
1,5-Anhydro-2,3,4-tris-O-benzyl-6-O-(3',4',5'-tribenzyloxybenzoyl)-D-glucitol (20); Compound 11 $(0.43 \mathrm{~g}, 1.0 \mathrm{mmol})$, compound $16(0.66 \mathrm{~g}, 1.5 \mathrm{mmol}), 2$-chloro-1-methylpyridinium iodide $(0.38 \mathrm{~g}$, $1.5 \mathrm{mmol})$, DMAP $(0.18 \mathrm{~g}, 1.5 \mathrm{mmol})$, TEA $(0.42 \mathrm{~mL}, 3.0 \mathrm{mmol})$ in $20 \mathrm{~mL}$ of DCM was stirred at $\mathrm{rt}$ for $2 \mathrm{~d}$. By the same procedure previously described for the preparation of compound 17, compound $20(0.50 \mathrm{~g}, 58 \%)$ was obtained as a colorless amorphous oil. $[\alpha]_{\mathrm{D}}^{20}=+27.9\left(\mathrm{c} 1.00, \mathrm{CHCl}_{3}\right) ;{ }^{1} \mathrm{H} \mathrm{NMR}$ $\left(\mathrm{CDCl}_{3}, 600 \mathrm{MHz}\right) \delta 7.43-7.18(\mathrm{~m}, 32 \mathrm{H}), 5.14-5.10(\mathrm{~m}, 6 \mathrm{H}), 5.03,4.88(\mathrm{ABq}, J=10.7 \mathrm{~Hz}, 2 \mathrm{H}), 4,85,4.53$ $(\mathrm{ABq}, J=11.8 \mathrm{~Hz}, 2 \mathrm{H}), 4.74,4.67(\mathrm{ABq}, J=11.7 \mathrm{~Hz}, 2 \mathrm{H}), 4.52(\mathrm{dd}, J=12.0,2.1 \mathrm{~Hz}, 1 \mathrm{H}), 4.37(\mathrm{dd}, J=12.0$, $4.5 \mathrm{~Hz}, 1 \mathrm{H}), 4.03(\mathrm{dd}, J=11.3,5.2 \mathrm{~Hz}, 1 \mathrm{H}), 3.69(\mathrm{t}, J=8.8 \mathrm{~Hz}, 1 \mathrm{H}), 3.66-3.62(\mathrm{~m}, 1 \mathrm{H}), 3.52-3.49(\mathrm{~m}, 1 \mathrm{H})$, $3.46(\mathrm{t}, J=8.9 \mathrm{~Hz}, 1 \mathrm{H}), 3.23(\mathrm{t}, J=10.8 \mathrm{~Hz}, 1 \mathrm{H}) ;{ }^{13} \mathrm{C} \mathrm{NMR}\left(\mathrm{CDCl}_{3}, 150 \mathrm{MHz}\right) \delta 165.82,152.41,142.46$, $138.55,138.04,137.69,137.37,136.65,128.59,128.53,128.48,128.46,128.20,128.08,128.01,127.96,127.93$, $127.85,127.76,127.45,124.91,109.30,86.33,78.48,77.83,77.57,75.73,75.23,75.09,73.29,71.17,68.12$, 63.86; HRMS (ESI, $\mathrm{m} / \mathrm{z}):[\mathrm{M}+\mathrm{Na}]^{+}$, calcd for $\left[\mathrm{C}_{55} \mathrm{H}_{52} \mathrm{O}{ }_{9} \mathrm{Na}\right]^{+}:$: 879.3509; found 879.3509.

1,5-Anhydro-2,3-bis-O-(3', 4', 5'-tribenzyloxybenzoyl)-4,6-O-benzylidene-D-glucitol (21); Compound 1 (380 mg, $1.5 \mathrm{mmol})$, compound $16(2.0 \mathrm{~g}, 4.5 \mathrm{mmol}), 2$-chloro-1-methylpyridinium iodide (1.2 $\mathrm{g}$, $4.5 \mathrm{mmol})$, DMAP $(0.55 \mathrm{~g}, 4.5 \mathrm{mmol})$, TEA $(1.3 \mathrm{~mL}, 9.0 \mathrm{mmol})$ in $20 \mathrm{~mL}$ of DCM was stirred at $\mathrm{rt}$ for $2 \mathrm{~d}$. By the same procedure previously described for the preparation of compound 17, compound 21 $(1.6 \mathrm{~g}, 88 \%)$ was obtained as a colorless amorphous oil. $[\alpha]_{\mathrm{D}}^{20}=+46.4\left(\mathrm{c} 1.00, \mathrm{CHCl}_{3}\right) ;{ }^{1} \mathrm{H} \mathrm{NMR}\left(\mathrm{CDCl}_{3}\right.$, $600 \mathrm{MHz}) \delta 7.44-7.42(\mathrm{~m}, 6 \mathrm{H}), 7.34-7.31(\mathrm{~m}, 26 \mathrm{H}), 7.25-7.22(\mathrm{~m}, 4 \mathrm{H}), 5.80(\mathrm{t}, J=9.5 \mathrm{~Hz}, 1 \mathrm{H}), 5.56(\mathrm{~s}, 1 \mathrm{H})$, $5.27-5.23(\mathrm{~m}, 1 \mathrm{H}), 5.11-4.93(\mathrm{~m}, 12 \mathrm{H}), 4.45-4.41(\mathrm{~m}, 2 \mathrm{H}), 3.87(\mathrm{t}, J=16.0 \mathrm{~Hz}, 1 \mathrm{H}), 3.82(\mathrm{t}, J=17.0 \mathrm{~Hz}$, $1 \mathrm{H}), 3.65-3.62(\mathrm{~m}, 1 \mathrm{H}), 3.55(\mathrm{t}, J=10.5 \mathrm{~Hz}, 1 \mathrm{H}) ;{ }^{13} \mathrm{C} \mathrm{NMR}\left(\mathrm{CDCl}_{3}, 150 \mathrm{MHz}\right) \delta 165.6,165.4,152.6,142.9$, $142.8,137.4,136.8,136.5,129.1,128.5,128.4,128.3,128.2,128.1,128.0,127.5,126.2,109.3,109.1,101.6$, 78.8, 75.1, 73.0, 71.9, 71.2, 71.2, 71.1, 68.7, 67.7; HRMS (ESI, $m / z):[\mathrm{M}+\mathrm{Na}]^{+}$, calcd for $\left[\mathrm{C}_{48} \mathrm{H}_{44} \mathrm{O} 9 \mathrm{Na}\right]^{+}$: 1119.3932; found 1119.3901 .

1,5-Anhydro-2,4-bis-O-(3', 4' , 5'-tribenzyloxybenzoyl)-3,6-bis-O-benzyl-D-glucitol (22); Compound 15 (0.30 mg, $0.87 \mathrm{mmol})$, compound $16(1.3 \mathrm{~g}, 3.0 \mathrm{mmol})$, 2-chloro-1-methylpyridinium iodide ( $0.77 \mathrm{~g}$, $3.0 \mathrm{mmol})$, DMAP $(0.37 \mathrm{~g}, 3.0 \mathrm{mmol})$, TEA $(0.83 \mathrm{~mL}, 6.0 \mathrm{mmol})$ in $20 \mathrm{~mL}$ of DCM was stirred at $\mathrm{rt}$ for $2 \mathrm{~d}$. By the same procedure previously described for the preparation of compound 17 , compound $22(0.93 \mathrm{~g}, 77 \%)$ was obtained as a colorless amorphous oil. $[\alpha]_{\mathrm{D}}^{20}=+7.87\left(\mathrm{c} 1.00, \mathrm{CHCl}_{3}\right) ;{ }^{1} \mathrm{H}$ NMR ${ }^{1} \mathrm{H}$ NMR $\left(\mathrm{CDCl}_{3}, 600 \mathrm{MHz}\right) \delta$ 7.58-7.14 (m, 45H), 7.08-6.95 (m, 5H), 5.32-5.25 (m, 2H), 5.19-5.08 $(\mathrm{m}, 13 \mathrm{H}), 4.61-4.45(\mathrm{~m}, 4 \mathrm{H}), 4.29(\mathrm{dd}, J=11.3,5.5 \mathrm{~Hz}, 1 \mathrm{H}), 3.94(\mathrm{t}, J=9.1 \mathrm{~Hz}, 1 \mathrm{H}), 3.72-3.68(\mathrm{~m}, 1 \mathrm{H})$, 3.58-3.53 (m, 2H), $3.39(\mathrm{t}, J=10.8 \mathrm{~Hz}, 1 \mathrm{H}) ;{ }^{13} \mathrm{C} \mathrm{NMR}\left(\mathrm{CDCl}_{3}, 150 \mathrm{MHz}\right) \delta 164.87,164.71,152.61,152.51$, $142.88,142.79,137.54,137.52,137.32$, 137.27, 136.56, 136.49, 128.59, 128.57, 128.55, 128.28, 128.24, 128.22, 128.16, 128.10, 128.05, 128.02, 127.85, 127.76, 127.65, 127.53, 127.49, 124.51, 109.43, 80.72, 78.29, 75.16, 74.16, 73.72, 72.12, 71.35, 71.32, 69.44, 67.03; HRMS (ESI, $m / z):[\mathrm{M}+\mathrm{Na}]^{+}$, calcd for $\left[\mathrm{C}_{76} \mathrm{H}_{68} \mathrm{O}_{13} \mathrm{Na}\right]^{+}$: 1211.4558; found 1211.4554.

1,5-Anhydro-2,6-bis-O-(3', 4', 5'-tribenzyloxybenzoyl)-3,4-bis-O-benzyl-D-glucitol (23); Compound 10 ( $0.45 \mathrm{~g}, 1.3 \mathrm{mmol})$, compound 16 ( $1.8 \mathrm{~g}, 4.0 \mathrm{mmol})$, 2-chloro-1-methylpyridinium iodide $(1.0 \mathrm{~g}, 4.0 \mathrm{mmol})$, DMAP $(0.49 \mathrm{~g}, 4.0 \mathrm{mmol})$, TEA $(1.1 \mathrm{~mL}, 8.0 \mathrm{mmol})$ in $30 \mathrm{~mL}$ of DCM was stirred at $\mathrm{rt}$ for $2 \mathrm{~d}$. By the same procedure previously described for the preparation of compound 17, compound $23(1.4 \mathrm{~g}, 92 \%)$ was obtained as a colorless amorphous oil. $[\alpha]_{\mathrm{D}}^{20}=+51.2\left(\mathrm{c} 1.00, \mathrm{CHCl}_{3}\right) ;{ }^{1} \mathrm{H} \mathrm{NMR}\left(\mathrm{CDCl}_{3}, 600 \mathrm{MHz}\right)$ 8 7.43-7.18 (m, 44H), 5.27-5.21 (m, 1H), 5.17-5.07 (m, 12H), 4.82, 4.52 (ABq, $J=11.0 \mathrm{~Hz}, 2 \mathrm{H}), 4.77$, $4.70(\mathrm{ABq}, J=11.3 \mathrm{~Hz}, 2 \mathrm{H}), 4.56(\mathrm{~m}, 1 \mathrm{H}), 4.42-4.39(\mathrm{~m}, 1 \mathrm{H}), 4.19(\mathrm{dd}, J=11.2,5.7 \mathrm{~Hz}, 1 \mathrm{H}), 3.85-3.82$ $(\mathrm{m}, 1 \mathrm{H}), 3.60-3.56(\mathrm{~m}, 2 \mathrm{H}), 3.31(\mathrm{t}, J=10.7 \mathrm{~Hz}, 1 \mathrm{H}) ;{ }^{13} \mathrm{C} \mathrm{NMR}\left(\mathrm{CDCl}_{3}, 150 \mathrm{MHz}\right) \delta 165.80,165.05,152.56$, 152.43, 142.76, 142.47, 137.88, 137.49, 137.37, 137.28, 136.66, 136.53, 128.62, 128.60, 128.57, 128.51, 128.39, $128.21,128.12,128.06,128.01,127.88,127.80,127.40,127.37,124.80,124.49,109.32,109.25,84.32,77.91$, 77.42, 75.45, 75.26, 75.13, 75.09, 72.22, 71.26, 71.15, 67.11, 63.52; HRMS (ESI, $m / z):[\mathrm{M}+\mathrm{Na}]^{+}$, calcd for $\left[\mathrm{C}_{76} \mathrm{H}_{68} \mathrm{O}_{13} \mathrm{Na}\right]^{+}:$1211.4558; found 1211.4550.

1,5-Anhydro-2,6-bis-O-benzyl-3,4-bis-O-(3', 4',5'-tribenzyloxybenzoyl)-D-glucitol (24); Compound 14 (380 mg, $1.1 \mathrm{mmol})$, compound 16 (1.5 g, $3.3 \mathrm{mmol})$, 2-chloro-1-methylpyridinium iodide (0.84 g, $3.3 \mathrm{mmol})$, DMAP $(0.91 \mathrm{~g}, 3.3 \mathrm{mmol})$, TEA $(0.91 \mathrm{~mL}, 6.6 \mathrm{mmol})$ in $30 \mathrm{~mL}$ of DCM was stirred at $\mathrm{rt}$ for 
$2 \mathrm{~d}$. By the same procedure previously described for the preparation of compound 17, compound 24 $(1.2 \mathrm{~g}, 92 \%)$ was obtained as a colorless amorphous oil. $[\alpha]_{\mathrm{D}}^{20}=-80.8$ (c $\left.1.00, \mathrm{CHCl}_{3}\right) ;{ }^{1} \mathrm{H} \mathrm{NMR}\left(\mathrm{CDCl}_{3}\right.$, $600 \mathrm{MHz}) \delta 7.41-7.14(\mathrm{~m}, 44 \mathrm{H}), 5.58(\mathrm{t}, J=9.5 \mathrm{~Hz}, 1 \mathrm{H}), 5.31(\mathrm{t}, J=9.8 \mathrm{~Hz}, 1 \mathrm{H}), 5.09-5.00(\mathrm{~m}, 12 \mathrm{H})$, $4.54,4.48(\mathrm{ABq}, J=12.2 \mathrm{~Hz}, 2 \mathrm{H}), 4.52,4.47(\mathrm{ABq}, J=12.0 \mathrm{~Hz}, 2 \mathrm{H}), 4.19(\mathrm{dd}, J=11.5,5.3 \mathrm{~Hz}, 1 \mathrm{H}), 3.79$ $(\mathrm{td}, J=9.9,5.2 \mathrm{~Hz}, 1 \mathrm{H}), 3.73(\mathrm{~m}, 1 \mathrm{H}), 3.59(\mathrm{dd}, J=10.7,2.4 \mathrm{~Hz}, 1 \mathrm{H}), 3.51(\mathrm{dd}, J=10.8,5.3 \mathrm{~Hz}, 1 \mathrm{H}), 3.46$ $(\mathrm{t}, J=11.0 \mathrm{~Hz}, 1 \mathrm{H}) ;{ }^{13} \mathrm{C} \mathrm{NMR}\left(\mathrm{CDCl}_{3}, 150 \mathrm{MHz}\right) \delta 165.62,165.18,152.43,142.67,142.53,137.56,137.48$, 137.43, 137.39, 136.57, 136.56, 128.49, 128.41, 128.38, 128.36, 128.27, 128.16, 127.98, 127.89, 127.87, 127.84, 127.65, 127.55, 124.67, 124.20, 109.15, 77.99, 76.35, 75.22, 75.10, 75.08, 73.70, 72.96, 71.12, 70.07, 69.06, 68.30; HRMS (ESI, $m / z):[\mathrm{M}+\mathrm{Na}]^{+}$, calcd for $\left[\mathrm{C}_{76} \mathrm{H}_{68} \mathrm{O}_{13} \mathrm{Na}\right]^{+}:$1211.4558; found 1211.4558.

1,5-Anhydro-2,4-bis-O-benzyl-3,6-bis-O-(3', 4', 5'-tribenzyloxybenzoyl)-D-glucitol (25); Compound 9 (0.52 mg, $1.5 \mathrm{mmol})$, compound 16 (2.0 g, $4.5 \mathrm{mmol})$, 2-chloro-1-methylpyridinium iodide (1.2 g, $4.5 \mathrm{mmol})$, DMAP $(0.55 \mathrm{~g}, 4.5 \mathrm{mmol})$, TEA $(1.2 \mathrm{~mL}, 9.0 \mathrm{mmol})$ in $20 \mathrm{~mL}$ of DCM was stirred at rt for $2 \mathrm{~d}$. By the same procedure previously described for the preparation of compound 17, compound $25(1.6 \mathrm{~g}, 91 \%)$ was obtained as a colorless amorphous oil. $[\alpha]_{\mathrm{D}}^{20}=+16.1$ (c $\left.1.00, \mathrm{CHCl}_{3}\right) ;{ }^{1} \mathrm{H} \mathrm{NMR}$ $\left(\mathrm{CDCl}_{3}, 600 \mathrm{MHz}\right) \delta 7.43-7.24(\mathrm{~m}, 34 \mathrm{H}), 7.18-7.05(\mathrm{~m}, 10 \mathrm{H}), 5.50(\mathrm{t}, J=9.3 \mathrm{~Hz}, 1 \mathrm{H}), 5.19-5.08(\mathrm{~m}, 12 \mathrm{H})$, $4.56,4.43(\mathrm{ABq}, J=12.4 \mathrm{~Hz}, 2 \mathrm{H}), 4.55(\mathrm{dd}, J=12.0 \mathrm{~Hz}, 2.0 \mathrm{~Hz}, 1 \mathrm{H}), 4.45(\mathrm{dd}, J=12.0,5.5 \mathrm{~Hz}, 1 \mathrm{H}) 4.41$ $(\mathrm{s}, 2 \mathrm{H}), 4.11(\mathrm{dd}, J=11.3,5.2 \mathrm{~Hz}, 1 \mathrm{H}), 3.67-3.62(\mathrm{~m}, 2 \mathrm{H}), 3.54(\mathrm{t}, J=9.5 \mathrm{~Hz}, 1 \mathrm{H}), 3.36(\mathrm{t}, J=11.0 \mathrm{~Hz}, 1 \mathrm{H})$; ${ }^{13} \mathrm{C} \mathrm{NMR}\left(\mathrm{CDCl}_{3}, 150 \mathrm{MHz}\right) \delta 165.84,165.22,152.49,142.66,137.65,137.31,136.99,136.59,128.56,128.36$, 128.22, 128.16, 128.05, 127.94, 127.83, 127.52, 127.47, 124.96, 124.88, 109.54, 109.51, 78.19, 77.87, 76.42, 75.26, 75.13, 74.68, 72.63, 71.32, 67.97, 63.91; HRMS (ESI, $m / z):[\mathrm{M}+\mathrm{Na}]^{+}$, calcd for $\left[\mathrm{C}_{76} \mathrm{H}_{68} \mathrm{O}_{13} \mathrm{Na}^{+}\right.$: 1211.4558; found 1211.4557.

1,5-Anhydro-2,3-bis-O-benzyl-4,6-bis-O-(3', $4^{\prime}, 5^{\prime}$-tribenzyloxybenzoyl)-D-glucitol (26); Compound 5 (0.34 g, $1.0 \mathrm{mmol})$, compound 16 (1.3 g, $3.0 \mathrm{mmol}), 2$-chloro-1-methylpyridinium iodide (0.77 g, $3.0 \mathrm{mmol})$, DMAP $(0.37 \mathrm{~g}, 3.0 \mathrm{mmol})$, TEA $(0.83 \mathrm{~mL}, 6.0 \mathrm{mmol})$ in $20 \mathrm{~mL}$ of DCM was stirred at rt for $2 \mathrm{~d}$. By the same procedure previously described for the preparation of compound 17, compound 26 $(1.0 \mathrm{~g}, 87 \%)$ was obtained as a colorless amorphous oil. $[\alpha]_{\mathrm{D}}^{20}=+13.7$ (c 1.00, $\left.\mathrm{CHCl}_{3}\right) ;{ }^{1} \mathrm{H} \mathrm{NMR}\left(\mathrm{CDCl}_{3}\right.$, $600 \mathrm{MHz}) \delta 7.45-7.21(\mathrm{~m}, 39 \mathrm{H}), 7.12-7.03(\mathrm{~m}, 5 \mathrm{H}), 5.32(\mathrm{t}, J=9.5 \mathrm{~Hz}, 1 \mathrm{H}), 5.15-5.08(\mathrm{~m}, 8 \mathrm{H}), 5.03-5.01$ $(\mathrm{m}, 4 \mathrm{H}), 4.79,4.58(\mathrm{ABq}, J=11.5,2 \mathrm{H}), 4.76,4.66(\mathrm{ABq}, J=11.5 \mathrm{~Hz}, 2 \mathrm{H}), 4.63(\mathrm{dd}, J=12.0,2.8 \mathrm{~Hz}, 1 \mathrm{H})$, $4.12(\mathrm{dd}, J=12.2,5.3 \mathrm{~Hz}, 1 \mathrm{H}), 4.07(\mathrm{dd}, J=11.5,5.0 \mathrm{~Hz}, 1 \mathrm{H}), 3.70-3.68(\mathrm{~m}, 2 \mathrm{H}), 3.70(\mathrm{t}, J=8.9 \mathrm{~Hz}, 1 \mathrm{H})$, $3.30(\mathrm{t}, J=10.8 \mathrm{~Hz}, 1 \mathrm{H}) ;{ }^{13} \mathrm{C} \mathrm{NMR}\left(\mathrm{CDCl}_{3}, 150 \mathrm{MHz}\right) \delta 165.80,164.76,152.48,152.44,142.77,142.42$, 137.98, 137.91, 137.47, 137.37, 136.73, 136.56, 128.53, 128.50, 128.47, 128.20, 128.17, 128.07, 128.03, 128.01, $127.94,127.84,127.60,127.53,124.75,124.54,109.40,109.14,82.95,78.22,76.56,75.15,75.08,73.47,71.24$, 71.06, 70.96, 68.32, 63.44; HRMS (ESI, $m / z):[\mathrm{M}+\mathrm{Na}]^{+}$, calcd for $\left[\mathrm{C}_{76} \mathrm{H}_{68} \mathrm{O}_{13} \mathrm{Na}\right]^{+}:$1211.4558; found 1211.4553.

1,5-Anhydro-2,3,4-tris-O-(3',4', 5'-tribenzyloxybenzoyl)-6-O-benzyl-D-glucitol (27); Compound 12 (180 mg, $0.70 \mathrm{mmol})$, compound 16 (1.4 g, $3.2 \mathrm{mmol}), 2$-chloro-1-methylpyridinium iodide (0.82 g, $3.2 \mathrm{mmol})$, DMAP $(0.39 \mathrm{~g}, 3.2 \mathrm{mmol})$, TEA $(0.89 \mathrm{~mL}, 6.4 \mathrm{mmol})$ in $20 \mathrm{~mL}$ of DCM was stirred at rt for $2 \mathrm{~d}$. By the same procedure previously described for the preparation of compound 17, compound $27(0.94 \mathrm{~g}, 91 \%)$ was obtained as a colorless amorphous oil. $[\alpha]_{\mathrm{D}}^{20}=-4.9$ (c $\left.0.65, \mathrm{CHCl}_{3}\right) ;{ }^{1} \mathrm{H} \mathrm{NMR}$ $\left(\mathrm{CDCl}_{3}, 600 \mathrm{MHz}\right) \delta 7.43-7.16(\mathrm{~m}, 56 \mathrm{H}), 5.82(\mathrm{t}, J=9.6 \mathrm{~Hz}, 1 \mathrm{H}), 5.55(\mathrm{t}, J=10.0 \mathrm{~Hz}, 1 \mathrm{H}), 5.29(\mathrm{td}, J=10.0$, $5.5 \mathrm{~Hz}, 1 \mathrm{H}), 5.13-4.96(\mathrm{~m}, 14 \mathrm{H}), 4.90(\mathrm{~s}, 4 \mathrm{H}), 4.58,4.53(\mathrm{ABq}, J=12.0,2 \mathrm{H}),(\mathrm{dd}, J=10.2,5.6 \mathrm{~Hz}, 1 \mathrm{H})$, 3.88-3.84 (m, 1H), $3.67(\mathrm{dd}, J=11.0,2.4 \mathrm{~Hz}, 1 \mathrm{H}), 3.61(\mathrm{dd}, J=10.8,5.3 \mathrm{~Hz}, 1 \mathrm{H}), 3.57(\mathrm{t}, J=10.8 \mathrm{~Hz}$, $1 \mathrm{H}){ }^{13} \mathrm{C} \mathrm{NMR}\left(\mathrm{CDCl}_{3}, 150 \mathrm{MHz}\right) \delta 165.92,165.17,165.02,152.54,152.50,142.88,142.82,142.71,137.44$, $137.34,136.49,136.45,136.36,128.55,128.51,128.39,128.32,128.27,128.17,128.15,128.10,128.06,128.02$, $127.95,127.92,127.89,127.81,127.72,127.56,127.52,124.08,124.03,109.20,109.11,109.02,78.36,75.12$, 75.09, 75.07, 74.65, 73.77, 71.18, 71.10, 71.02, 70.75, 69.71, 69.00, 67.26; HRMS (ESI, m/z): $[\mathrm{M}+\mathrm{Na}]^{+}$, calcd for $\left[\mathrm{C}_{97} \mathrm{H}_{84} \mathrm{O}_{17} \mathrm{Na}\right]^{+}:$1543.5606; found 1543.5601 .

1,5-Anhydro-2,3,6-tris-O-(3',4', 5'-tribenzyloxybenzoyl)-4-O-benzyl-D-glucitol (28); Compound 8 (0.38 mg, $1.5 \mathrm{mmol})$, compound 16 (3.0 g, mmol), 2-chloro-1-methylpyridinium iodide (1.8 g, $7.0 \mathrm{mmol}), \mathrm{DMAP}$ $(0.12 \mathrm{~g}, 1.0 \mathrm{mmol}), \mathrm{TEA}(1.9 \mathrm{~mL}, 14 \mathrm{mmol})$ in $30 \mathrm{~mL}$ of DCM was stirred at $\mathrm{rt}$ for $1 \mathrm{~d}$. By the same procedure 
previously described for the preparation of compound 17, compound 28 ( $1.0 \mathrm{~g}, 45 \%$ yield) was obtained as a colorless amorphous oil. $[\alpha]_{\mathrm{D}}^{20}=+47.2\left(\mathrm{c} 0.40, \mathrm{CHCl}_{3}\right) ;{ }^{1} \mathrm{H} \mathrm{NMR}\left(\mathrm{CDCl}_{3}, 600 \mathrm{MHz}\right) \delta 7.47-7.19$ $(\mathrm{m}, 51 \mathrm{H}), 7.11-7.05(\mathrm{~m}, 5 \mathrm{H}), 5.75(\mathrm{t}, J=9.1 \mathrm{~Hz}, 1 \mathrm{H}), 5.19-5.03(\mathrm{~m}, 15 \mathrm{H}), 5.00,4.92(\mathrm{ABq}, J=11.7 \mathrm{~Hz}$, $4 \mathrm{H}), 4.60-4.53(\mathrm{~m}, 2 \mathrm{H}), 4.45(\mathrm{~s}, 2 \mathrm{H}), 4.41(\mathrm{dd}, J=11.3,5.5 \mathrm{~Hz}, 1 \mathrm{H}), 3.75-3.70(\mathrm{~m}, 2 \mathrm{H}), 3.46(\mathrm{t}, J=10.8 \mathrm{~Hz}$, $1 \mathrm{H}) ;{ }^{13} \mathrm{C} \mathrm{NMR}\left(\mathrm{CDCl}_{3}, 150 \mathrm{MHz}\right) \delta 165.84,165.51,165.41,152.59,152.54,142.94,142.75,142.65,137.35$, $137.32,137.27,136.86,136.62,136.51,136.39,128.60,128.54,128.46,128.44,128.38,128.22,128.18,128.14$, 128.08, 128.06, 128.01, 127.97, 127.90, 127.61, 127.50, 127.43, 124.81, 124.40, 124.05, 109.54, 109.29, 109.03, 78.11, 76.54, 76.04, 75.14, 75.11, 75.09, 74.96, 71.34, 71.20, 71.06, 70.81, 67.07, 63.58; HRMS (ESI, m/z): $[\mathrm{M}+\mathrm{Na}]^{+}$, calcd for $\left[\mathrm{C}_{97} \mathrm{H}_{84} \mathrm{O}_{17} \mathrm{Na}\right]^{+}:$1543.5606; found 1543.5604 .

1,5-Anhydro-2,4,6-tris-O-(3', 4', ,5'-tribenzyloxybenzoyl)-3-O-benzyl-D-glucitol (29); Compound 7 (0.33 mg, $1.3 \mathrm{mmol})$, compound 16 (2.6 g, $6.0 \mathrm{mmol})$, 2-chloro-1-methylpyridinium iodide (1.5 g, 6.0 mmol), DMAP $(0.73 \mathrm{~g}, 6.0 \mathrm{mmol})$, TEA $(1.7 \mathrm{~mL}, 12 \mathrm{mmol})$ in $20 \mathrm{~mL}$ of DCM was stirred at rt for $2 \mathrm{~d}$. By the same procedure previously described for the preparation of compound 17, compound 29 (1.3 g, 65\% yield) was obtained as a colorless amorphous oil. $[\alpha]_{\mathrm{D}}^{20}=+26.7\left(\mathrm{c} 0.95, \mathrm{CHCl}_{3}\right) ;{ }^{1} \mathrm{H} \mathrm{NMR}\left(\mathrm{CDCl}_{3}, 600 \mathrm{MHz}\right) \delta$ 7.46-7.21 (m, 49H), 7.07-6.96 (m, 5H), $5.48(\mathrm{t}, J=9.5 \mathrm{~Hz}, 1 \mathrm{H}), 5.34-5.29(\mathrm{~m}, 1 \mathrm{H}), 5.17-5.07(\mathrm{~m}, 14 \mathrm{H}), 5.04$ $(\mathrm{s}, 4 \mathrm{H}), 4.68(\mathrm{dd}, J=12.0,3.1 \mathrm{~Hz}, 1 \mathrm{H}), 4.62,4.54(\mathrm{ABq}, J=11.7 \mathrm{~Hz}, 2 \mathrm{H}), 4.30(\mathrm{dd}, J=11.2,5.7 \mathrm{~Hz}, 1 \mathrm{H})$, $4.22(\mathrm{dd}, J=12.2,5.0 \mathrm{~Hz}, 1 \mathrm{H}), 3.99(\mathrm{t}, J=9.1 \mathrm{~Hz}, 1 \mathrm{H}), 3.90-3.87(\mathrm{~m}, 1 \mathrm{H}), 3.42(\mathrm{t}, J=10.8 \mathrm{~Hz}, 1 \mathrm{H}) ;{ }^{13} \mathrm{C}$ $\mathrm{NMR}\left(\mathrm{CDCl}_{3}, 150 \mathrm{MHz}\right) \delta 165.81,164.83,164.61,152.63,152.55,152.46,142.94,142.49,137.46,137.41$, 137.33, 137.24, 136.67, 136.50, 136.46, 128.57, 128.53, 128.51, 128.46, 128.21, 128.19, 128.14, 128.10, 128.06, $128.02,127.95,127.85,127.80,127.62,127.56,127.47,124.64,124.41,124.38,109.47,109.44,109.13,80.62$, 76.53, 75.16, 75.08, 74.34, 72.02, 71.35, 71.30, 71.05, 70.98, 67.05, 63.33; HRMS (ESI, m/z): $[\mathrm{M}+\mathrm{Na}]^{+}$, calcd for $\left[\mathrm{C}_{97} \mathrm{H}_{84} \mathrm{O}_{17} \mathrm{Na}\right]^{+}:$1543.5606; found 1543.5599 .

1,5-Anhydro-2-O-benzyl-3,4,6-tris-O-(3',4',5'-tribenzyloxybenzoyl)-D-glucitol (30); Compound 6 (0.38 g, $1.5 \mathrm{mmol})$, compound 16 (3.0 g, $6.8 \mathrm{mmol})$, 2-chloro-1-methylpyridinium iodide $(1.7 \mathrm{~g}, 6.8 \mathrm{mmol})$, DMAP $(0.83 \mathrm{~g}, 6.8 \mathrm{mmol})$, TEA $(2.0 \mathrm{~mL}, 14 \mathrm{mmol})$ in $30 \mathrm{~mL}$ of DCM was stirred at $\mathrm{rt}$ for 2 days. By the same procedure previously described for the preparation of compound 17, compound 30 ( $2.0 \mathrm{~g}, 89 \%$ yield) was obtained as a colorless amorphous oil. $[\alpha]_{\mathrm{D}}^{20}=-36.2$ (c $\left.1.00, \mathrm{CHCl}_{3}\right) ;{ }^{1} \mathrm{H} \mathrm{NMR}\left(\mathrm{CDCl}_{3}, 600 \mathrm{MHz}\right) \delta$ 7.42-7.14 (m, 54H), $5.67(\mathrm{t}, J=9.6 \mathrm{~Hz}, 1 \mathrm{H}), 5.41(\mathrm{t}, J=9.8 \mathrm{~Hz}, 1 \mathrm{H}), 5.13-4.94(\mathrm{~m}, 18 \mathrm{H}), 4.69(\mathrm{dd}, J=12.2$, $2.9 \mathrm{~Hz}, 1 \mathrm{H}), 4.59,4.51(\mathrm{ABq}, J=12.4 \mathrm{~Hz}, 2 \mathrm{H}), 4.23(\mathrm{dd}, J=12.4,5.5 \mathrm{~Hz}, 1 \mathrm{H}), 4.19(\mathrm{dd}, J=11.5,5.3 \mathrm{~Hz}$, 1H) $3.92(\mathrm{~m}, 1 \mathrm{H}), 3.83(\mathrm{td}, J=9.8,5.3 \mathrm{~Hz}, 1 \mathrm{H}), 3.48(\mathrm{t}, J=11.0 \mathrm{~Hz}, 1 \mathrm{H}) ;{ }^{13} \mathrm{C} \mathrm{NMR}\left(\mathrm{CDCl}_{3}, 150 \mathrm{MHz}\right) \delta$ $165.68,165.65,165.29,152.51,152.48,152.40,142.87,142.66,142.53,137.54,137.43,137.38,136.63,136.46$, $128.51,128.46,128.43,128.38,128.29,128.15,128.11,127.98,127.92,127.89,127.87,127.80,127.78,127.57$, 127.52, 124.65, 124.53, 123.97, 109.18, 76.69, 76.16, 75.17, 75.08, 72.95, 71.13, 71.03, 70.20, 68.38, 63.54; HRMS (ESI, $m / z):[\mathrm{M}+\mathrm{Na}]^{+}$, calcd for $\left[\mathrm{C}_{97} \mathrm{H}_{84} \mathrm{O}_{17} \mathrm{Na}\right]^{+}:$1543.5606; found 1543.5607.

1,5-Anhydro-2-O-(3', $4^{\prime}, 5^{\prime}$-trihydroxybenzoyl)-D-glucitol (31) [31]; $\mathrm{Pd}(\mathrm{OH})_{2}$ on $\mathrm{C}(20 \mathrm{wt} . \%, 20 \mathrm{mg})$ was added to a solution of $17(270 \mathrm{mg}, 0.35 \mathrm{mmol})$ in $10 \mathrm{~mL}$ of $\mathrm{MeOH}$ and $10 \mathrm{~mL}$ of THF under the argon. After the replaced argon atmosphere to hydrogen gas, the suspension was stirred at rt for $6 \mathrm{~h}$. The reaction mixture was filtered and concentrated under reduced pressure to obtain purple amorphous oil. The purple amorphous oil was dissolved by $2 \mathrm{~mL}$ acetone and filtered through a whatman ${ }^{\mathrm{TM}}$ puradisc $0.1 \mu \mathrm{M}$ TF and concentrated under reduced pressure. In addition, the purple amorphous oil dissolved with $2 \mathrm{~mL}$ of $\mathrm{MeOH}$ and added acidic resin until becoming a clear solution. After the filtered through the whatman ${ }^{\mathrm{TM}}$ puradisc $0.1 \mu \mathrm{m} \mathrm{TF}$, the solution was concentrated under reduced pressure to give $31(106 \mathrm{mg}, 96 \%)$ as pale yellow amorphous oil. $[\alpha]_{\mathrm{D}}^{20}=+58.5$ (c $\left.0.70, \mathrm{MeOH}\right)$; ${ }^{1} \mathrm{H}$ NMR (CD $\left.\mathrm{OD}, 600 \mathrm{MHz}\right) \delta 7.08(\mathrm{~d}, J=5.5 \mathrm{~Hz}, 2 \mathrm{H}), 4.87-4.83$ (overlap, $\left.1 \mathrm{H}\right), 4.08$ (dd, $J=10.5,5.5 \mathrm{~Hz}$, $1 \mathrm{H}), 3.86(\mathrm{dd}, J=11.9,2.2 \mathrm{~Hz}, 1 \mathrm{H}), 3.66-3.63(\mathrm{~m}, 2 \mathrm{H}), 3.36(\mathrm{t}, J=9.5 \mathrm{~Hz}, 1 \mathrm{H}), 3.29(\mathrm{t}, J=10.7 \mathrm{~Hz}, 1 \mathrm{H})$, $3.24(\mathrm{ddd}, J=9.7,5.9,2.3 \mathrm{~Hz}, 1 \mathrm{H}) .{ }^{13} \mathrm{C} \mathrm{NMR}\left(\mathrm{CD}_{3} \mathrm{OD}, 150 \mathrm{MHz}\right) \delta 167.80,146.42,139.92,121.14,110.25$, 82.56, 77.06, 73.28, 71.97, 67.84, 62.93; HRMS (ES $\left.{ }^{-}, \mathrm{m} / \mathrm{z}\right)$ : $[\mathrm{M}-\mathrm{H}]^{-}$, calcd for $\left[\mathrm{C}_{13} \mathrm{H}_{15} \mathrm{O}_{9}\right]^{-}: 315.0716$; found 315.0718. Please find ${ }^{1} \mathrm{H}$ NMR, ${ }^{13} \mathrm{C}$ NMR spectra of compounds 31-44, 54-58, 61 and 67 in the Supplementary Materials. 
1,5-Anhydro-3-O-(3', 4' , 5'-trihydroxybenzoyl)-D-glucitol (32) [29]; $\mathrm{Pd}(\mathrm{OH})_{2}$ on $\mathrm{C}$ (20 wt.\%, $\left.20 \mathrm{mg}\right)$ was added to a solution of compound $18(270 \mathrm{mg}, 0.35 \mathrm{mmol})$ in $10 \mathrm{~mL}$ of $\mathrm{MeOH}$ and $10 \mathrm{~mL}$ of THF under the argon. By the same procedure previously described for the preparation of compound 31, desired compound $32\left(110 \mathrm{mg}\right.$, quant.) was obtained as a colorless amorphous oil. $[\alpha]_{\mathrm{D}}^{20}=+24.8$ (c $1.0 \mathrm{MeOH}) ;{ }^{1} \mathrm{H}$ NMR CD $\left.\mathrm{CD}_{3} \mathrm{OD}, 600 \mathrm{MHz}\right) \delta 7.13(\mathrm{~s}, 2 \mathrm{H}), 5.04(\mathrm{t}, J=9.3 \mathrm{~Hz}, 1 \mathrm{H}), 3.97(\mathrm{dd}, J=11.3,5.5 \mathrm{~Hz}$, $1 \mathrm{H}), 3.85(\mathrm{dd}, J=11.9,2.2 \mathrm{~Hz}, 1 \mathrm{H}), 3.73-3.69(\mathrm{~m}, 1 \mathrm{H}), 3.66(\mathrm{dd}, J=12.0,5.8 \mathrm{~Hz}, 1 \mathrm{H}), 3.51(\mathrm{t}, J=9.5 \mathrm{~Hz}$, 1H), 3.31-3.27 (overlap, 2H). ${ }^{13} \mathrm{C}$ NMR $\left(\mathrm{CD}_{3} \mathrm{OD}, 150 \mathrm{MHz}\right) \delta 168.50,146.39,139.64,121.88,110.34,82.51$, 81.20, 70.95, 69.98, 69.89, 62.75; HRMS (ES $\left.{ }^{-}, m / z\right):[\mathrm{M}-\mathrm{H}]^{-}$, calcd for $\left[\mathrm{C}_{13} \mathrm{H}_{15} \mathrm{O}_{9}\right]^{-}:$315.0716; found 315.0721.

1,5-Anhydro-4-O-(3', $4^{\prime}, 5^{\prime}$-trihydroxybenzoyl)-D-glucitol (33) [29]; $\mathrm{Pd}(\mathrm{OH})_{2}$ on C (20 wt.\%, $\left.20 \mathrm{mg}\right)$ was added to a solution of compound $19(280 \mathrm{mg}, 0.33 \mathrm{mmol})$ in $10 \mathrm{~mL}$ of $\mathrm{MeOH}$ and $10 \mathrm{~mL}$ of THF under the argon. By the same procedure previously described for the preparation of compound 31, desired compound $33(100 \mathrm{mg}, 96 \%)$ was obtained as a colorless amorphous oil. $[\alpha]_{\mathrm{D}}^{20}=-5.7$ (c 0.90 , $\mathrm{MeOH}) ;{ }^{1} \mathrm{H}$ NMR (Acetone $\left.-d_{6}, 600 \mathrm{MHz}\right) \delta 7.14(\mathrm{~s}, 2 \mathrm{H}), 4.88(\mathrm{t}, J=9.3 \mathrm{~Hz}, 1 \mathrm{H}), 3.94(\mathrm{dd}, J=11.2$, $5.3 \mathrm{~Hz}, 1 \mathrm{H}), 3.68(\mathrm{t}, J=8.9 \mathrm{~Hz}, 1 \mathrm{H}), 3.64-3.60(\mathrm{~m}, 1 \mathrm{H}), 3.57(\mathrm{dd}, J=12.0,2.1 \mathrm{~Hz}, 1 \mathrm{H}), 3.51-3.44(\mathrm{~m}, 2 \mathrm{H})$, $3.25(\mathrm{t}, J=10.8 \mathrm{~Hz}, 1 \mathrm{H}) ;{ }^{13} \mathrm{C}$ NMR (Acetone $\left.-d_{6}, 150 \mathrm{MHz}\right) \delta 166.59,145.90,138.86,121.46,110.07$, 80.56, 77.44, 72.70, 71.38, 70.45, 62.54; HRMS $\left(\mathrm{ES}^{-}, \mathrm{m} / \mathrm{z}\right)$ : $[\mathrm{M}-\mathrm{H}]^{-}$, calcd for $\left[\mathrm{C}_{13} \mathrm{H}_{15} \mathrm{O}_{9}\right]^{-}:$: 315.0716; found 315.0718 .

1,5-Anhydro-6-O-(3' , 4' ,5'-trihydroxybenzoyl)-D-glucitol (34) [31]; $\mathrm{Pd}(\mathrm{OH})_{2}$ on C (20 wt.\%, $\left.20 \mathrm{mg}\right)$ was added to a solution of compound $20(210 \mathrm{mg}, 0.25 \mathrm{mmol})$ in $10 \mathrm{~mL}$ of $\mathrm{MeOH}$ and $10 \mathrm{~mL}$ of THF under the argon. By the same procedure previously described for the preparation of compound 31, desired compound $55(74 \mathrm{mg}, 96 \%)$ was obtained as a colorless amorphous oil. $[\alpha]_{\mathrm{D}}^{20}=+30.3$ (c 1.00, $\mathrm{MeOH}) ;{ }^{1} \mathrm{H}$ NMR $\left(\mathrm{CD}_{3} \mathrm{OD}, 600 \mathrm{MHz}\right) \delta 7.09(\mathrm{~s}, 2 \mathrm{H}), 4.54(\mathrm{dd}, J=11.9,1.9 \mathrm{~Hz}, 1 \mathrm{H}), 4.35(\mathrm{dd}, J=12.0$, $5.5 \mathrm{~Hz}, 1 \mathrm{H}), 3.93(\mathrm{dd}, J=11.2,5.3 \mathrm{~Hz}, 1 \mathrm{H}), 3.51-3.55(\mathrm{~m}, 1 \mathrm{H}), 3.48-3.45(\mathrm{~m}, 1 \mathrm{H}), 3.40(\mathrm{t}, J=8.6 \mathrm{~Hz}$, $1 \mathrm{H}), 3.37(\mathrm{t}, J=8.6 \mathrm{~Hz}, 1 \mathrm{H}), 3.23(\mathrm{t}, J=10.8 \mathrm{~Hz}, 1 \mathrm{H}) .{ }^{13} \mathrm{C} \mathrm{NMR}\left(\mathrm{CD}_{3} \mathrm{OD}, 150 \mathrm{MHz}\right) \delta 168.32,146.36$,

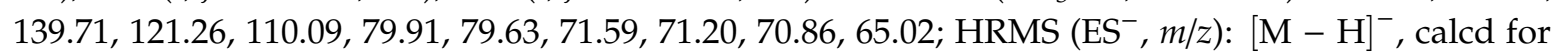
$\left[\mathrm{C}_{13} \mathrm{H}_{15} \mathrm{O}_{9}\right]^{-}:$:315.0716; found 315.0718.

1,5-Anhydro-2,3-bis-O-(3', 4', 5'-trihydroxybenzoyl)-D-glucitol (35) [29]; $\mathrm{Pd}(\mathrm{OH})_{2}$ on C (20 wt.\%, $50 \mathrm{mg}$ ) was added to a solution of compound $21(210 \mathrm{mg}, 0.25 \mathrm{mmol})$ in $10 \mathrm{~mL}$ of $\mathrm{MeOH}$ and $10 \mathrm{~mL}$ of THF under the argon. By the same procedure previously described for the preparation of compound 31, desired compound 35 (313 mg, quant.) was obtained as a colorless amorphous oil. $[\alpha]_{\mathrm{D}}^{20}=+139.7$ (c 1.00, MeOH); ${ }^{1} \mathrm{H}$ NMR $\left(\mathrm{CD}_{3} \mathrm{OD}, 600 \mathrm{MHz}\right) \delta 7.05(\mathrm{~s}, 2 \mathrm{H}), 6.96(\mathrm{~s}, 2 \mathrm{H}), 5.41(\mathrm{t}, J=9.5 \mathrm{~Hz}, 1 \mathrm{H}), 5.08$ $(\mathrm{td}, J=10.1,5.3 \mathrm{~Hz}, 1 \mathrm{H}), 4.20(\mathrm{dd}, J=11.0,5.5 \mathrm{~Hz}, 1 \mathrm{H}), 3.90(\mathrm{dd}, J=11.9,2.2 \mathrm{~Hz}, 1 \mathrm{H}), 3.74-3.69(\mathrm{~m}, 2 \mathrm{H})$, $3.45(\mathrm{t}, J=10.8 \mathrm{~Hz}, 1 \mathrm{H}), 3.40(\mathrm{ddd}, J=9.6,5.5,2.1 \mathrm{~Hz}, 1 \mathrm{H}) ;{ }^{13} \mathrm{C} \mathrm{NMR}\left(\mathrm{CD}_{3} \mathrm{OD}, 150 \mathrm{MHz}\right) \delta 168.18$, 167.38, 146.38, 146.34, 140.08, 139.85, 121.29, 120.55, 110.30, 110.25, 82.65, 77.91, 71.39, 69.83, 67.81, 62.57; HRMS (ES,$m / z):[\mathrm{M}-\mathrm{H}]^{-}$, calcd for $\left[\mathrm{C}_{20} \mathrm{H}_{19} \mathrm{O}_{13}\right]^{-}: 467.0826$; found 467.0831 .

1,5-Anhydro-2,4-bis-O-(3', 4', 5'-trihydroxybenzoyl)-D-glucitol (36) [29]; $\mathrm{Pd}(\mathrm{OH})_{2}$ on C (20 wt.\%, $50 \mathrm{mg}$ ) was added to a solution of compound $22(680 \mathrm{mg}, 0.57 \mathrm{mmol})$ in $20 \mathrm{~mL}$ of $\mathrm{MeOH}$ and $20 \mathrm{~mL}$ of THF under the argon. By the same procedure previously described for the preparation of compound 31, desired compound 36 (267 mg, quant.) was obtained as a colorless amorphous oil. $[\alpha]_{\mathrm{D}}^{20}=+11.3(\mathrm{c} 0.70, \mathrm{MeOH}) ;{ }^{1} \mathrm{H}$ NMR (Acetone $\left.-d_{6}, 600 \mathrm{MHz}\right) \delta 7.17(\mathrm{~s}, 2 \mathrm{H}), 7.14(\mathrm{dd}, J=9.8$, $4.0 \mathrm{~Hz}, 2 \mathrm{H}), 5.04(\mathrm{t}, J=9.5 \mathrm{~Hz}, 1 \mathrm{H}), 5.01-4.97(\mathrm{~m}, 1 \mathrm{H}), 4.15-4.11(\mathrm{~m}, 2 \mathrm{H}), 3.63-3.54(\mathrm{~m}, 3 \mathrm{H}), 3.41$ $(\mathrm{t}, J=10.8 \mathrm{~Hz}, 1 \mathrm{H}) ;{ }^{13} \mathrm{C}$ NMR (Acetone $\left.-d_{6}, 150 \mathrm{MHz}\right) \delta 166.39,166.21,146.00,145.98,138.99,138.94$, 121.43, 121.38, 110.15, 110.09, 80.72, 74.30, 73.19, 72.73, 67.38, 62.49; HRMS (ES $\left.^{-}, \mathrm{m} / z\right)$ : $[\mathrm{M}-\mathrm{H}]^{-}$, calcd for $\left[\mathrm{C}_{20} \mathrm{H}_{19} \mathrm{O}_{13}\right]^{-}: 467.0826$; found 467.0827 .

1,5-Anhydro-2,6-bis-O-(3', 4', 5' -trihydroxybenzoyl)-D-glucitol (37) [26]; $\mathrm{Pd}(\mathrm{OH})_{2}$ on C (20 wt.\%, $\left.50 \mathrm{mg}\right)$ was added to a solution of compound $23(440 \mathrm{mg}, 0.37 \mathrm{mmol})$ in $20 \mathrm{~mL}$ of $\mathrm{MeOH}$ and $20 \mathrm{~mL}$ of THF under the argon. By the same procedure previously described for the preparation of compound 31, desired compound 37 (170 mg, quant.) was obtained as a yellow amorphous oil. $[\alpha]_{\mathrm{D}}^{20}=+19.4$ (c 1.00 in $\mathrm{MeOH}) ;{ }^{1} \mathrm{H}$ NMR (Acetone $\left.-d_{6}, 600 \mathrm{MHz}\right) \delta 7.16(\mathrm{~s}, 2 \mathrm{H}), 7.14(\mathrm{~s}, 2 \mathrm{H}), 4.92-4.88(\mathrm{~m}, 1 \mathrm{H})$, 
$4.57(\mathrm{~d}, J=10.7 \mathrm{~Hz}, 1 \mathrm{H}), 4.41-4.35(\mathrm{~m}, 1 \mathrm{H}), 4.07(\mathrm{dd}, J=10.8,5.3 \mathrm{~Hz}, 1 \mathrm{H}), 3.83-3.78(\mathrm{~m}, 1 \mathrm{H}), 3.61-3.57$ $(\mathrm{m}, 2 \mathrm{H}), 3.37(\mathrm{t}, J=10.7 \mathrm{~Hz}, 1 \mathrm{H}) ;{ }^{13} \mathrm{C}$ NMR (Acetone $\left.-d_{6}, 150 \mathrm{MHz}\right) \delta 166.63,166.30,146.02,145.96$, 138.89, 138.77, 121.74, 121.46, 110.07, 109.85, 79.47, 76.57, 72.94, 71.60, 67.45, 64.54; HRMS (ES ${ }^{-}, \mathrm{m} / \mathrm{z}$ ): $[\mathrm{M}-\mathrm{H}]^{-}$, calcd for $\left[\mathrm{C}_{20} \mathrm{H}_{19} \mathrm{O}_{13}\right]^{-}:$: 467.0826; found 467.0831.

1,5-Anhydro-3,4-bis-O-(3', $4^{\prime}, 5^{\prime}$-trihydroxybenzoyl)-D-glucitol (38); $\mathrm{Pd}(\mathrm{OH})_{2}$ on C (20 wt.\%, $\left.50 \mathrm{mg}\right)$ was added to a solution of compound $24(710 \mathrm{mg}, 0.60 \mathrm{mmol})$ in $20 \mathrm{~mL}$ of $\mathrm{MeOH}$ and $20 \mathrm{~mL}$ of THF under the argon. By the same procedure previously described for the preparation of compound 31, desired compound 38 (280 mg, quant.) was obtained as a yellow amorphous oil. $[\alpha]_{\mathrm{D}}^{20}=-78.2$ (c 1.00, $\mathrm{MeOH}) ;{ }^{1} \mathrm{H}$ NMR (Acetone $\left.-d_{6}, 600 \mathrm{MHz}\right) \delta 7.06(\mathrm{~s}, 2 \mathrm{H}), 7.04(\mathrm{~s}, 2 \mathrm{H}), 5.40(\mathrm{t}, J=9.5 \mathrm{~Hz}, 1 \mathrm{H}), 5.16$ $(\mathrm{t}, J=9.6 \mathrm{~Hz}, 1 \mathrm{H}), 4.06(\mathrm{dd}, J=11.3,5.5 \mathrm{~Hz}, 1 \mathrm{H}), 3.98-3.93(\mathrm{~m}, 1 \mathrm{H}), 3.71-3.64(\mathrm{~m}, 2 \mathrm{H}), 3.58(\mathrm{dd}, J=12.5$, $5.7 \mathrm{~Hz}, 1 \mathrm{H}), 3.45(\mathrm{t}, J=10.8 \mathrm{~Hz}, 1 \mathrm{H}) ;{ }^{13} \mathrm{C}$ NMR (Acetone $\left.-d_{6}, 150 \mathrm{MHz}\right) \delta 166.41,166.12,145.83$, 145.72, 139.04, 138.66, 121.52, 120.78, 110.05, 110.01, 80.29, 78.04, 70.50, 70.17, 69.50, 62.25; HRMS (ES ${ }^{-}$, $m / z):[\mathrm{M}-\mathrm{H}]^{-}$, calcd for $\left[\mathrm{C}_{20} \mathrm{H}_{19} \mathrm{O}_{13}\right]^{-}:$467.0826; found 467.0829.

1,5-Anhydro-3,6-bis-O-(3', 4', 5' -trihydroxybenzoyl)-D-glucitol (39) [35]; $\mathrm{Pd}(\mathrm{OH})_{2}$ on C (20 wt.\%, $50 \mathrm{mg}$ ) was added to a solution of compound $25(800 \mathrm{mg}, 0.67 \mathrm{mmol})$ in $20 \mathrm{~mL}$ of MeOH and $20 \mathrm{~mL}$ of THF under the argon. By the same procedure previously described for the preparation of compound 31, desired compound 39 (310 mg, quant.) was obtained as a yellow amorphous oil. $[\alpha]_{\mathrm{D}}^{20}=+28.7$ (c 0.60 , $\mathrm{MeOH}) ;{ }^{1} \mathrm{H}$ NMR (Acetone $\left.-d_{6}, 600 \mathrm{MHz}\right) \delta 7.17(\mathrm{~s}, 2 \mathrm{H}), 7.16(\mathrm{~s}, 2 \mathrm{H}), 5.11(\mathrm{t}, J=9.1 \mathrm{~Hz}, 1 \mathrm{H}), 4.57$ $(\mathrm{dd}, J=11.9,1.9 \mathrm{~Hz}, 1 \mathrm{H}), 4.40(\mathrm{dd}, J=11.9,5.3 \mathrm{~Hz}, 1 \mathrm{H}), 3.99(\mathrm{dd}, J=11.0,5.5 \mathrm{~Hz}, 1 \mathrm{H}), 3.84-3.79(\mathrm{~m}, 1 \mathrm{H})$, $3.72(\mathrm{t}, J=9.5 \mathrm{~Hz}, 1 \mathrm{H}), 3.65-3.63(\mathrm{~m}, 1 \mathrm{H}), 3.38(\mathrm{t}, J=10.8 \mathrm{~Hz}, 1 \mathrm{H}) ;{ }^{13} \mathrm{C}$ NMR (Acetone $-d_{6}, 150 \mathrm{MHz}$ ) $\delta 166.90,166.63,145.97,145.82,138.71,138.52,122.16,121.76,110.10,109.85,81.10,79.61,70.73,69.66$, 69.46, 64.54; HRMS (ES', $\mathrm{m} / \mathrm{z})$ : $[\mathrm{M}-\mathrm{H}]^{-}$, calcd for $\left[\mathrm{C}_{20} \mathrm{H}_{19} \mathrm{O}_{13}\right]^{-}:$: 467.0826; found 467.0828.

1,5-Anhydro-4,6-bis-O-( $3^{\prime}, 4^{\prime}, 5^{\prime}$-trihydroxybenzoyl)-D-glucitol (40) [35]; $\mathrm{Pd}(\mathrm{OH})_{2}$ on C (20 wt.\%, $\left.50 \mathrm{mg}\right)$ was added to a solution of compound $26(430 \mathrm{mg}, 0.36 \mathrm{mmol})$ in $20 \mathrm{~mL}$ of MeOH and $20 \mathrm{~mL}$ of THF under the argon. By the same procedure previously described for the preparation of compound 31, desired compound $40(160 \mathrm{mg}, 95 \%)$ was obtained as a yellow amorphous oil. $[\alpha]_{\mathrm{D}}^{20}=+41.7$ (c 0.60 , $\mathrm{MeOH}) ;{ }^{1} \mathrm{H}$ NMR (Acetone $\left.-d_{6}, 600 \mathrm{MHz}\right) \delta 7.15(\mathrm{~s}, 2 \mathrm{H}), 7.14(\mathrm{~s}, 2 \mathrm{H}), 5.09(\mathrm{t}, J=9.5 \mathrm{~Hz}, 1 \mathrm{H}), 4.41$ $(\mathrm{dd}, J=12.0,2.1 \mathrm{~Hz}, 1 \mathrm{H}), 4.13(\mathrm{dd}, J=12.0,5.8 \mathrm{~Hz}, 1 \mathrm{H}), 3.97(\mathrm{dd}, J=11.2,5.3 \mathrm{~Hz}, 1 \mathrm{H}), 3.82-3.79(\mathrm{~m}, 1 \mathrm{H})$, $3.75(\mathrm{t}, J=9.1 \mathrm{~Hz}, 1 \mathrm{H}), 3.71-3.66(\mathrm{~m}, 1 \mathrm{H}), 3.33(\mathrm{t}, J=10.8 \mathrm{~Hz}, 1 \mathrm{H}) ;{ }^{13} \mathrm{C}$ NMR (Acetone $-d_{6}, 150 \mathrm{MHz}$ ) $\delta$ 166.47, 166.13, 145.92, 138.90, 138.77, 121.53, 121.38, 110.12, 109.91, 77.56, 77.42, 72.24, 71.28, 70.51, 69.79, 63.98, 55.32; HRMS (ES-, $\mathrm{m} / \mathrm{z})$ : $[\mathrm{M}-\mathrm{H}]^{-}$, calcd for $\left[\mathrm{C}_{20} \mathrm{H}_{19} \mathrm{O}_{13}\right]^{-}:$: 467.0826; found 467.0829.

1,5-Anhydro-2,3,4-tris-O-(3', 4', 5'-trihydroxybenzoyl)-D-glucitol (41) [35]; $\mathrm{Pd}(\mathrm{OH})_{2}$ on C (20 wt.\%, $50 \mathrm{mg}$ ) was added to a solution of compound $27(520 \mathrm{mg}, 0.34 \mathrm{mmol})$ in $20 \mathrm{~mL}$ of $\mathrm{MeOH}$ and $20 \mathrm{~mL}$ of THF under the argon. By the same procedure previously described for the preparation of compound 31, desired compound 41 (210 mg, quant.) was obtained as a yellow amorphous oil. $[\alpha]_{\mathrm{D}}^{20}=-4.8$ (c 1.00, $\mathrm{MeOH}) ;{ }^{1} \mathrm{H}$ NMR (Acetone $\left.-d_{6}, 600 \mathrm{MHz}\right) \delta 7.07(\mathrm{~d}, J=3.8 \mathrm{~Hz}, 4 \mathrm{H}), 7.01(\mathrm{~d}, J=3.8 \mathrm{~Hz}, 2 \mathrm{H}), 5.77$ $(\mathrm{t}, J=9.6 \mathrm{~Hz}, 1 \mathrm{H}), 5.36(\mathrm{t}, J=9.8 \mathrm{~Hz}, 1 \mathrm{H}), 5.23(\mathrm{td}, J=10.1,5.4 \mathrm{~Hz}, 1 \mathrm{H}), 4.28(\mathrm{dd}, J=11.2,5.7 \mathrm{~Hz}, 1 \mathrm{H})$, $3.83-3.80(\mathrm{~m}, 1 \mathrm{H}), 3.72(\mathrm{dd}, J=12.4,2.1 \mathrm{~Hz}, 1 \mathrm{H}), 3.62-3.65(\mathrm{~m}, 2 \mathrm{H}) ;{ }^{13} \mathrm{C}$ NMR (Acetone $-d_{6}, 150 \mathrm{MHz}$ ) $\delta$ 166.12, 165.91, 165.89, 145.80, 145.65, 139.00, 138.76, 120.93, 120.70, 120.62, 110.03, 109.96, 109.91, 80.37, 74.46, 70.83, 69.96, 67.27, 61.99; HRMS (ES $\left.{ }^{-}, \mathrm{m} / z\right)$ : $[\mathrm{M}-\mathrm{H}]^{-}$, calcd for $\left[\mathrm{C}_{27} \mathrm{H}_{23} \mathrm{O}_{17}\right]^{-}:$619.0935; found 619.0934.

1,5-Anhydro-2,3,6-tris-O-(3',4',5'-trihydroxybenzoyl)-D-glucitol (42) [30]; $\mathrm{Pd}(\mathrm{OH})_{2}$ on C (20 wt.\%, $50 \mathrm{mg}$ ) was added to a solution of compound $28(410 \mathrm{mg}, 0.27 \mathrm{mmol})$ in $20 \mathrm{~mL}$ of $\mathrm{MeOH}$ and $20 \mathrm{~mL}$ of THF under the argon. By the same procedure previously described for the preparation of compound 31, desired compound 42 (170 $\mathrm{mg}$, quant.) was obtained as a yellow amorphous oil. $[\alpha]_{\mathrm{D}}^{20}=+80.9(\mathrm{c} 0.70, \mathrm{MeOH}) ;{ }^{1} \mathrm{H}$ NMR (Acetone $\left.-d_{6}, 600 \mathrm{MHz}\right) \delta 7.19(\mathrm{~s}, 2 \mathrm{H}), 7.10(\mathrm{~s}, 2 \mathrm{H}), 7.04(\mathrm{~s}, 2 \mathrm{H})$, $5.50(\mathrm{t}, J=9.5 \mathrm{~Hz}, 1 \mathrm{H}), 5.13-5.09(\mathrm{~m}, 1 \mathrm{H}), 4.60(\mathrm{dd}, J=11.9,1.9 \mathrm{~Hz}, 1 \mathrm{H}), 4.47(\mathrm{dd}, J=12.0,4.8 \mathrm{~Hz}$, $1 \mathrm{H}), 4.20(\mathrm{dd}, J=11.0,5.5 \mathrm{~Hz}, 1 \mathrm{H}), 3.93(\mathrm{t}, J=9.5 \mathrm{~Hz}, 1 \mathrm{H}), 3.79-3.77(\mathrm{~m}, 1 \mathrm{H}), 3.56(\mathrm{t}, J=10.8 \mathrm{~Hz}$, $1 \mathrm{H}){ }^{13} \mathrm{C}$ NMR $\left(\right.$ Acetone $\left.-d_{6}, 150 \mathrm{MHz}\right) \delta 166.60,166.39,166.03,146.05,145.96,145.87,139.15,138.86$, 
138.81, 121.64, 121.56, 120.75, 110.06, 110.00, 109.89, 79.67, 77.07, 70.84, 69.60, 67.44, 64.23; HRMS (ES ${ }^{-}$, $\mathrm{m} / \mathrm{z}):[\mathrm{M}-\mathrm{H}]^{-}$, calcd for $\left[\mathrm{C}_{27} \mathrm{H}_{23} \mathrm{O}_{17}\right]^{-}:$: 619.0935; found 619.0931.

1,5-Anhydro-2,4,6-tris-O-(3', 4', 5'-trihydroxybenzoyl)-D-glucitol (43) [29]; $\mathrm{Pd}(\mathrm{OH})_{2}$ on C (20 wt.\%, $50 \mathrm{mg}$ ) was added to a solution of compound $29(480 \mathrm{mg}, 0.32 \mathrm{mmol})$ in $20 \mathrm{~mL}$ of $\mathrm{MeOH}$ and $20 \mathrm{~mL}$ of THF under the argon. B By the same procedure previously described for the preparation of compound 31, desired compound 37 (200 $\mathrm{mg}$, quant.) was obtained as a yellow amorphous oil. $[\alpha]_{\mathrm{D}}^{20}=+36.3(\mathrm{c} 1.00, \mathrm{MeOH}) ;{ }^{1} \mathrm{H}$ NMR (Acetone $\left.-d_{6}, 600 \mathrm{MHz}\right) \delta 7.17(\mathrm{~s}, 2 \mathrm{H}), 7.16(\mathrm{~s}, 2 \mathrm{H}), 7.15(\mathrm{~s}, 2 \mathrm{H})$, $5.25(\mathrm{dd}, J=10.0,9.3 \mathrm{~Hz}, 1 \mathrm{H}), 5.07-5.03(\mathrm{~m}, 1 \mathrm{H}), 4.46(\mathrm{dd}, J=12.2,1.9 \mathrm{~Hz}, 1 \mathrm{H}), 4.20-4.16(\mathrm{~m}, 3 \mathrm{H})$, 3.95-3.92 (m, 1H), $3.50(\mathrm{t}, J=10.8 \mathrm{~Hz}, 1 \mathrm{H}) ;{ }^{13} \mathrm{C}$ NMR (Acetone $\left.-d_{6}, 150 \mathrm{MHz}\right) \delta 166.44,166.19,165.95$, 145.98, 138.98, 138.83, 121.56, 121.35, 121.31, 110.20, 110.11, 109.96, 77.63, 74.27, 72.96, 72.22, 67.45, 63.76; HRMS $\left(\mathrm{ES}^{-}, \mathrm{m} / \mathrm{z}\right):[\mathrm{M}-\mathrm{H}]^{-}$, calcd for $\left[\mathrm{C}_{27} \mathrm{H}_{23} \mathrm{O}_{17}\right]^{-}: 619.0935$; found 619.0934 .

1,5-Anhydro-3,4,6-tris-O-(3', 4', 5'-trihydroxybenzoyl)-D-glucitol (44) [35]; $\mathrm{Pd}(\mathrm{OH})_{2}$ on C (20 wt.\%, $50 \mathrm{mg}$ ) was added to a solution of compound $30(765 \mathrm{mg}, 0.50 \mathrm{mmol})$ in $20 \mathrm{~mL}$ of MeOH and $20 \mathrm{~mL}$ of THF under the argon. By the same procedure previously described for the preparation of compound 31, desired compound 37 (310 mg, quant.) was obtained as a colorless amorphous oil. $[\alpha]_{\mathrm{D}}^{20}=-6.12$ (c 0.80 , $\mathrm{MeOH}) ;{ }^{1} \mathrm{H}$ NMR (Acetone $\left.-d_{6}, 600 \mathrm{MHz}\right) \delta 7.20(\mathrm{~s}, 2 \mathrm{H}), 7.07(\mathrm{~s}, 2 \mathrm{H}), 7.06(\mathrm{~s}, 2 \mathrm{H}), 5.46(\mathrm{t}, J=9.5 \mathrm{~Hz}$, $1 \mathrm{H}), 5.34(\mathrm{t}, J=9.8 \mathrm{~Hz}, 1 \mathrm{H}), 4.47(\mathrm{dd}, J=12.4,2.1 \mathrm{~Hz}, 1 \mathrm{H}), 4.27(\mathrm{dd}, J=12.2,5.3 \mathrm{~Hz}, 1 \mathrm{H}), 4.12(\mathrm{dd}, J=11.3$, $5.5 \mathrm{~Hz}, 1 \mathrm{H}), 4.04-4.00(\mathrm{~m}, 2 \mathrm{H}), 3.55(\mathrm{t}, J=10.8 \mathrm{~Hz}, 1 \mathrm{H}){ }^{13} \mathrm{C}$ NMR (Acetone $\left.-d_{6}, 150 \mathrm{MHz}\right) \delta 166.39$, 166.35, 165.72, 145.83, 145.73, 145.66, 138.98, 138.74, 138.64, 121.44, 121.38, 120.67, 110.05, 109.97, 109.94, 77.86, 77.41, 70.54, 69.73, 69.41, 63.52; HRMS (ES $\left.{ }^{-}, m / z\right):[\mathrm{M}-\mathrm{H}]^{-}$, calcd for $\left[\mathrm{C}_{27} \mathrm{H}_{23} \mathrm{O}_{17}\right]^{-}:$619.0935; found 619.0941 .

3,4-bis(Benzyloxy)benzoic acid (45) [51]; Methyl 3,4-dihydroxybenzoate (2.5 g, $15 \mathrm{mmol}$ ) and $\mathrm{K}_{2} \mathrm{CO}_{3}$ $(8.2 \mathrm{~g}, 60 \mathrm{mmol})$ and $\mathrm{KI}(2.0 \mathrm{~g}, 12 \mathrm{mmol})$ in $100 \mathrm{~mL}$ of acetone was stirred at rt. The reaction suspension was slowly added $\mathrm{BnCl}(4.2 \mathrm{~mL}, 36 \mathrm{mmol})$ and refluxed for $7 \mathrm{~h}$. TLC indicated full conversion of the start material, added $\mathrm{MeOH}$ and stirred $1 \mathrm{~h}$. The reaction mixture was filtered by celite and filtrate was evaporated under reduced pressure. The residue was purified by recrystallization with hexane, and the mother liquid was purified by C.C (Hex/EtOAc $=100 / 1-4 / 1)$ to afford methyl ester of 45 (total $5.0 \mathrm{~g}$, $97 \%)$ as a white solid. Further on, methyl ester of 45 (3.0 g, $9.0 \mathrm{mmol}), \mathrm{KOH}(5.0 \mathrm{~g}, 90 \mathrm{mmol})$ in $90 \mathrm{~mL}$ of 1,4-dioxane and $30 \mathrm{~mL}$ of $\mathrm{MeOH}$ was stirred at $85^{\circ} \mathrm{C}$ for $2 \mathrm{~h}$. TLC indicated full conversion of the start material, the reaction mixture was cooled to $0{ }^{\circ} \mathrm{C}$ and slowly added $6 \mathrm{M} \mathrm{HCl}$ until $\mathrm{pH}=1$. The precipitating muddy suspension was filtrated, and the white residue was washed by water and $\mathrm{MeOH}$ until $\mathrm{pH}=7$. The white solid was dried in vacuo, purified by recrystallizing with $\mathrm{MeOH}$ to obtain desired compound $45(2.4 \mathrm{~g}, 79 \%)$ as colorless needles. m.p. $211^{\circ} \mathrm{C} ;{ }^{1} \mathrm{H}$ NMR (DMSO- $d 6$, $600 \mathrm{MHz}) \delta 7.56-7.15(\mathrm{~m}, 13 \mathrm{H}), 5.22(\mathrm{~s}, 2 \mathrm{H}), 5.18(\mathrm{~s}, 2 \mathrm{H}) ;{ }^{13} \mathrm{C}$ NMR (DMSO - d6, $\left.150 \mathrm{MHz}\right) \delta 166.88$, 151.94, 147.50, 136.93, 136.62, 128.36, 128.30, 127.82, 127.71, 127.46, 127.34, 123.36, 123.22, 114.43, 112.97, $69.87,69.73$.

3,5-bis(Benzyloxy)benzoic acid (46) [53]; Methyl 3,5-dihydroxybenzoate (2.5 g, $15 \mathrm{mmol}$ ) and $\mathrm{K}_{2} \mathrm{CO}_{3}$ $(8.2 \mathrm{~g}, 60 \mathrm{mmol})$ and $\mathrm{KI}(2.0 \mathrm{~g}, 12 \mathrm{mmol})$ in $100 \mathrm{~mL}$ of acetone was stirred at rt. The reaction suspension was slowly added $\mathrm{BnCl}(4.2 \mathrm{~mL}, 36 \mathrm{mmol})$ and refluxed for $9 \mathrm{~h}$. By the same procedure previously described for the preparation of compound 45, methyl ester of $\mathbf{4 6}$ (5.2 g, quant.) was obtained as a white solid. Further on, methyl ester of 46 ( $3.0 \mathrm{~g}$, $9.0 \mathrm{mmol}), \mathrm{KOH}(5.0 \mathrm{~g}, 90 \mathrm{mmol})$ in $90 \mathrm{~mL}$ of 1,4-dioxane and $30 \mathrm{~mL}$ of $\mathrm{MeOH}$ was stirred at $85^{\circ} \mathrm{C}$ for $2 \mathrm{~h}$. By the same procedure previously described for the preparation of compound 45 , desire compound $46(2.5 \mathrm{~g}, 81 \%)$ was obtained as colorless needles. m.p. $185^{\circ} \mathrm{C} ;{ }^{1} \mathrm{H}$ NMR (DMSO - d6, $\left.600 \mathrm{MHz}\right) \delta 7.46-7.30(\mathrm{~m}, 11 \mathrm{H}), 7.19-7.16(\mathrm{~m}, 2 \mathrm{H}), 6.93-6.93(\mathrm{~m}, 1 \mathrm{H})$, $5.15(\mathrm{~s}, 4 \mathrm{H}) ;{ }^{13} \mathrm{C}$ NMR (DMSO - d6, $\left.150 \mathrm{MHz}\right) \delta 166.80,159.30,136.64,132.80,128.36,127.79,127.58$, $107.87,106.43,69.38$.

3-(Benzyloxy)benzoic acid (47) [52]; Methyl 3-hydroxybenzoate (2.3 g, $15 \mathrm{mmol})$ and $\mathrm{K}_{2} \mathrm{CO}_{3}(4.2 \mathrm{~g}$, $30 \mathrm{mmol})$ and $\mathrm{KI}(1.0 \mathrm{~g}, 6.0 \mathrm{mmol})$ in $100 \mathrm{~mL}$ of acetone was stirred at rt. The reaction suspension was slowly added $\mathrm{BnCl}(2.1 \mathrm{~mL}, 18 \mathrm{mmol})$ and refluxed for $10 \mathrm{~h}$. By the same procedure previously described for the preparation of compound 45 , methyl ester of 47 (3.7 g, 95\%) was obtained as a white 
solid. Further on, methyl ester of 47 (1.9 g, $7.4 \mathrm{mmol}), \mathrm{KOH}(4.2 \mathrm{~g}, 74 \mathrm{mmol})$ in $90 \mathrm{~mL}$ of 1,4-dioxane and $30 \mathrm{~mL}$ of $\mathrm{MeOH}$ was stirred at $85^{\circ} \mathrm{C}$ for $2 \mathrm{~h}$. By the same procedure previously described for the preparation of compound procedure described for previously 45 preparation, desire compound 47 $(1.4 \mathrm{~g}, 85 \%)$ was obtained as colorless needles. m.p. $136{ }^{\circ} \mathrm{C} ;{ }^{1} \mathrm{H}$ NMR $(600 \mathrm{MHz}$, DMSO-d6) $87.57-7.53$ (m, 2H), 7.48-7.39 (m, 5H), 7.35-7.32 (m, 1H), 7.28-7.26 (m, 1H), $5.17(\mathrm{~s}, 2 \mathrm{H}) ;{ }^{13} \mathrm{C}-\mathrm{NMR}(150 \mathrm{MHz}$, DMSO-d6) $\delta 167.12,158.34,136.84,132.23,129.79,128.50,127.92,127.70,121.82,119.77,114.89,69.35$.

4-(Benzyloxy)benzoic acid (48) [51]; Methyl 4-hydroxybenzoate (2.3 g, $15 \mathrm{mmol})$ and $\mathrm{K}_{2} \mathrm{CO}_{3}(4.2 \mathrm{~g}$, $30 \mathrm{mmol})$ and $\mathrm{KI}(1.0 \mathrm{~g}, 6.0 \mathrm{mmol})$ in $100 \mathrm{~mL}$ of acetone was stirred at rt. The reaction suspension was slowly added $\mathrm{BnCl}(2.1 \mathrm{~mL}, 18 \mathrm{mmol})$ and refluxed for $9 \mathrm{~h}$. By the same procedure previously described for the preparation of compound 45 , methyl ester of 48 ( $3.6 \mathrm{~g}$, quant.) was obtained as a white solid. Further on, methyl ester of 48 (2.3 g, $9.0 \mathrm{mmol}), \mathrm{KOH}(5.0 \mathrm{~g}, 90 \mathrm{mmol})$ in $90 \mathrm{~mL}$ of 1,4-dioxane and $30 \mathrm{~mL}$ of $\mathrm{MeOH}$ was stirred at $85^{\circ} \mathrm{C}$ for $2 \mathrm{~h}$. By the same procedure previously described for the preparation of compound procedure described for previously 45 preparation, desired compound 48 (1.4 g, 85\%) was obtained as colorless needles. m.p. $192{ }^{\circ} \mathrm{C} ;{ }^{1} \mathrm{H}$ NMR $(600 \mathrm{MHz}$, DMSO-d6) $87.95-7.90$ $(\mathrm{m}, 2 \mathrm{H}), 7.48-7.46(\mathrm{~m}, 2 \mathrm{H}), 7.42-7.40(\mathrm{~m}, 2 \mathrm{H}), 7.36-7.34(\mathrm{~m}, 1 \mathrm{H}), 7.12-7.09(\mathrm{~m}, 2 \mathrm{H}), 5.19(\mathrm{~s}, 2 \mathrm{H}) ;{ }^{13} \mathrm{C}-\mathrm{NMR}$ (150 MHz, DMSO-d6) $\delta 166.90,161.83,136.43,131.27,128.40,127.92,127.73,123.09,114.50,69.35$.

1,5-Anhydro-2,3,4,6-tetrakis-O-(3', 4', 5'-tribenzyloxybenzoyl)-D-glucitol (49) [50]; 1,5-AG (82 mg, $0.50 \mathrm{mmol})$, compound 16 (1.1 g, $2.4 \mathrm{mmol})$, 2-chloro-1-methylpyridinium iodide ( $0.61 \mathrm{~g}, 2.4 \mathrm{mmol})$, $\operatorname{DMAP}(0.29 \mathrm{~g}, 2.4 \mathrm{mmol})$, TEA $(0.67 \mathrm{~mL}, 4.8 \mathrm{mmol})$ in $20 \mathrm{~mL}$ of DCM was stirred at $\mathrm{rt}$ for $2 \mathrm{~d}$. By the same procedure previously described for the preparation of compound 17, desire compound $49(0.86 \mathrm{~g}$, $92 \%)$ was obtained as a colorless amorphous oil. $[\alpha]_{\mathrm{D}}^{20}=+7.0\left(\mathrm{c} 1.00, \mathrm{CHCl}_{3}\right) ;{ }^{1} \mathrm{H} \mathrm{NMR}(600 \mathrm{MHz}$, CHLOROFORM-D) $\delta 7.56-7.07(\mathrm{~m}, 68 \mathrm{H}), 5.89(\mathrm{t}, J=9.8 \mathrm{~Hz}, 1 \mathrm{H}), 5.63(\mathrm{t}, J=9.8 \mathrm{~Hz}, 1 \mathrm{H}), 5.35-5.31$ $(\mathrm{m}, 1 \mathrm{H}), 5.18-4.94(\mathrm{~m}, 22 \mathrm{H}), 4.88-4.84(\mathrm{~m}, 4 \mathrm{H}), 4.76(\mathrm{dd}, J=12.2,2.9 \mathrm{~Hz}, 1 \mathrm{H}), 4.52(\mathrm{dd}, J=11.3,5.5 \mathrm{~Hz}$, $1 \mathrm{H}), 4.30(\mathrm{dd}, J=12.4,5.2 \mathrm{~Hz}, 1 \mathrm{H}), 4.06-4.03(\mathrm{~m}, 1 \mathrm{H}), 3.60(\mathrm{t}, J=10.8 \mathrm{~Hz}, 1 \mathrm{H}) ;{ }^{13} \mathrm{C} \mathrm{NMR}\left(\mathrm{CDCl}_{3}\right.$, $150 \mathrm{MHz}) \delta 165.91,165.71,165.14,165.10,152.54,152.43,143.06,142.93,142.79,142.62,137.41,137.31$, $136.59,136.44,136.35,136.25,128.54,128.45,128.44,128.37,128.27,128.23,128.14,128.11,128.08$, 128.02, 128.00, 127.95, 127.90, 127.87, 127.81, 127.55, 127.52, 124.56, 123.95, 123.79, 109.22, 109.14, 109.03, 75.10, 75.06, 74.53, 71.18, 71.11, 71.05, 70.51, 69.78, 67.31, 63.36 HRMS (ESI, $\mathrm{m} / \mathrm{z}):[\mathrm{M}+\mathrm{Na}]^{+}$, calcd for $\left[\mathrm{C}_{118} \mathrm{H}_{100} \mathrm{O}_{21} \mathrm{Na}\right]^{+}:$: 1875.6644; found 1875.6653.

1,5-Anhydro-2,3,4,6-tetrakis-O-(3', 4'-dibenzyloxybenzoyl)-D-glucitol (50); 1,5-AG (0.12 g, 0.7 mmol), compound 45 (1.4 g, $4.2 \mathrm{mmol})$, 2-chloro-1-methylpyridinium iodide (1.1 g, $4.2 \mathrm{mmol})$, DMAP (0.52 g, $4.2 \mathrm{mmol})$, TEA $(1.1 \mathrm{~mL}, 8.0 \mathrm{mmol})$ in $20 \mathrm{~mL}$ of DCM was stirred at $\mathrm{rt}$ for $2 \mathrm{~d}$. By the same procedure previously described for the preparation of compound 17, desired compound $50(0.61 \mathrm{~g}, 72 \%)$ was obtained as a colorless amorphous oil. $[\alpha]_{\mathrm{D}}^{20}=+18.8\left(\mathrm{c} 0.90, \mathrm{CHCl}_{3}\right) ;{ }^{1} \mathrm{H} \mathrm{NMR}\left(\mathrm{CDCl}_{3}, 600 \mathrm{MHz}\right) \delta$ 7.65-7.23 (m, 48H), 6.89-6.86 (m, 2H), 6.82-6.80 (m, 1H), 6.77-6.75 (m, 1H), $5.81(\mathrm{t}, J=9.8 \mathrm{~Hz}, 1 \mathrm{H}), 5.56$ $(\mathrm{t}, J=9.6 \mathrm{~Hz}, 1 \mathrm{H}), 5.29(\mathrm{td}, J=10.1,5.5 \mathrm{~Hz}, 1 \mathrm{H}), 5.22-5.03(\mathrm{~m}, 14 \mathrm{H}), 4.99(\mathrm{~s}, 2 \mathrm{H}), 4.63(\mathrm{dd}, J=12.4$, $2.7 \mathrm{~Hz}, 1 \mathrm{H}), 4.43(\mathrm{dd}, J=11.3,5.5 \mathrm{~Hz}, 1 \mathrm{H}), 4.29(\mathrm{dd}, J=12.4,5.2 \mathrm{~Hz}, 1 \mathrm{H}), 3.97-3.94(\mathrm{~m}, 1 \mathrm{H}), 3.53$ $(\mathrm{t}, J=10.8 \mathrm{~Hz}, 1 \mathrm{H}) ;{ }^{13} \mathrm{C} \mathrm{NMR}\left(\mathrm{CDCl}_{3}, 150 \mathrm{MHz}\right) \delta 165.83,165.69,165.17,164.91,153.26,153.14,152.96$, 148.27, 148.17, 136.87, 136.68, 136.51, 136.38, 128.57, 128.53, 128.46, 128.40, 127.94, 127.86, 127.46, 127.41, $127.09,127.01,126.98,124.36,124.25,121.79,115.37,115.20,113.01,76.91,73.90,71.07,71.04,70.95,70.70$, 70.64, 70.16, 69.38, 67.29, 63.16; HRMS (ESI, $m / z):[\mathrm{M}+\mathrm{Na}]^{+}$, calcd for $\left[\mathrm{C}_{62} \mathrm{H}_{56} \mathrm{O}_{13} \mathrm{Na}\right]^{+}:$1451.4980; found 1451.4977 .

1,5-Anhydro-2,3,4,6-tetrakis-O-(3',5'-dibenzyloxybenzoyl)-D-glucitol (51); 1,5-AG (0.15 g, 0.9 mmol), compound 46 (1.8 g, $5.4 \mathrm{mmol})$, 2-chloro-1-methylpyridinium iodide (1.3 g, $5.4 \mathrm{mmol})$, DMAP (0.66 g, $5.4 \mathrm{mmol})$, TEA $(1.5 \mathrm{~mL}, 10.8 \mathrm{mmol})$ in $20 \mathrm{~mL}$ of DCM was stirred at $\mathrm{rt}$ for $2 \mathrm{~d}$. By the same procedure previously described for the preparation of compound 17, desired compound $51(0.69 \mathrm{~g}, 63 \%)$ was obtained as a colorless amorphous oil. $[\alpha]_{\mathrm{D}}^{20}=+8.3\left(\mathrm{c} 0.90, \mathrm{CHCl}_{3}\right) ;{ }^{1} \mathrm{H} \mathrm{NMR}\left(\mathrm{CDCl}_{3}, 600 \mathrm{MHz}\right) \delta$ 7.45-7.18 (m, 48H), 6.79-6.76 (m, 2H), 6.72-6.69 (m, 2H), $5.89(\mathrm{t}, J=9.6 \mathrm{~Hz}, 1 \mathrm{H}), 5.62(\mathrm{t}, J=9.8 \mathrm{~Hz}, 1 \mathrm{H})$, $5.37(\mathrm{td}, J=10.1,5.6 \mathrm{~Hz}, 1 \mathrm{H}), 5.06-4.98(\mathrm{~m}, 8 \mathrm{H}), 4.95-4.84(\mathrm{~m}, 8 \mathrm{H}), 4.66(\mathrm{dd}, J=12.2,2.9 \mathrm{~Hz}, 1 \mathrm{H}), 4.49$ $(\mathrm{dd}, J=11.3,5.5 \mathrm{~Hz}, 1 \mathrm{H}), 4.40(\mathrm{dd}, J=12.2,5.3 \mathrm{~Hz}, 1 \mathrm{H}), 4.04-4.01(\mathrm{~m}, 1 \mathrm{H}), 3.58(\mathrm{t}, J=10.8 \mathrm{~Hz}, 1 \mathrm{H})$; 
${ }^{13} \mathrm{C} \mathrm{NMR}\left(\mathrm{CDCl}_{3}, 150 \mathrm{MHz}\right) \delta 165.84,165.80,165.20,165.06,159.76,159.75,159.70,136.44,136.31,136.26$, $136.22,131.45,130.91,130.87,130.71,128.60,128.57,128.51,128.09,128.06,127.59,127.57,108.53,108.49$, 108.41, 108.11, 107.74, 107.70, 107.51, 74.28, 70.41, 70.23, 70.19, 69.73, 67.21, 63.51; HRMS (ESI, m/z): $[\mathrm{M}+\mathrm{Na}]^{+}$, calcd for $\left[\mathrm{C}_{62} \mathrm{H}_{56} \mathrm{O}_{13} \mathrm{Na}\right]^{+}: 1451.4980$; found 1451.4980 .

1,5-Anhydro-2,3,4,6-tetrakis-O-(3'-benzyloxybenzoyl)-D-glucitol (52); 1,5-AG (0.10 g, $0.6 \mathrm{mmol})$, compound $47(0.82 \mathrm{~g}, 3.6 \mathrm{mmol})$, 2-chloro-1-methylpyridinium iodide $(0.81 \mathrm{~g}, 3.6 \mathrm{mmol})$, $\operatorname{DMAP}(0.44 \mathrm{~g}$, $3.6 \mathrm{mmol}), \mathrm{TEA}(1.0 \mathrm{~mL}, 7.2 \mathrm{mmol})$ in $20 \mathrm{~mL}$ of DCM was stirred at $\mathrm{rt}$ for $2 \mathrm{~d}$. By the same procedure previously described for the preparation of compound 17, desire compound $52(0.56 \mathrm{~g}, 92 \%)$ was obtained as a colorless amorphous oil. $[\alpha]_{\mathrm{D}}^{20}=+22.7\left(\mathrm{c} 1.00, \mathrm{CHCl}_{3}\right) ;{ }^{1} \mathrm{H} \mathrm{NMR}\left(\mathrm{CDCl}_{3}, 600 \mathrm{MHz}\right) \delta$ $7.68-7.05(\mathrm{~m}, 36 \mathrm{H}), 5.90(\mathrm{t}, J=9.6 \mathrm{~Hz}, 1 \mathrm{H}), 5.65(\mathrm{t}, J=9.8 \mathrm{~Hz}, 1 \mathrm{H}), 5.40(\mathrm{td}, J=10.1,5.6 \mathrm{~Hz}, 1 \mathrm{H}), 5.02-5.13$ $(\mathrm{m}, 4 \mathrm{H}), 5.00(\mathrm{~s}, 2 \mathrm{H}), 4.96(\mathrm{~s}, 2 \mathrm{H}), 4.65(\mathrm{dd}, J=12.0,2.7 \mathrm{~Hz}, 1 \mathrm{H}), 4.48(\mathrm{dd}, J=11.2,5.7 \mathrm{~Hz}, 1 \mathrm{H}), 4.42$ $(\mathrm{dd}, J=12.2,5.3 \mathrm{~Hz}, 1 \mathrm{H}), 4.02-4.05(\mathrm{~m}, 1 \mathrm{H}), 3.59(\mathrm{t}, J=10.8 \mathrm{~Hz}, 1 \mathrm{H}) ;{ }^{13} \mathrm{C} \mathrm{NMR}\left(\mathrm{CDCl}_{3}, 150 \mathrm{MHz}\right) \delta$ $166.03,165.85,165.34,165.11,158.66,136.57,136.37,130.91,130.30,130.12,129.56,129.49,128.61,128.56$, 128.13, 127.62, 127.58, 122.52, 121.12, 120.85, 120.71, 120.53, 115.16, 115.12, 115.00, 114.84, 76.89, 74.07, 70.27, 70.12, 69.50, 67.21, 63.31; HRMS (ESI, $m / z):[\mathrm{M}+\mathrm{Na}]^{+}$, calcd for $\left[\mathrm{C}_{62} \mathrm{H}_{56} \mathrm{O}_{13} \mathrm{Na}\right]^{+}: 1027.3306$; found 1027.3301 .

1,5-Anhydro-2,3,4,6-tetrakis-O-(4'-benzyloxybenzoyl)-D-glucitol (53); 1,5-AG (0.16 g, $1.0 \mathrm{mmol})$, compound 48 (1.4 g, $6.1 \mathrm{mmol}), 2$-chloro-1-methylpyridinium iodide (1.4 g, $6.0 \mathrm{mmol})$, DMAP $(0.73 \mathrm{~g}, 6.0 \mathrm{mmol})$, TEA $(1.7 \mathrm{~mL}, 12 \mathrm{mmol})$ in $30 \mathrm{~mL}$ of DCM was stirred at $\mathrm{rt}$ for $2 \mathrm{~d}$. By the same procedure previously described for the preparation of compound 17, desire compound $53(0.99 \mathrm{~g}, 98 \%)$ was obtained as a colorless amorphous oil. $[\alpha]_{\mathrm{D}}^{20}=+38.2\left(\mathrm{c} 0.90, \mathrm{CHCl}_{3}\right) ;{ }^{1} \mathrm{H} \mathrm{NMR}\left(\mathrm{CDCl}_{3}, 600 \mathrm{MHz}\right) \delta$ 8.06-7.96 (m, 2H), 7.92-7.86 (m, 6H), 7.44-7.17 (m, 20H), 6.93-6.78 (m, 8H), $5.89(\mathrm{t}, J=9.5 \mathrm{~Hz}, 1 \mathrm{H}), 5.63$ $(\mathrm{t}, J=9.8 \mathrm{~Hz}, 1 \mathrm{H}), 5.40(\mathrm{td}, J=10.0,5.7 \mathrm{~Hz}, 1 \mathrm{H}), 5.02-4.85(\mathrm{~m}, 8 \mathrm{H}), 4.61-4.59(\mathrm{~m}, 1 \mathrm{H}), 4.41(\mathrm{td}, J=12.5$, $5.4 \mathrm{~Hz}, 2 \mathrm{H}), 3.99-3.96(\mathrm{~m}, 1 \mathrm{H}), 3.54(\mathrm{t}, J=10.8 \mathrm{~Hz}, 1 \mathrm{H}) ;{ }^{13} \mathrm{C} \mathrm{NMR}\left(\mathrm{CDCl}_{3}, 150 \mathrm{MHz}\right) \delta 165.83,165.55$, 165.15, 164.86, 162.78, 162.62, 162.57, 136.22, 136.07, 131.93, 131.86, 128.63, 128.61, 128.17, 127.46, 127.41, 127.19, 122.30, 121.65, 121.59, 121.46, 114.52, 114.47, 114.43, 114.38, 73.69, 69.96, 69.90, 69.24, 67.23, 63.05; HRMS (ESI, $m / z):[\mathrm{M}+\mathrm{Na}]^{+}$, calcd for $\left[\mathrm{C}_{62} \mathrm{H}_{56} \mathrm{O}_{13} \mathrm{Na}\right]^{+}:$1027.3306; found 1027.3304.

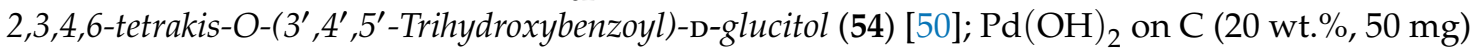
was added to a solution of compound $50(860 \mathrm{mg}, 0.46 \mathrm{mmol})$ in $20 \mathrm{~mL}$ of $\mathrm{MeOH}$ and $20 \mathrm{~mL}$ of THF under the argon. By the same procedure previously described for the preparation of compound 31, desired compound $54(321 \mathrm{mg}, 90 \%)$ was obtained as a yellow amorphous oil. $[\alpha]_{\mathrm{D}}^{20}=+58.0(\mathrm{c} 1.04$, $\mathrm{MeOH}) ;{ }^{1} \mathrm{H}$ NMR (Acetone $\left.-d_{6}, 600 \mathrm{MHz}\right) \delta 7.19(\mathrm{~s}, 2 \mathrm{H}), 7.06(\mathrm{~s}, 2 \mathrm{H}) \times 2,6.99(\mathrm{~s}, 2 \mathrm{H}), 5.81(\mathrm{t}, J=9.6 \mathrm{~Hz}$, $1 \mathrm{H}), 5.50(\mathrm{t}, J=9.8 \mathrm{~Hz}, 1 \mathrm{H}), 5.28-5.24(\mathrm{~m}, 1 \mathrm{H}), 4.49(\mathrm{dd}, J=12.4,2.1 \mathrm{~Hz}, 1 \mathrm{H}), 4.33-4.29(\mathrm{~m}, 2 \mathrm{H})$, $4.17-4.15(\mathrm{~m}, 1 \mathrm{H}), 3.72(\mathrm{t}, J=10.8 \mathrm{~Hz}, 1 \mathrm{H}) ;{ }^{13} \mathrm{C}$ NMR (Acetone $\left.-d_{6}, 150 \mathrm{MHz}\right) \delta 166.36,166.05,165.90$, $165.63,146.00,145.97,145.92,145.80,139.22,138.97,138.90,121.50,120.90,120.63,110.17,110.08,110.01$, 109.98, 77.57, 74.31, 70.74, 69.61, 67.44, 63.32; HRMS-ESI $(\mathrm{m} / \mathrm{z}):[\mathrm{M}+\mathrm{Na}]^{+}$, calcd for $\left[\mathrm{C}_{34} \mathrm{H}_{28} \mathrm{O}_{21} \mathrm{Na}\right]^{+}$: 795.1021; found 795.1019.

1,5-Anhydro-2,3,4,6-tetrakis-O-(3',4'-dihydroxybenzoyl)-D-glucitol (55); $\mathrm{Pd}(\mathrm{OH})_{2}$ on C (20 wt.\%, $50 \mathrm{mg}$ ) was added to a solution of compound 50 ( $365 \mathrm{mg}, 0.30 \mathrm{mmol})$ in $10 \mathrm{~mL}$ of $\mathrm{MeOH}$ and $10 \mathrm{~mL}$ of THF under the argon. By the same procedure previously described for the preparation of compound 31, desired compound $55(196 \mathrm{mg}, 92 \%)$ was obtained as a colorless amorphous oil. $[\alpha]_{\mathrm{D}}^{20}=+48.3$ (c $0.65, \mathrm{MeOH}) ;{ }^{1} \mathrm{H}$ NMR (Acetone $\left.-d_{6}, 600 \mathrm{MHz}\right) ; \delta 7.56(\mathrm{~d}, J=2.1 \mathrm{~Hz}, 1 \mathrm{H}), 7.49(\mathrm{dd}, J=8.4,1.9 \mathrm{~Hz}$, $1 \mathrm{H}), 7.43-7.33(\mathrm{~m}, 6 \mathrm{H}), 6.90(\mathrm{~d}, J=8.2 \mathrm{~Hz}, 1 \mathrm{H}), 6.84(\mathrm{dd}, J=11.3,8.2 \mathrm{~Hz}, 2 \mathrm{H}), 6.76(\mathrm{~d}, J=8.2 \mathrm{~Hz}, 1 \mathrm{H})$, $5.85(\mathrm{t}, J=9.6 \mathrm{~Hz}, 1 \mathrm{H}), 5.55(\mathrm{t}, J=9.8 \mathrm{~Hz}, 1 \mathrm{H}), 5.30(\mathrm{td}, J=10.1,5.4 \mathrm{~Hz}, 1 \mathrm{H}), 4.52(\mathrm{dd}, J=12.0,2.4 \mathrm{~Hz}$, $1 \mathrm{H}), 4.37-4.32(\mathrm{~m}, 2 \mathrm{H}), 4.20-4.17(\mathrm{~m}, 1 \mathrm{H}), 3.75(\mathrm{t}, J=10.8 \mathrm{~Hz}, 1 \mathrm{H}) ;{ }^{13} \mathrm{C}$ NMR (Acetone $-d_{6}, 150 \mathrm{MHz}$ ) $\delta$ 166.22, 166.00, 165.76, 165.56, 151.21, 151.01, 145.55, 145.39, 123.78, 123.71, 123.62, 122.53, 121.96, 121.74, 117.41, 117.29, 117.19, 117.09, 115.74, 115.68, 77.53, 74.40, 70.78, 69.87, 67.48, 63.52; HRMS (ES' $m / z):[\mathrm{M}-\mathrm{H}]^{-}$, calcd for $\left[\mathrm{C}_{34} \mathrm{H}_{27} \mathrm{O}_{17}\right]^{-}:$707.1248; found 707.1255.

1,5-Anhydro-2,3,4,6-tetrakis-O-(3,5-dihydroxybenzoyl)-D-glucitol (56); $\mathrm{Pd}(\mathrm{OH})_{2}$ on C (20 wt.\%, $\left.50 \mathrm{mg}\right)$ was added to a solution of compound 51 ( $365 \mathrm{mg}, 0.30 \mathrm{mmol}$ ) in $20 \mathrm{~mL}$ of $\mathrm{MeOH}$ and $20 \mathrm{~mL}$ of THF 
under the argon. By the same procedure previously described for the preparation of compound 31, desired compound 56 (210 mg, quant.) was obtained as a colorless amorphous oil. $[\alpha]_{\mathrm{D}}^{20}=+39.1$ (c 0.50 , $\mathrm{MeOH}) ;{ }^{1} \mathrm{H}$ NMR (Acetone $\left.-d_{6}, 600 \mathrm{MHz}\right) ; \delta 7.09(\mathrm{~s}, 2 \mathrm{H}), 6.95(\mathrm{~d}, J=1.4 \mathrm{~Hz}, 4 \mathrm{H}), 6.89(\mathrm{~d}, J=1.7 \mathrm{~Hz}$, $2 \mathrm{H}), 6.63-6.49(\mathrm{~m}, 4 \mathrm{H}), 5.90(\mathrm{t}, J=9.6 \mathrm{~Hz}, 1 \mathrm{H}), 5.59(\mathrm{t}, J=9.6 \mathrm{~Hz}, 1 \mathrm{H}), 5.37(\mathrm{td}, J=10.0,5.6 \mathrm{~Hz}, 1 \mathrm{H}), 4.55$ $(\mathrm{d}, J=12.4 \mathrm{~Hz}, 1 \mathrm{H}), 4.43(\mathrm{dd}, J=12.2,4.6 \mathrm{~Hz}, 1 \mathrm{H}), 4.37(\mathrm{dd}, J=11.3,5.5 \mathrm{~Hz}, 1 \mathrm{H}), 4.25(\mathrm{dd}, J=9.8,4.3 \mathrm{~Hz}$, $1 \mathrm{H}), 3.80(\mathrm{t}, J=10.8 \mathrm{~Hz}, 1 \mathrm{H}){ }^{13} \mathrm{C}$ NMR (Acetone $\left.-d_{6}, 150 \mathrm{MHz}\right) \delta 166.33,166.13,165.82,165.67,159.36$, 159.31, 159.24, 132.73, 132.10, 131.91, 129.67, 128.96, 108.81, 108.74, 108.65, 108.39, 108.24, 108.08, 77.25, 74.65, 70.85, 69.79, 67.25, 63.45; HRMS $\left(\mathrm{ES}^{-}, \mathrm{m} / \mathrm{z}\right):[\mathrm{M}-\mathrm{H}]^{-}$, calcd for $\left[\mathrm{C}_{34} \mathrm{H}_{27} \mathrm{O}_{17}\right]^{-}:$707.1248; found 707.1255.

1,5-Anhydro-2,3,4,6-tetrakis-O-(3-hydroxybenzoyl)-D-glucitol (57); $\mathrm{Pd}(\mathrm{OH})_{2}$ on C (20 wt.\%, $\left.50 \mathrm{mg}\right)$ was added to a solution of compound $52(300 \mathrm{mg}, 0.30 \mathrm{mmol})$ in $20 \mathrm{~mL}$ of MeOH and $20 \mathrm{~mL}$ of THF under the argon. By the same procedure previously described for the preparation of compound 31, desired compound $57(181 \mathrm{mg}, 94 \%)$ was obtained as a colorless amorphous oil. $[\alpha]_{\mathrm{D}}^{20}=+36.4(\mathrm{c} 0.70, \mathrm{MeOH}) ;{ }^{1} \mathrm{H}$ NMR (Acetone $\left.-d_{6}, 600 \mathrm{MHz}\right) ; \delta 7.55-6.97(\mathrm{~m}, 20 \mathrm{H}), 5.94(\mathrm{t}, J=9.5 \mathrm{~Hz}, 1 \mathrm{H}), 5.65(\mathrm{t}, J=9.6 \mathrm{~Hz}, 1 \mathrm{H}), 5.41$ $(\mathrm{td}, J=10.1,5.4 \mathrm{~Hz}, 1 \mathrm{H}), 4.57(\mathrm{dd}, J=12.2,2.6 \mathrm{~Hz}, 1 \mathrm{H}), 4.47(\mathrm{dd}, J=12.4,4.8 \mathrm{~Hz}, 1 \mathrm{H}), 4.39(\mathrm{dd}, J=11.2$, $5.7 \mathrm{~Hz}, 1 \mathrm{H}), 4.29-4.26(\mathrm{~m}, 1 \mathrm{H}), 3.83(\mathrm{t}, J=10.8 \mathrm{~Hz}, 1 \mathrm{H}) ;{ }^{13} \mathrm{C}$ NMR (Acetone $\left.-d_{6}, 150 \mathrm{MHz}\right) \delta 166.38$, 166.23, 165.90, 165.82, 158.46, 158.25, 132.14, 131.57, 131.43, 130.49, 130.44, 130.40, 129.72, 129.00, 126.08, $121.43,121.39,121.21,121.08,117.04,116.93,116.88,116.73,77.23,74.83,70.96,70.23,67.33,63.83$; HRMS $\left(\mathrm{ES}^{-}, \mathrm{m} / \mathrm{z}\right):[\mathrm{M}-\mathrm{H}]^{-}$, calcd for $\left[\mathrm{C}_{34} \mathrm{H}_{27} \mathrm{O}_{13}\right]^{-}:$: 643.1452; found 643.1459.

1,5-Anhydro-2,3,4,6-tetrakis-O-(4-hydroxybenzoyl)-D-glucitol (58); $\mathrm{Pd}(\mathrm{OH})_{2}$ on C (20 wt.\%, $\left.50 \mathrm{mg}\right)$ was added to a solution of compound $54(288 \mathrm{mg}, 0.29 \mathrm{mmol})$ in $15 \mathrm{~mL}$ of MeOH and $15 \mathrm{~mL}$ of THF under the argon. By the same procedure previously described for the preparation of compound 31, desired compound 58 (190 mg, quant.) was obtained as a colorless amorphous oil. $[\alpha]_{\mathrm{D}}^{20}=+45.3$ (c 0.50 , $\mathrm{MeOH}) ;{ }^{1} \mathrm{H}$ NMR (Acetone $\left.-d_{6}, 600 \mathrm{MHz}\right) ; \delta 7.93-7.76(\mathrm{~m}, 8 \mathrm{H}), 6.92-6.77(\mathrm{~m}, 8 \mathrm{H}), 5.89(\mathrm{t}, J=9.6 \mathrm{~Hz}$, $1 \mathrm{H}), 5.61(\mathrm{t}, J=9.8 \mathrm{~Hz}, 1 \mathrm{H}), 5.34(\mathrm{td}, J=10.0,5.3 \mathrm{~Hz}, 1 \mathrm{H}), 4.55(\mathrm{dd}, J=12.4,2.7 \mathrm{~Hz}, 1 \mathrm{H}), 4.41-4.35$ $(\mathrm{m}, 2 \mathrm{H}), 4.23-4.20(\mathrm{~m}, 1 \mathrm{H}), 3.77(\mathrm{t}, J=10.8 \mathrm{~Hz}, 1 \mathrm{H}) ;{ }^{13} \mathrm{C}$ NMR (Acetone $\left.-d_{6}, 150 \mathrm{MHz}\right) \delta 166.17$, $165.99,165.69,165.54,163.17,163.13,162.88,162.82,132.75,132.68,132.61,132.58,121.99,121.44,121.21$, $\left.121.19,116.09,116.05,116.00,115.94,77.45,74.47,70.76,70.05,67.50,63.65 ; \mathrm{HRMS}^{(E S}{ }^{-}, m / z\right):[\mathrm{M}-\mathrm{H}]^{-}$, calcd for $\left[\mathrm{C}_{34} \mathrm{H}_{27} \mathrm{O}_{13}\right]^{-}:$643.1452; found 643.1459 .

Benzyl 2,3,4,6-tetrakis-O-(3',4',5'-tribenzyloxybenzoyl)- $\alpha, \beta$-D-glucopyranoside (60); Compound 59 [43] (211 mg, $0.78 \mathrm{mmol})$, EDC.HCl (1.2 g, $6.3 \mathrm{mmol})$, compound 16 (2.06 g, $4.7 \mathrm{mmol})$, DMAP (47.7 mg, $0.39 \mathrm{mmol}$ ) in $30 \mathrm{~mL}$ of DCM was stirred at rt for overnight. After the addition $20 \mathrm{~mL}$ of water, the reaction mixture was extracted with DCM $(20 \mathrm{~mL})$. The combined organic layer was washed with water $(30 \mathrm{~mL} \times 4)$ and brine, dried over $\mathrm{Na}_{2} \mathrm{SO}_{4}$, filtered and concentrated under reduced pressure. The crude was purified by C.C $\left(\mathrm{CHCl}_{3} / \mathrm{MeOH}=200 / 1\right)$ to obtain desired compound $60(791 \mathrm{mg}, 52 \%)$ as a colorless amorphous oil. $[\alpha]_{\mathrm{D}}^{20}=+18.1$ (c 1.00, $\left.\mathrm{CHCl}_{3}\right) ;{ }^{1} \mathrm{H} \mathrm{NMR}\left(\mathrm{CDCl}_{3}, 600 \mathrm{MHz}\right.$ ) (assigned for the major anomer; $\alpha) \delta 7.42-7.16(\mathrm{~m}, 80 \mathrm{H}), 6.21(\mathrm{t}, J=10.0,1 \mathrm{H}), 5.69(\mathrm{t}, J=10.0 \mathrm{~Hz}, 1 \mathrm{H}), 5.45(\mathrm{~d}, J=3.8 \mathrm{~Hz}$, $1 \mathrm{H}), 5.12-4.95(\mathrm{~m}, 16 \mathrm{H}), 4.73(\mathrm{dd}, J=3.1,12.4 \mathrm{~Hz}, 1 \mathrm{H}), 4.54-4.51(\mathrm{~m}, 1 \mathrm{H}), 4.73(\mathrm{dd}, J=3.1,12.4 \mathrm{~Hz}, 1 \mathrm{H})$; ${ }^{13} \mathrm{C} \mathrm{NMR}\left(\mathrm{CDCl}_{3}, 150 \mathrm{MHz}\right)$ (assigned for the major anomer; $\left.\alpha\right) \delta 165.68,165.16,152.55,152.52,152.49$, 143.00, 142.86, 142.69, 142.64, 137.41, 137.36, 137.32, 136.56, 136.53, 136.48, 136.40, 136.29, 128.54, 128.46, $128.38,128.29,128.24,128.16,128.12,128.09,128.05,127.98,127.94,127.92,127.88,127.81,127.58,127.54$, 127.52, 124.64, 124.15, 124.02, 123.85, 109.23, 109.16, 109.01, 95.27, 75.11, 75.09, 75.06, 72.17, 71.18, 71.15, 71.05, 70.16, 69.96, 67.96, 63.17. HRMS (ESI, $m / z):[\mathrm{M}+\mathrm{Na}]^{+}$, calcd for $\left[\mathrm{C}_{125} \mathrm{H}_{106} \mathrm{O}_{22} \mathrm{Na}\right]^{+}:$1981.7073; found 1981.7067 .

2,3,4,6-tetrakis-O-(3', $4^{\prime}, 5^{\prime}$-Trihydroxybenzoyl)- $\alpha, \beta$-D-glucopyranose (61) [55]; $\mathrm{Pd}(\mathrm{OH})_{2}$ on C (20 wt.\%, $20 \mathrm{mg}$ ) was added to a solution of compound $54(100 \mathrm{mg}, 0.51 \mathrm{mmol})$ in $10 \mathrm{~mL}$ of $\mathrm{MeOH}$ and $10 \mathrm{~mL}$ of THF under the argon. By the same procedure previously described for the preparation of compound 31, desire compound $58(34 \mathrm{mg}, 85 \%)$ was obtained as a colorless amorphous oil. $[\alpha]_{\mathrm{D}}^{20}=+54.4(\mathrm{c} 0.30$, $\mathrm{MeOH}) ;{ }^{1} \mathrm{H}$ NMR $\left(\mathrm{CDCl}_{3}, 600 \mathrm{MHz}\right) \delta[\alpha$-form $7.20(\mathrm{~s}, 2 \mathrm{H}), 7.09(\mathrm{~s}, 2 \mathrm{H}), 7.07$ (s, 2H), 7.00 (s, $2 \mathrm{H}), 6.11$ $(\mathrm{t}, J=9.6 \mathrm{~Hz}, 1 \mathrm{H}), 5.62(\mathrm{~d}, J=3.4 \mathrm{~Hz}, 1 \mathrm{H}), 5.56(\mathrm{t}, J=10.2 \mathrm{~Hz}, 1 \mathrm{H}), 5.16(\mathrm{dd}, J=10.3,3.5 \mathrm{~Hz}, 1 \mathrm{H}), 4.47$ 
$(\mathrm{dd}, J=2.4,11.4 \mathrm{~Hz}, 1 \mathrm{H}), 4.35(\mathrm{dd}, J=12.6,4.8 \mathrm{~Hz}, 1 \mathrm{H}), 4.32-4.29(\mathrm{~m}, 1 \mathrm{H}) ;[\beta$-form] $7.19(\mathrm{~s}, 2 \mathrm{H}), 7.08$ $(\mathrm{s}, 2 \mathrm{H}), 7.05(\mathrm{~s}, 2 \mathrm{H}), 6.95(\mathrm{~s}, 2 \mathrm{H}), 5.80(\mathrm{t}, J=9.6 \mathrm{~Hz}, 1 \mathrm{H}), 5.51(\mathrm{t}, J=9.6 \mathrm{~Hz}, 1 \mathrm{H}), 5.29(\mathrm{t}, J=9.0 \mathrm{~Hz}, 1 \mathrm{H})$, $5.24(\mathrm{~d}, J=9.0 \mathrm{~Hz}, 1 \mathrm{H}), 4.50(\mathrm{~d}, J=1.7,11.4 \mathrm{~Hz}, 1 \mathrm{H}), 4.31(\mathrm{~m}, 2 \mathrm{H}) ;{ }^{13} \mathrm{C}$ NMR (Acetone $-d_{6}, 150 \mathrm{MHz}$ ) (assigned for the major anomer; $\alpha$ ) $\delta 166.48,166.22,165.99,165.73,146.04,145.99,145.84,139.27,139.24$, 139.00, 138.93, 121.61, 121.02, 120.78, 120.67, 110.21, 110.17, 110.06, 110.01, 91.04, 73.02, 70.70, 69.88, 68.55, 63.27; HRMS (ESI, $\mathrm{m} / \mathrm{z}):[\mathrm{M}+\mathrm{Na}]^{+}$, calcd for $\left[\mathrm{C}_{62} \mathrm{H}_{56} \mathrm{O}_{13} \mathrm{Na}\right]^{+}:$: 811.0970; found 811.0970.

1,5-Anhydro-2,3-bis-O-(3', 4' ,5'-tribenzyloxybenzoyl)-D-glucitol (62); Compound 21 (3.5 g, $3.2 \mathrm{mmol})$, iodine $(0.71 \mathrm{~g}, 5.6 \mathrm{mmol})$ in $100 \mathrm{~mL}$ of $\mathrm{DCM}$ and $50 \mathrm{~mL}$ of $\mathrm{MeOH}$ was stirred at $70{ }^{\circ} \mathrm{C}$ for 7 days. The reaction mixture was washed with sodium thiosulfate solution and brine. The crude product was purified by C.C (DCM/MeOH $=4 / 1)$ to obtain desired compound $62(3.0 \mathrm{~g}, 93 \%)$ as a white solid. $[\alpha]_{\mathrm{D}}^{20}=+80.3\left(\mathrm{c} 1.06, \mathrm{CHCl}_{3}\right) ;{ }^{1} \mathrm{H} \mathrm{NMR}\left(\mathrm{CDCl}_{3}, 600 \mathrm{MHz}\right) \delta 7.38-7.21(\mathrm{~m}, 32 \mathrm{H}), 5.34(\mathrm{t}, J=9.0 \mathrm{~Hz}, 1 \mathrm{H})$, 5.28-5.26 (m, 1H), 5.07-4.97 (m, 12H), 4.35-4.32 (m, 1H), 4.00-3.99 (m, 1H), 3.91-3.88 (m, 2H), 3.51-3.48 $(\mathrm{m}, 2 \mathrm{H}), 3.07(\mathrm{~d}, J=4.0 \mathrm{~Hz}, 1 \mathrm{H}), 2.01(\mathrm{t}, J=6.0 \mathrm{~Hz}, 1 \mathrm{H}) ;{ }^{13} \mathrm{C} \mathrm{NMR}\left(\mathrm{CDCl}_{3}, 150 \mathrm{MHz}\right) \delta 167.2,165.3$, 152.6, 143.1, 142.9, 137.3, 136.5, 136.4, 128.5, 128.4, 128.2, 128.1, 128.0, 127.7, 127.5, 109.3, 109.1, 80.4, 78.5, 75.1, 71.1, 67.0, 69.8, 66.9, 62.4; HRMS-ESI $(m / z):[\mathrm{M}+\mathrm{Na}]^{+}$, calcd for $\left[\mathrm{C}_{62} \mathrm{H}_{56} \mathrm{O}_{13} \mathrm{Na}\right]^{+}:$1031.3612; found 1031.3619.

1,5-Anhydro-2,3-bis-O-(3', 4', 5'-tribenzyloxybenzoyl)-4,6-bis-O-(3',5'-dimetoxymetoxy-4'-benzyloxybenzoyl) -D-glucitol (64); Compound 62 (2.5 g, $2.5 \mathrm{mmol}), 63$ [46] (2.5 g, $6.2 \mathrm{mmol})$, EDC.HCl (1.4 g, $7.2 \mathrm{mmol})$, DMAP $(0.15 \mathrm{~g}, 1.2 \mathrm{mmol})$ in $15 \mathrm{~mL}$ of DCM was stirred at $\mathrm{rt}$ for $18 \mathrm{~h}$. After the addition $30 \mathrm{~mL}$ of water, the reaction mixture was extracted with EtOAc $(3 \times 50 \mathrm{~mL})$. The combined organic layer was washed with brine, dried over $\mathrm{Na}_{2} \mathrm{SO}_{4}$, filtered and concentrated under reduced pressure. The crude was purified by C.C $\left(\mathrm{CHCl}_{3}\right.$ /acetone $\left.=100 / 1\right)$ to obtain $64(2.3 \mathrm{~g}, 55 \%$ yield $)$ as a colorless amorphous oil. $[\alpha]_{\mathrm{D}}^{20}=+24.7\left(\mathrm{c} 2.15\right.$ in $\left.\mathrm{CHCl}_{3}\right) ;{ }^{1} \mathrm{H}$ NMR $\left(\mathrm{CDCl}_{3}, 600 \mathrm{MHz}\right) \delta 7.58(\mathrm{~s}, 2 \mathrm{H}), 7.46-7.25(\mathrm{~m}, 50 \mathrm{H}), 5.85$ $(\mathrm{t}, J=10 \mathrm{~Hz}, 1 \mathrm{H}), 5.61(\mathrm{t}, J=10.0 \mathrm{~Hz}, 1 \mathrm{H}), 5.28-5.26(\mathrm{~m}, 1 \mathrm{H}), 5.22-4.93(\mathrm{~m}, 24 \mathrm{H}), 4.73-4.71(\mathrm{~m}, 1 \mathrm{H})$, $4.50-4.47(\mathrm{~m}, 1 \mathrm{H}), 4.35-4.32(\mathrm{~m}, 1 \mathrm{H}), 4.06-4.03(\mathrm{~m}, 1 \mathrm{H}), 3.56(\mathrm{t}, J=11.0 \mathrm{~Hz}, 1 \mathrm{H}), 3.48(\mathrm{~s}, 6 \mathrm{H}), 3.42(\mathrm{~s}, 6 \mathrm{H})$; ${ }^{13} \mathrm{C}$ NMR $\left(\mathrm{CDCl}_{3}, 150 \mathrm{MHz}\right) \delta 165.7,165.5,165.1,164.8,152.5,150.9,150.8,143.7,143.4,142.8,142.7$, 137.4, 137.3, 137.2, 136.5, 136.4, 132.4, 130.8, 128.7, 128.5, 128.4, 128.3, 128.2, 128.1, 128.0, 127.9, 127.8, 127.6, 127.5, 125.1, 124.2, 124.1, 124.0, 112.4, 112.3, 109.1, 109.0, 95.4, 75.2, 75.1, 75.0, 74.4, 71.1, 71.0, 70.6, 69.3, 68.1, 67.1, 63.3, 56.4; HRMS-ESI $(m / z)$ : $[\mathrm{M}+\mathrm{Na}]^{+}$, calcd for $\left[\mathrm{C}_{98} \mathrm{H}_{92} \mathrm{O}_{25} \mathrm{Na}\right]^{+}:$1691.5824; found 1691.5825 .

1,5-Anhydro-2,3-bis-O-(3', 4', 5' -tribenzyloxybenzoyl)-4,6-bis-O-(3', 5'-dihydroxy-4'-benzyloxybenzoyl) -D-glucitol (65); Compound $64(2.0 \mathrm{~g}, 1.2 \mathrm{mmol})$ in $8 \mathrm{~mL}$ of THF solution was added $8.8 \mathrm{~mL}$ of 2-propanol and $0.2 \mathrm{~mL}$ of conc. $\mathrm{HCl}$ solution, and the mixture was stirred at $60{ }^{\circ} \mathrm{C}$ for $6 \mathrm{~h}$. After the addition $3 \mathrm{~mL}$ of $\mathrm{NaHCO}_{3}$, the mixture was extracted with EtOAc $(3 \times 100 \mathrm{~mL})$. The combined organic layer was washed with brine, dried over $\mathrm{Na}_{2} \mathrm{SO}_{4}$, filtered and concentrated under reduced pressure. The crude was purified by C.C $\left(\mathrm{CHCl}_{3} / \mathrm{MeOH}=1000 / 1-500 / 1\right)$ to obtain $65(1.7 \mathrm{~g}, 93 \%$ yield $)$ as a white amorphous oil. $[\alpha]_{\mathrm{D}}^{20}=+26.8\left(\mathrm{c} 2.36, \mathrm{CHCl}_{3}\right) ;{ }^{1} \mathrm{H} \mathrm{NMR}\left(\mathrm{CDCl}_{3}, 600 \mathrm{MHz}\right) \delta 7.43-7.14(\mathrm{~m}, 48 \mathrm{H}), 6.00$ $(\mathrm{s}, 4 \mathrm{H}), 5.82(\mathrm{t}, J=9.5 \mathrm{~Hz}, 1 \mathrm{H}), 5.59(\mathrm{t}, J=9.5 \mathrm{~Hz}, 1 \mathrm{H}), 5.37-5.30(\mathrm{~m}, 1 \mathrm{H}), 5.10-4.82(\mathrm{~m}, 16 \mathrm{H}), 4.70-4.68$ $(\mathrm{m}, 1 \mathrm{H}), 4.54-4.52(\mathrm{~m}, 1 \mathrm{H}), 4.45-4.42(\mathrm{~m}, 1 \mathrm{H}), 3.97-3.95(\mathrm{~m}, 1 \mathrm{H}), 3.57(\mathrm{t}, J=10.5 \mathrm{~Hz}, 1 \mathrm{H}) ;{ }^{13} \mathrm{C}$ NMR $\left(\mathrm{CDCl}_{3}, 150 \mathrm{MHz}\right) \delta 166.2,165.8,165.5,165.2,152.5,149.0,148.9,142.8,142.7,138.2,137.8,137.3,137.2$, 136.6, 136.4, 136.2,128.8, 128.7, 128.5, 128.4, 128.3, 128.1, 128.0, 127.9, 127.7, 127.5, 124.5, 123.9, 123.8, 109.9, 109.8, 109.1, 108.9, 77.6, 75.2, 75.1, 75.0, 74.3, 71.1, 71.0, 70.7, 69.7, 68.6, 67.3, 62.6; HRMS-ESI $(\mathrm{m} / \mathrm{z})$ : $[\mathrm{M}+\mathrm{Na}]^{+}$, calcd for $\left[\mathrm{C}_{90} \mathrm{H}_{76} \mathrm{O}_{21} \mathrm{Na}\right]^{+}:$1515.4775; found 1515.4777.

Benzyl protected 1,5-AG-tellimagrandin analog (66); To a solution of $\mathrm{CuCl}_{2}$ (135 $\left.\mathrm{mg}, 1.0 \mathrm{mmol}\right)$ in $10 \mathrm{~mL}$ of $\mathrm{MeOH}$ was added $n$-butylamine $(400 \mu \mathrm{L}, 4.0 \mathrm{mmol})$. After the stirred at $\mathrm{rt}$ for $1.5 \mathrm{~h}$, the mixture was added to solution of compound 65 (500 mg, $0.34 \mathrm{mmol}$ ) in $20 \mathrm{~mL}$ of 1,2-dichloroethane (DCE) and stirred at $\mathrm{rt}$ for $30 \mathrm{~min}$. The reaction mixture was diluted with $50 \mathrm{~mL}$ of diethyl ether, and $50 \mathrm{~mL}$ of $5 \mathrm{M}$ aq. $\mathrm{HCl}$ and $50 \mathrm{~mL}$ of diethyl ether were added. The separating organic layer washed with water, $\mathrm{NaHCO}_{3}$ and brine. The organic layer was dried over $\mathrm{Na}_{2} \mathrm{SO}_{4}$, filtered and concentrated under reduced pressure. The crude was purified by C.C $(\mathrm{DCM} / \mathrm{MeOH}=250 / 1)$ and HPLC (column, 
FNED01048, $250 \times 20 \mathrm{~mm}$, SG80-5 $\mu \mathrm{m}$, eluant DCM/MeOH = 200/1) to afford $66(237 \mathrm{mg}, 48 \%)$ as a peal yellow amorphous oil. $[\alpha]_{\mathrm{D}}^{20}=+69.5$ (c 1.43, $\left.\mathrm{CHCl}_{3}\right) ;{ }^{1} \mathrm{H} \mathrm{NMR}\left(\mathrm{CDCl}_{3}, 600 \mathrm{MHz}\right) \delta 7.43-7.23$ $(\mathrm{m}, 50 \mathrm{H}), 6.75(\mathrm{~s}, 1 \mathrm{H}), 6.67(\mathrm{~s}, 1 \mathrm{H}), 5.67(\mathrm{t}, J=10.0 \mathrm{~Hz}, 1 \mathrm{H}), 5.37-5.34(\mathrm{~m}, 1 \mathrm{H}), 5.29(\mathrm{t}, J=10.0 \mathrm{~Hz}, 1 \mathrm{H})$, 5.25-5.24 (m, 1H), 5.13-4.85 (m, 16H), 4.47-4.45 (m, 1H), $3.98(\mathrm{~d}, J=12.5 \mathrm{~Hz}, 1 \mathrm{H}), 3.96-3.94(\mathrm{~m} .1 \mathrm{H})$, $3.46(\mathrm{t}, J=11.0 \mathrm{~Hz}, 1 \mathrm{H}) ;{ }^{13} \mathrm{C} \mathrm{NMR}\left(\mathrm{CDCl}_{3}, 150 \mathrm{MHz}\right) \delta 167.1,166.6,165.8,165.2,152.5,149.0,147.0$, $142.8,137.4,137.3,136.5,136.4,136.3,136.2,135.7,135.5,130.3,129.6,129.0,128.9,128.6,128.5,128.4$, 128.3, 128.1, 128.0, 127.9, 127.8, 127.6, 127.5, 123.9, 113.7, 113.2, 109.2, 108.4, 107.9, 75.6, 75.1, 75.0, 74.4, 71.1, 71.0, 70.8, 70.1, 67.7, 63.6; HRMS-ESI $(m / z):[\mathrm{M}+\mathrm{Na}]^{+}$, calcd for $\left[\mathrm{C}_{90} \mathrm{H}_{74} \mathrm{O}_{21} \mathrm{Na}\right]^{+}: 1513.4619$; found 1513.4620 .

1,5-Anhydro-2,3-bis-O-(3', ,4',5'-trihydroxybenzoyl)-4,6-O-hexahydroxydiphenyl-D-glucitol (67); $\mathrm{Pd}(\mathrm{OH})_{2}$ on C (20 wt.\%, $20 \mathrm{mg}$ ) was added to a solution of compound 66 (60 mg, $0.40 \mathrm{mmol})$ in $2 \mathrm{~mL}$ of $\mathrm{MeOH}$ and $2 \mathrm{~mL}$ of THF under the argon. By the same procedure previously described for the preparation of compound procedure described for previously 31 preparation, desired compound 67 (32 mg, quant.) was obtained as a peal yellow amorphous oil. $[\alpha]_{\mathrm{D}}^{20}=+113.3$ (c $\left.0.29, \mathrm{MeOH}\right) ;{ }^{1} \mathrm{H} \mathrm{NMR}$ $\left(\mathrm{CD}_{3} \mathrm{OD}, 600 \mathrm{MHz}\right) \delta 7.00(\mathrm{~s}, 2 \mathrm{H}), 6.95(\mathrm{~s}, 2 \mathrm{H}), 6.60(\mathrm{~s}, 1 \mathrm{H}), 6.40(\mathrm{~s}, 1 \mathrm{H}), 5.61(\mathrm{t}, J=9.5 \mathrm{~Hz}, 1 \mathrm{H}), 5.27-5.19$ $(\mathrm{m}, 2 \mathrm{H}), 5.03(\mathrm{t}, J=10.0 \mathrm{~Hz}, 1 \mathrm{H}), 4.25-4.22(\mathrm{~m}, 1 \mathrm{H}), 4.08-4.07(\mathrm{~m}, 1 \mathrm{H}), 3.80(\mathrm{t}, J=13.0 \mathrm{~Hz}, 1 \mathrm{H}), 3.54$ $(\mathrm{t}, J=11.0 \mathrm{~Hz}, 1 \mathrm{H}) ;{ }^{13} \mathrm{C} \mathrm{NMR}\left(\mathrm{CD}_{3} \mathrm{OD}, 150 \mathrm{MHz}\right) ; 171.1,170.7,168.8,168.3,150.6,147.6,147.1,144.6$, 143.5, 141.8, 139.7, 126.8, 126.7, 126.3, 120.7, 120.3, 120.1, 119.4, 111.1, 110.8, 108.3, 107.7, 78.7, 78.5, 76.2, 72.1, 72.0, 71.8, 71.4, 71.3, 69.4, 68.7, 64.7; HRMS (ESI, $m / z):[\mathrm{M}+\mathrm{Na}]^{+}$, calcd for $\left[\mathrm{C}_{34} \mathrm{H}_{26} \mathrm{O}_{21} \mathrm{Na}^{+}\right.$: 793.0864; found 793.0865 .

Supplementary Materials: The following are available online ${ }^{1} \mathrm{H}$ NMR, ${ }^{13} \mathrm{C}$ NMR spectra of compounds 31-44, $54-58,61$ and 67.

Author Contributions: Experiment: S.M. (Shota Machida), S.M. (Saki Mukai), R.K. and M.F.; Writing-Original Draft preparation: S.M. (Shota Machida); Writing-Review and Editing: S.M. (Shota Machida), H.S. and T.U.; project administration: T.U.

Funding: This study was partly supported by Japan Society for the Promotion of Science (JSPS), 18K06725 (T.U.) and Nihon University Mutidisciplinary Research Grant for 2019 (19-016).

Acknowledgments: We thank Koichi Metori (Chemical Analysis Center, School of Pharmacy, Nihon University) for performing mass measurements.

Conflicts of Interest: The authors declare no conflict of interest.

\section{References}

1. Oizumi, T.; Daimon, M.; Jimbu, Y.; Wada, K.; Kameda, W.; Susa, S.; Yamaguchi, H.; Ohnuma, H.; Tominaga, M.; Kato, T. Impaired Glucose Tolerance is a Risk Factor for Stroke in a Japanese Sample-the Funagata Study. Metabolism 2008, 57, 333-338. [CrossRef] [PubMed]

2. Bornfeldt, K.E.; Tabas, I. Insulin Resistance, Hyperglycemia, and Atherosclerosis. Cell Metab. 2011, 14, 575-585. [CrossRef] [PubMed]

3. Ceriello, A.; Bortolotti, N.; Motz, E.; Pieri, C.; Marra, M.; Tonutti, L.; Lizzio, S.; Feletto, F.; Catone, B.; Taboga, C. Meal-Induced Oxidative Stress and Low-Density Lipoprotein Oxidation in Diabetes: The Possible Role of Hyperglycemia. Metabolism 1999, 48, 1503-1508. [CrossRef]

4. Ten Bruggencate, S.J.; Frederiksen, P.D.; Pedersen, S.M.; Floris-Vollenbroek, E.G.; Lucas-van de Bos, E.; van Hoffen, E.; Wejses, P.L. Dietary Milk-Fat-Globule Membrane Affects Resistance to Diarrheagenic Escherichia Coli in Healthy Adults in a Randomized, Placebo-Controlled, Double-Blind Study. J. Nutr. 2016, 146, $249-255$. [CrossRef] [PubMed]

5. Ceriello, A.; Genovese, S. Atherogenicity of Postprandial Hyperglycemia and Lipotoxicity. Rev. Endocr. Metab. Dis. 2016, 17, 111-116. [CrossRef] [PubMed]

6. Mah, E.; Bruno, R.S. Postprandial Hyperglycemia on Vascular Endothelial Function: Mechanisms and Consequences. Nutr. Res. 2012, 32, 727-740. [CrossRef] [PubMed]

7. Kitasato, L.; Tojo, T.; Hatakeyama, Y.; Kameda, R.; Hashikata, T.; Yamaoka-Tojo, M. Postprandial Hyperglycemia and Endothelial Function in Type 2 Diabetes: Focus on Mitiglinide. Cardiovasc. Diabetol. 2012, 11, 79. [CrossRef] 
8. Derosa, G.; Maffioli, P. $\alpha$-Glucosidase Inhibitors and their Use in Clinical Practice. Arch. Med. Sci. 2012, 8, 899-906. [CrossRef]

9. Tuccori, M.; Convertino, I.; Galiulo Maria, T.; Marino, A.; Capogrosso-Sansone, A.; Blandizzi, C. Diabetes Drugs and the Incidence of Solid Cancers: A Survey of the Current Evidence. Expert Opin. Drug Saf. 2017, 16, 1133-1148. [CrossRef]

10. Liu, Z.; Ma, S. Recent Advances in Synthetic $\alpha$-Glucosidase Inhibitors. Chem. Med. Chem. 2017, 12, 819-829. [CrossRef]

11. Singla, R.K.; Singh, R.; Dubey, A.K. Important Aspects of Post-Prandial Antidiabetic Drug, Acarbose. Curr. Top Med. Chem. 2016, 16, 2625-2633. [CrossRef] [PubMed]

12. Newman, D.J.; Cragg, G.M. Natural Products as Sources of New Drugs from 1981 to 2014. J. Nat. Prod. 2016, 79, 629-661. [CrossRef] [PubMed]

13. Che, C.-T.; Zhang, H. Plant natural products for human health. Int. J. Mol. Sci. 2019, 20, 830. [CrossRef] [PubMed]

14. Wright, G.D. Opportunities for Natural Products in 21(st) Century Antibiotic Discovery. Nat. Prod. Rep. 2017, 34, 694-701. [CrossRef]

15. Zhao, C.; Yang, C.F.; Wai, S.T.C.; Zhang, Y.B.; Portillo, M.P.; Paoli, P.; Wu, Y.; Cheang, W.S.; Liu, B.; Carpéné, C.; et al. Regulation of Glucose Metabolism by Bioactive Phytochemicals for the Management of Type 2 Diabetes Mellitus. Crit. Rev. Food Sci. 2019, 59, 830-847. [CrossRef]

16. Bi, W.; Gao, Y.; Shen, J.; He, C.; Liu, H.; Peng, Y.; Zhang, C.; Xiao, P. Traditional Uses, Phytochemistry, and Pharmacology of the Genus Acer (Maple): A review. J. Ethnopharmacol. 2016, 189, 31-60. [CrossRef]

17. González-Sarrías, A.; Li, L.; Seeram, N.P. Effects of Maple (Acer) Plant Part Extracts on Proliferation, Apoptosis and Cell Cycle Arrest of Human Tumorigenic and Non-Tumorigenic Colon Cells. Phyther. Res. 2012, 26, 995-1002. [CrossRef]

18. Bi, W.; Liu, H.; Shen, J.; Zhang, L.H.; Li, P.; Peng, B.; Cao, L.; Zhang, P.; He, C.; Xiao, P. Chemopreventive Effects of Ku-Jin Tea Against AOM-Induced Precancerous Colorectal Lesions in Rats and Metabolomic Analysis. Sci. Rep. 2017, 7, 15893. [CrossRef]

19. Han, S.S.; Lo, S.C.; Choi, Y.W.; Kim, J.H.; Baek, S.H. Antioxidant Activity of Crude Extract and Pure Compounds of Acer Ginnala Max. Bull. Korean Chem. Soc. 2004, 25, 389-391.

20. Royer, M.; Diouf, P.N.; Stevanovic, T. Polyphenol Contents and Radical Scavenging Capacities of Red Maple (Acer Rubrum L.) Extracts. Food Chem. Toxicol. 2011, 49, 2180-2188. [CrossRef]

21. Watanabe, M.; Prasad, D.H. Antioxidant Phenolic Constituents from the Leaves of Acer Ginnala var Aidzuense. J. Nat. Remedies 2017, 17, 9-12. [CrossRef]

22. Bi, W.; Shen, J.; Gao, Y.; He, C.; Peng, Y.; Xiao, P. Ku-Jin Tea (Acer Tataricum Subsp. Ginnala or A. Tataricum Subsp. Theiferum), an Underestimated Functional Beverage Rich in Antioxidant Phenolics. J. Funct. Foods 2016, 24, 75-84. [CrossRef]

23. Apostolidis, E.; Li, L.; Lee, C.; Seeram, N.P. In Vitro Evaluation of Phenolic-Enriched Maple Syrup Extracts for Inhibition of Carbohydrate Hydrolyzing Enzymes Relevant to Type 2 Diabetes Management. J. Funct. Foods 2011, 3, 100-106. [CrossRef]

24. Honma, A.; Koyama, T.; Yazawa, K. Antihyperglycemic effects of Japanese maple Acer amoenum leaf extract and its constituent corilagin. J. Wood Sci. 2010, 56, 507-512. [CrossRef]

25. Honma, A.; Koyama, T.; Yazawa, K. Anti-Hyperglycemic Effects of Sugar Maple Acer Saccharum and its Constituent Acertannin. Food Chem. 2010, 123, 390-394. [CrossRef]

26. Bock, K.; LaCour, N.F.; Jensen, S.R.; Nielsen, B.J. The structure of acertannin. Phytochemistry 1980, 19, 2033. [CrossRef]

27. Bate-Smith, E.C. Astringent Tannins of Acer Species. Phytochemistry 1977, 16, 1421-1426. [CrossRef]

28. Hatano, T.; Hattori, S.; Ikeda, Y.; Shingu, T.; Okuda, T. Tannins of Aceraceous Plants. Part II. Gallotannins Having a 1,5-Anhydro-D-Glucitol Core and Some Ellagitannins from Acer Species. Chem. Pharm. Bull. 1990, 38, 1902-1905. [CrossRef]

29. Wan, C.; Yuan, T.; Li, L.; Kandhi, V.; Cech, N.B.; Xie, M.; Seeram, N.P. Maplexins, New $\alpha$-Glucosidase Inhibitors from Red Maple (Acer Rubrum) Stems. Bioorganic Med. Chem. Lett. 2012, 22, 597-600. [CrossRef]

30. Yuan, T.; Wan, C.; Liu, K.; Seeram, N.P. New Maplexins F-I and Phenolic Glycosides from Red Maple (Acer Rubrum) Bark. Tetrahedron 2012, 68, 959-964. [CrossRef] 
31. Honma, A.; Koyama, T.; Yazawa, K. Anti-Hyperglycaemic Effects of the Japanese Red Maple Acer Pycnanthum and its Constituents the Ginnalins B and C. J. Enzyme Inhib. Med. Chem. 2011, 26, 176-180.

32. Ma, H.; Wang, L.; Niesen, D.B.; Cai, A.; Cho, B.P.; Tan, W.; Gu, Q.; Xu, J.; Seeram, N.P. Structure Activity Related, Mechanistic, and Modeling Studies of Gallotannins Containing a Glucitol-Core and $\alpha$-Glucosidase. RSC Adv. 2015, 5, 107904-107915. [CrossRef] [PubMed]

33. González-Sarrías, A.; Yuan, T.; Seeram, N.P. Cytotoxicity and Structure Activity Relationship Studies of Maplexins A-I, Gallotannins from Red Maple (Acer Rubrum). Food Chem. Toxicol. 2012, 50, 1369-1376. [CrossRef] [PubMed]

34. González-Sarrías, A.; Ma, H.; Edmonds, M.E.; Seeram, N.P. Maple Polyphenols, Ginnalins A-C, Induce Sand G2/M-Cell Cycle Arrest in Colon and Breast Cancer Cells Mediated by Decreasing Cyclins A and D1 Levels. Food Chem. 2013, 136, 636-642. [CrossRef] [PubMed]

35. Kamori, A.; Kato, A.; Miyawaki, S.; Koyama, J.; Nash, R.J.; Fleet, G.W.J.; Miura, D.; Ishikawa, F.; Adachi, I. Dual Action of Acertannins as Potential Regulators of Intracellular Ceramide Levels. Tetrahedron: Asymmetry 2016, 27, 1177-1185. [CrossRef]

36. Kato, A.; Koyama, J.; Shinzawa, K.; Imaeda, S.; Adachi, I.; Nash, R.J.; Fleet, G.W.J.; Shintani, M.; Takeuchi, C.; Ishikawa, F. Ginnalin B Induces Differentiation Markers and Modulates the Proliferation/Differentiation Balance via the Upregulation of NOTCH1 in Human Epidermal Keratinocytes. Bioorganic Med. Chem. 2019, 27, 2172-2180. [CrossRef]

37. Yokozawa, T.; Chen, C.P.; Dong, E.; Tanaka, T.; Nonaka, G.; Nishioka, I. Study on the Inhibitory Effect of Tannins and Flavonoids Against the 1,1-Diphenyl-2-Picrylhydrazyl Radical. Biochem. Pharmacol. 1998, 56, 213-222. [CrossRef]

38. Lee, D.Y.; Kim, H.W.; Yang, H.; Sung, S.H. Hydrolyzable Tannins from the Fruits of Terminalia Chebula Retz and their $\alpha$-Glucosidase Inhibitory Activities. Phytochemistry 2017, 137, 109-116. [CrossRef]

39. Wilkins, C.K.; Bohm, B.A. Ellagitannins from Tellima Grandiflora. Phytochemistry 1976, 15, 211-214. [CrossRef]

40. Zheng, S.; Laraia, L.; O'Connor, C.J.; Sorrell, D.; Tan, Y.S.; Xu, Z.; Venkitaraman, A.R.; Wu, W.; Spring, D.R. Synthesis and Biological Profiling of Tellimagrandin I and Analogues Reveals that the Medium Ring Can Significantly Modulate Biological Activity. Org. Biomol. Chem. 2012, 10, 2590-2593. [CrossRef]

41. Uchiyama, T.; Shishikura, K.; Ogawa, K.; Ohshima, Y.; Miyairi, S. An Efficient Method for the Preparation of 1,5-Anhydroalditol from Unprotected Carbohydrates via Glycopyranosyl Iodide. Tetrahedron Lett. 2016, 57, 5294-5296. [CrossRef]

42. Nie, X.; Wang, G. Synthesis of a Ring-Oxygenated Variant of the 2-Carboxy-6-Hydroxyoctahydroindole Core of Aeruginosin 298-A from Glucose. J. Org. Chem. 2005, 70, 8687-8692. [CrossRef] [PubMed]

43. Lonnecker, A.T.; Lim, Y.H.; Felder, S.E.; Besset, C.J.; Wooley, K.L. Four Different Regioisomeric Polycarbonates Derived from One Natural Product, D-Glucose. Macromolecules 2016, 49, 7857-7867. [CrossRef]

44. Tanaka, N.; Ogawa, I.; Yoshigase, S.; Nokami, J. Regioselective Ring Opening of Benzylidene Acetal Protecting Group(s) of Hexopyranoside Derivatives by DIBAL-H. Carbohydr. Res. 2008, 343, 2675-2679. [CrossRef] [PubMed]

45. Daragics, K.; Fügedi, P. Regio- and Chemoselective Reductive Cleavage of 4,6-O-Benzylidene-Type Acetals of Hexopyranosides Using $\mathrm{BH}_{3}$.THF-TMSOTf. Tetrahedron Lett. 2009, 50, 2914-2916. [CrossRef]

46. Haslam, E.; Radford, T. Synthesis of 1,3-Anhydro-D-Glucitol and Some Derivatives of 1,5-Anhydro-D-Glucitol. Carbohydr. Res. 1966, 2, 301-314. [CrossRef]

47. Qian, P.; Yao, W.; Huang, L.; Meng, X.; Li, Z. A Mild and Efficient Method for the Selective Cleavage of Primary p-Methoxybenzyl Protecting Group of Saccharides by $\mathrm{Co}_{2}(\mathrm{CO})_{8}-\mathrm{Me}_{2} \mathrm{PhSiH}-\mathrm{CO}$ System. Tetrahedron Lett. 2015, 56, 5238-5241. [CrossRef]

48. DeNinno, M.P.; Etienne, J.B.; Duplantier, K.C. A Method for the Selective Reduction of Carbohydrate 4,6-O-Benzylidene Acetals. Tetrahedron Lett. 1995, 36, 669-672. [CrossRef]

49. Cocinero, E.J.; Gamblin, D.P.; Davis, B.G.; Simons, J.P. The Building Blocks of Cellulose: The Intrinsic Conformational Structures of Cellobiose, its Epimer, Lactose, and their Singly Hydrated Complexes. J. Am. Chem. Soc. 2009, 131, 11117-11123. [CrossRef]

50. Ren, Y.; Himmeldirk, K.; Chen, X. Synthesis and Structure-Activity Relationship Study of Antidiabetic Penta-O-galloyl-D-glucopyranose and its Analogues. J. Med. Chem. 2006, 49, 2829-2837. [CrossRef] 
51. Tranchimand, S.; Tron, T.; Gaudin, C.; Iacazio, G. First Chemical Synthesis of Three Natural Depsides Involved in Flavonol Catabolism and Related to Quercetinase Catalysis. Synth. Commun. 2006, 36, 587-597. [CrossRef]

52. Fergusson, K.M.; Hird, M. The Dramatic Influence of the Location of Bend and of Lateral Fluoro Substitution on the Mesomorphic Properties of Angular Chiral Esters Based on a 1,3-Disubstituted Benzene Ring. J. Mater. Chem. 2010, 20, 3069-3078. [CrossRef]

53. Hawker, C.J.; Lee, R.; Frechet, J.M.J. One-Step Synthesis of Hyperbranched Dendritic Polyesters. J. Am. Chem. Soc. 1991, 113, 4583-4588. [CrossRef]

54. Furukawa, M.; Kamo, S.; Makino, M.; Kurita, M.; Tabata, K.; Matsuzaki, K.; Suzuki, T.; Uchiyama, T. Triterpenoid Glycosides from Ladenbergia Hexandra Klotzsch. Phytochemistry 2017, 136, 147-155. [CrossRef] [PubMed]

55. Gramshaw, J.W.; Haslam, E.; Haworth, R.D.; Searle, T. 571. Gallotannins. Part, V. The Structure of Penta- and Tetra-O-Galloylglucoses, and Some Observations on the Molecular Weights of the Gallotannins. J. Chem. Soc. 1962, 2944. [CrossRef]

56. Feldman, K.S.; Ensel, S.M.; Minard, R.D. Ellagitannin Chemistry. The First Total Chemical Synthesis of an Ellagitannin Natural Product, Tellimagrandin I. J. Am. Chem. Soc. 1994, 116, 1742-1745. [CrossRef]

57. Michihata, N.; Kaneko, Y.; Kasai, Y.; Tanigawa, K.; Hirokane, T.; Higasa, S.; Yamada, H. High-Yield Total Synthesis of (-)-Strictinin through Intramolecular Coupling of Gallates. J. Org. Chem. 2013, 78, 4319-4328. [CrossRef]

58. Yamada, H.; Nagao, K.; Dokei, K.; Kasai, Y.; Michihata, N. Total Synthesis of (-)-Corilagin. J. Am. Chem. Soc. 2008, 130, 7566-7567. [CrossRef]

59. Kasai, Y.; Michihata, N.; Nishimura, H.; Hirokane, T.; Yamada, H. Total Synthesis of (+)-Davidiin. Angew. Chemie. Int. Ed. 2012, 51, 8026-8029. [CrossRef]

60. Asakura, N.; Fujimoto, S.; Michihata, N.; Nishii, K.; Imagawa, H.; Yamada, H. Synthesis of Chiral and Modifiable Hexahydroxydiphenoyl Compounds. J. Org. Chem. 2011, 76, 9711-9719. [CrossRef]

61. Oki, T.; Masuda, M.; Furuta, S.; Nishiba, Y.; Suda, I. Radical Scavenging Activity of Fried Chips Made from Purple-Fleshed Sweet Potato. J. Jpn. Soc. Food Sci. 2011, 48, 926-932. [CrossRef]

62. Toda, M.; Kawabata, J.; Kasai, T. $\alpha$-Glucosidase Inhibitors from Clove (Syzgium aromaticum). Biosci. Biotechnol. Biochem. 2000, 64, 294-298. [CrossRef] [PubMed]

Sample Availability: Samples of the compounds are not available from the authors.

(C) 2019 by the authors. Licensee MDPI, Basel, Switzerland. This article is an open access article distributed under the terms and conditions of the Creative Commons Attribution (CC BY) license (http://creativecommons.org/licenses/by/4.0/). 